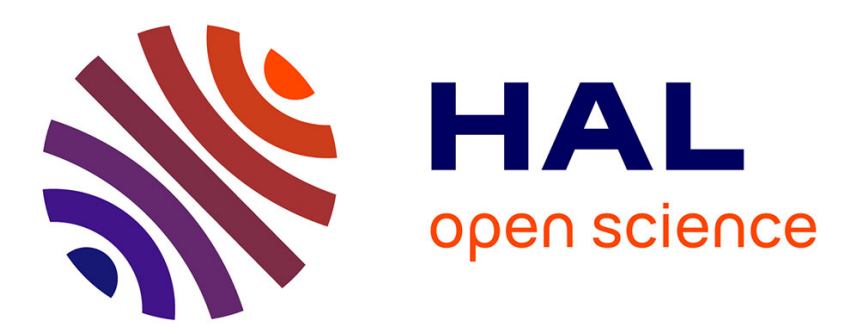

\title{
Apport de l'IRM multimodale et de la TEP-TDM à la 18F-FDOPA dans le suivi des tumeurs cérébrales irradiées
}

\author{
Alexandra Benarrous Sitbon
}

\section{- To cite this version:}

Alexandra Benarrous Sitbon. Apport de l'IRM multimodale et de la TEP-TDM à la 18F-FDOPA dans le suivi des tumeurs cérébrales irradiées. Médecine humaine et pathologie. 2015. dumas-01267055

\section{HAL Id: dumas-01267055 https://dumas.ccsd.cnrs.fr/dumas-01267055}

Submitted on 3 Feb 2016

HAL is a multi-disciplinary open access archive for the deposit and dissemination of scientific research documents, whether they are published or not. The documents may come from teaching and research institutions in France or abroad, or from public or private research centers.
L'archive ouverte pluridisciplinaire HAL, est destinée au dépôt et à la diffusion de documents scientifiques de niveau recherche, publiés ou non, émanant des établissements d'enseignement et de recherche français ou étrangers, des laboratoires publics ou privés. 
UNIVERSITE DE NICE SOPHIA ANTIPOLIS

UFR DES SCIENCES MEDICALES

\section{APPORT DE L'IRM MULTIMODALE ET DE LA TEP-TDM A LA ${ }^{18}$ F-FDOPA DANS LE SUIVI DES TUMEURS CEREBRALES IRRADIEES}

THESE D’EXERCICE DE MEDECINE

Présentée et soutenue publiquement

Le 8 septembre 2015

par

BENARROUS SITBON Alexandra

Née le 8 juin 1986 à Paris $19^{\text {ème }}$ (75019)

Interne des Hôpitaux

DIRECTEUR DE THESE :

Docteur Lydiane MONDOT

MEMBRES DU JURY :

Professeur Bernard PADOVANI

Président

Professeur Patrick CHEVALLIER

Assesseur

Professeur Jacques DARCOURT

Assesseur

Professeur Denys FONTAINE

Assesseur

Docteur Véronique BOURG

Assesseur

Docteur Fanny VANDENBOS

Assesseur 
UNIVERSITÉ DE NICE-SOPHIA ANTIPOLIS FACULTÉ DE MÉDECINE

Liste des professeurs au 1er décembre 2014 à la Faculté de Médecine de Nice

Doyen

Vice-Doyen

Assesseurs

Conservateur de la bibliothèque

Directrice administrative des services

Doyens Honoraires

\section{Professeurs Honoraires}

M. BALAS Daniel

M. BLAIVE Bruno

M. BOQUET Patrice

M. BOURGEON André

M. BOUTTÉ Patrick

M. BRUNETON Jean-Noël

Mme BUSSIERE Françoise

M. CAMOUS Jean-Pierre

M. CHATEL Marcel

M. COUSSEMENT Alain

M. DARCOURT Guy

M. DELLAMONICA Pierre

M. DELMONT Jean

M. DEMARD François

M. DOLISI Claude

$M$. FRANCO Alain

M. FREYCHET Pierre

M. GÉRARD Jean-Pierre

M. GILLET Jean-Yves

M. GRELLIER Patrick

M. HARTER Michel
M. BAQUÉ Patrick

M. BOILEAU Pascal

M. ESNAULT Vincent

M. CARLES Michel

Mme BREUIL Véronique

M. MARTY Pierre

Mme DE LEMOS Annelyse

Mme CALLEA Isabelle

M. AYRAUD Noël

M. RAMPAL Patrick

M. BENCHIMOL Daniel

M. INGLESAKIS Jean-André $M$. LALANNE Claude-Michel $\mathrm{M}$. LAMBERT Jean-Claude

M. LAZDUNSKI Michel

M. LEFEBVRE Jean-Claude

M. LE BAS Pierre

M. LE FICHOUX Yves

M. LOUBIERE Robert

M. MARIANI Roger

M. MASSEYEFF René

M.MATTEI Mathieu

M. MOUIEL Jean

Mme MYQUEL Martine

M. OLLIER Amédée

M. ORTONNE Jean-Paul

M. SCHNEIDER Maurice

M. SERRES Jean-Jacques

M. TOUBOL Jacques

M. TRAN Dinh Khiem

M. ZIEGLER Gérard 
M.C.A. Honoraire

M.C.U. Honoraires
Mlle ALLINE Madeleine

\author{
M. ARNOLD Jacques \\ M. BASTERIS Bernard \\ Mlle CHICHMANIAN Rose-Marie \\ Mme DONZEAU Michèle \\ M. EMILIOZZI Roméo \\ M. FRANKEN Philippe M. \\ GASTAUD Marcel \\ M.GIRARD-PIPAU Fernand \\ M. GIUDICELLI Jean \\ M. MAGNÉ Jacques \\ Mme MEMRAN Nadine \\ M. MENGUAL Raymond \\ M. POIRÉE Jean-Claude \\ Mme ROURE Marie-Claire
}

\section{PROFESSEURS CLASSE EXCEPTIONNELLE}

$\begin{array}{ll}\text { M. } & \text { AMIEL Jean } \\ \text { M. } & \text { BENCHIMOL Daniel } \\ \text { M. } & \text { BOILEAU Pascal } \\ \text { M. } & \text { DARCOURT Jacques } \\ \text { M. } & \text { DESNUELLE Claude } \\ \text { Mme } & \text { EULLER-ZIEGLER Liana } \\ \text { M. } & \text { FENICHEL Patrick } \\ \text { M. } & \text { FUZIBET Jean-Gabriel } \\ \text { M. } & \text { GASTAUD Pierre } \\ \text { M. } & \text { GILSON Éric } \\ \text { M. } & \text { GRIMAUD Dominique } \\ \text { M. } & \text { HASSEN KHODJA Reda } \\ \text { M. } & \text { HÉBUTERNE Xavier } \\ \text { M. } & \text { HOFMAN Paul } \\ \text { M. } & \text { LACOUR Jean-Philippe } \\ \text { Mme } & \text { LEBRETON Élisabeth } \\ \text { M. } & \text { MICHIELS Jean-François } \\ \text { M. } & \text { MOUROUX Jérôme } \\ \text { M. } & \text { PAQUIS Philippe } \\ \text { M. } & \text { PRINGUEY Dominique } \\ \text { M. } & \text { QUATREHOMME Gérald } \\ \text { M. } & \text { M.ROBERT Philippe } \\ \text { M. } & \text { SANTINI Joseph } \\ \text { M. } & \text { THYSS Antoine } \\ \text { M. } & \text { VAN OBBERGHEN Emmanuel } \\ & \end{array}$

Urologie (52.04)

Chirurgie Générale (53.02)

Chirurgie Orthopédique et Traumatologique (50.02)

Biophysique et Médecine Nucléaire (43.01)

Biologie Cellulaire (44.03)

Rhumatologie (50.01)

Biologie du Développement et de la Reproduction (54.05)

Médecine Interne (53.01)

Ophtalmologie (55.02)

Biologie Cellulaire (44.03)

Anesthésiologie et Réanimation Chirurgicale (48.01)

Chirurgie Vasculaire (51.04)

Nutrition (44.04)

Anatomie et Cytologie Pathologiques (42.03)

Dermato-Vénéréologie (50.03)

Chirurgie Plastique, Reconstructrice et Esthétique (50.04)

Anatomie et Cytologie Pathologiques (42.03)

Chirurgie Thoracique et Cardiovasculaire (51.03)

Neurochirurgie (49.02)

Psychiatrie d'Adultes (49.03)

Médecine Légale et Droit de la Santé (46.03)

Psychiatrie d'Adultes (49.03)

O.R.L. (55.01)

Cancérologie, Radiothérapie (47.02)

Biochimie et Biologie Moléculaire (44.01)

\section{PROFESSEURS PREMIERE CLASSE}

$\begin{array}{ll}\text { M. } & \text { BAQUÉ Patrick } \\ \text { M. } & \text { BATT Michel } \\ \text { M. } & \text { BÉRARD Étienne } \\ \text { M. } & \text { BERNARDIN Gilles } \\ \text { M. } & \text { BONGAIN André } \\ \text { M. } & \text { CASTILLO Laurent } \\ \text { Mme } & \text { CRENESSE Dominique }\end{array}$
Anatomie - Chirurgie Générale (42.01)

Chirurgie Vasculaire (51.04)

Pédiatrie (54.01)

Réanimation Médicale (48.02)

Gynécologie-Obstétrique (54.03)

O.R.L. (55.01)

Physiologie (44.02) 


$\begin{array}{ll}\text { M. } & \text { DE PERETTI Fernand } \\ \text { M. } & \text { DRICI Milou-Daniel } \\ \text { M. } & \text { ESNAULT Vincent } \\ \text { M. } & \text { FERRARI Émile } \\ \text { M. } & \text { GIBELIN Pierre } \\ \text { M. } & \text { GUGENHEIM Jean } \\ \text { Mme } & \text { ICHAI Carole } \\ \text { M. } & \text { LONJON Michel } \\ \text { M. } & \text { MARQUETTE Charles-Hugo } \\ \text { M. } & \text { MARTY Pierre } \\ \text { M. } & \text { MOUNIER Nicolas } \\ \text { M. } & \text { PADOVANI Bernard } \\ \text { Mme } & \text { PAQUIS Véronique } \\ \text { M. } & \text { RAUCOULES-AIMÉ Marc } \\ \text { Mme } & \text { RAYNAUD Dominique } \\ \text { M. } & \text { ROSENTHAL Éric } \\ \text { M. } & \text { SCHNEIDER Stéphane } \\ \text { M. } & \text { THOMAS Pierre } \\ \text { M. } & \text { TRAN Albert }\end{array}$

Anatomie-Chirurgie Orthopédique (42.01)

Pharmacologie Clinique (48.03)

Néphrologie (52-03)

Cardiologie (51.02)

Cardiologie (51.02)

Chirurgie Digestive (52.02)

Anesthésiologie et Réanimation Chirurgicale (48.01)

Neurochirurgie (49.02)

Pneumologie (51.01)

Parasitologie et Mycologie (45.02)

Cancérologie, Radiothérapie (47.02)

Radiologie et Imagerie Médicale (43.02)

Génétique (47.04)

Anesthésie et Réanimation Chirurgicale (48.01)

Hématologie (47.01)

Médecine Interne (53.01)

Nutrition (44.04)

Neurologie (49.01)

Hépato Gastro-entérologie (52.01)

\section{PROFESSEURS DEUXIEME CLASSE}

$\begin{array}{ll}\text { M. } & \text { ALBERTINI Marc } \\ \text { Mme } & \text { ASKENAZY-GITTARD Florence } \\ \text { M. } & \text { BAHADORAN Philippe } \\ \text { M. } & \text { BARRANGER Emmanuel } \\ \text { M. } & \text { BENIZRI Emmanuel } \\ \text { Mme } & \text { BLANC-PEDEUTOUR Florence } \\ \text { M. } & \text { BREAUD Jean } \\ \text { Mlle } & \text { BREUIL Véronique } \\ \text { M. } & \text { CANIVET Bertrand } \\ \text { M. } & \text { CARLES Michel } \\ \text { M. } & \text { CASSUTO Jill-Patrice } \\ \text { M. } & \text { CHEVALLIER Patrick } \\ \text { Mme } & \text { CHINETTI Giulia } \\ \text { M. } & \text { DUMONTIER Christian } \\ \text { M. } & \text { FERRERO Jean-Marc } \\ \text { M. } & \text { FONTAINE Denys } \\ \text { M. } & \text { FOURNIER Jean-Paul } \\ \text { M. } & \text { FREDENRICH Alexandre } \\ \text { Mlle } & \text { GIORDANENGO Valérie } \\ \text { M. } & \text { GUERIN Olivier } \\ \text { M. } & \text { HANNOUN-LEVI Jean-Michel } \\ \text { M. } & \text { IANNELLI Antonio } \\ \text { M. } & \text { JOURDAN Jacques } \\ \text { M. } & \text { LEVRAUT Jacques } \\ \text { M. } & \text { PASSERON Thierry } \\ \text { M. } & \text { PICHE Thierry } \\ \text { M. } & \text { PRADIER Christian } \\ \text { M. } & \text { ROGER Pierre-Marie } \\ \text { M. } & \text { ROHRLICH Pierre } \\ \text { M. } & \text { RUIMY Raymond } \\ \text { Mme } & \text { SACCONI Sabrina } \\ \text { M. } & \text { SADOUL Jean-Louis } \\ & \end{array}$

Pédiatrie (54.01)

Pédopsychiatrie (49.04)

Cytologie et Histologie (42.02)

Gynécologie Obstétrique (54.03)

Chirurgie Générale (53.02)

Cancérologie - Génétique (47.02)

Chirurgie Infantile (54-02)

Rhumatologie (50.01)

Médecine Interne (53.01)

Anesthésiologie Réanimation (48.01)

Hématologie et Transfusion (47.01)

Radiologie et Imagerie Médicale (43.02)

Biochimie-Biologie Moléculaire (44.01)

Chirurgie plastique

Cancérologie ; Radiothérapie (47.02)

Neurochirurgie (49.02)

Thérapeutique (48-04)

Endocrinologie, Diabète et Maladies métaboliques (54.04)

Bactériologie-Virologie (45.01)

Gériatrie (48.04)

Cancérologie ; Radiothérapie (47.02)

Chirurgie Digestive (52.02)

Chirurgie Thoracique et Cardiovasculaire (51.03)

Anesthésiologie et Réanimation Chirurgicale (48.01)

Dermato-Vénéréologie (50-03)

Gastro-entérologie (52.01)

Épidémiologie, Économie de la Santé et Prévention (46.01)

Maladies Infectieuses ; Maladies Tropicales (45.03)

Pédiatrie (54.01)

Bactériologie-virologie (45.01)

Neurologie (49.01)

Endocrinologie, Diabète et Maladies Métaboliques (54.04) 
M. STACCINI Pascal

M. TROJANI Christophe

M. VENISSAC Nicolas
Biostatistiques et Informatique Médicale (46.04)

Chirurgie Orthopédique et Traumatologique (50.02)

Chirurgie Thoracique et Cardiovasculaire (51.03)

\section{PROFESSEUR DES UNIVERSITÉS}

M. SAUTRON Jean-Baptiste Médecine Générale

\section{MAITRES DE CONFÉRENCES DES UNIVERSITÉS - PRATICIENS HOSPITALIERS}

$\begin{array}{ll}\text { Mme } & \text { ALUNNI Véronique } \\ \text { M. } & \text { AMBROSETTI Damien } \\ \text { Mme } & \text { BANNWARTH Sylvie } \\ \text { M. } & \text { BENOLIEL José } \\ \text { Mme } & \text { BERNARD-POMIER Ghislaine } \\ \text { Mme } & \text { BUREL-VANDENBOS Fanny } \\ \text { M. } & \text { DELOTTE Jérôme } \\ \text { M. } & \text { DOGLIO Alain } \\ \text { M. } & \text { FOSSE Thierry } \\ \text { M. } & \text { GARRAFFO Rodolphe } \\ \text { Mme } & \text { GIOVANNINI-CHAMI Lisa } \\ \text { Mme } & \text { HINAULT Charlotte } \\ \text { Mlle } & \text { LANDRAUD Luce } \\ \text { Mme } & \text { LEGROS Laurence } \\ \text { Mme } & \text { MAGNIÉ Marie-Noëlle } \\ \text { Mme } & \text { MOCERI Pamela } \\ \text { Mme } & \text { MUSSO-LASSALLE Sandra } \\ \text { M. } & \text { NAÏMI Mourad } \\ \text { M. } & \text { PHILIP Patrick } \\ \text { Mme } & \text { POMARES Christelle } \\ \text { M. } & \text { ROUX Christian } \\ \text { M. } & \text { TESTA Jean } \\ \text { M. } & \text { TOULON Pierre }\end{array}$

Mme ALUNNI Véronique

M. AMBROSETTH Damien

M. BENOLIEL José

Mme BERNARD-POMIER Ghislaine

Mme

$\mathrm{M}$.

M.

M.

$\mathrm{Mme}$

Mme

$\mathrm{Mm}$

Mme

Mme

M.

M.

Mme

M. TOULON Pierre
Médecine Légale et Droit de la Santé (46.03)

Cytologie et Histologie (42.02)

Génétique (47.04)

Biophysique et Médecine Nucléaire (43.01)

Immunologie (47.03)

Anatomie et Cytologie pathologiques (42.03)

Gynécologie-Obstétrique (54.03)

Bactériologie-Virologie (45.01)

Bactériologie-Virologie-Hygiène (45.01)

Pharmacologie Fondamentale (48.03)

Pédiatrie (54.01)

Biochimie et biologie moléculaire (44.01)

Bactériologie-Virologie (45.01)

Hématologie et Transfusion (47.01)

Physiologie (44.02)

Cardiologie (51.02)

Anatomie et Cytologie pathologiques (42.03)

Biochimie et Biologie moléculaire (44.01)

Cytologie et Histologie (42.02)

Parasitologie et mycologie (45.02)

Rhumatologie (50.01)

Épidémiologie Économie de la Santé et Prévention (46.01)

Hématologie et Transfusion (47.01)

\section{PROFESSEURS ASSOCIÉS}

M. HOFLIGER Philippe

Mme POURRAT Isabelle

M. PRENTKI Marc
Médecine Générale

Médecine Générale

Biochimie et Biologie moléculaire

\section{MAITRES DE CONFÉRENCES ASSOCIÉS}

$\begin{array}{ll}\text { Mme } & \text { CHATTI Kaouthar } \\ \text { M. } & \text { DARMON David } \\ \text { MI. } & \text { GARDON Gilles } \\ \text { Mme } & \text { MONNIER Brigitte } \\ \text { M. } & \text { PAPA Michel }\end{array}$

Mme CHATTI Kaouthar

M. DARMON David

Mme MONNIER Brigitte

M. PAPA Michel
Biophysique et Médecine Nucléaire

Médecine Générale

Médecine Générale

Médecine Générale

Médecine Générale 


\section{PROFESSEURS CONVENTIONNÉS DE L'UNIVERSITÉ}

M. BERTRAND François

M. BROCKER Patrice

M. CHEVALLIER Daniel

Mme FOURNIER-MEHOUAS

M. QUARANTA Jean-François
Médecine Interne

Médecine Interne Option Gériatrie

Urologie

Médecine Physique et Réadaptation

Santé Publique 


\section{Aux membres du jury,}

A Monsieur le Président du jury, le Professeur Bernard Padovani,

Vous me faites l'honneur de présider ce jury de thèse.

Je vous remercie pour votre accueil lors de mes passages dans le service, votre disponibilité et votre enseignement en imagerie thoracique.

Veuillez trouver ici le témoignage de mon profond respect.

A notre Maître et Jury de thèse, le Professeur Patrick Chevallier,

Vous me faites l'honneur d'avoir accepté de siéger à ce jury de thèse.

Je vous remercie de m'avoir accueillie dans votre service depuis mes débuts en radiologie.

Votre bienveillance et vos qualités pédagogiques en imagerie digestive diagnostique et interventionnelle m'ont aidée à progresser tout au long de mon cursus.

Soyez assuré de ma profonde reconnaissance.

A notre Maître et Jury de thèse, le Professeur Jacques Darcourt,

Vous me faites l'honneur d'avoir accepté de juger ce travail.

Je vous remercie pour vos précieux conseils et la disponibilité permanente dont vous avez fait preuve à l'occasion de mon travail de thèse.

Soyez assuré de ma profonde gratitude.

A notre Maître et Jury de thèse, le Professeur Denys Fontaine,

Vous me faites l'honneur d'être membre de mon jury de thèse.

Vous êtes unanimement reconnu pour vos compétences en neurochirurgie.

Que ce travail soit l'occasion de vous témoigner mon respect sincère. 
A notre Maître et Jury de thèse, le Docteur Fanny Vandenbos,

Je te remercie d'avoir accepté de juger ce travail.

Tes qualités pédagogiques, ton expertise en neuropathologie et ta gentillesse forcent le respect.

J’ai beaucoup apprécié travailler avec toi lors de mon passage dans le service.

\section{A ma Directrice de thèse, le Docteur Lydiane Mondot,}

Tu me fais I'honneur de diriger cette thèse.

Ce fut un réel plaisir de travailler sous ta tutelle. Je te remercie pour tes précieux conseils, tes encouragements permanents, ta disponibilité et ta gentillesse.

Trouve ici l'expression de ma sympathie et de mon estime. 


\section{A ma famille et mes amis,}

A mes parents,

Papa, merci d'avoir fait de moi ce que je suis aujourd'hui.

Cette thèse est l'aboutissement de longues années au cours desquelles ton soutien et ton amour m'ont accompagnée en permanence.

Maman, merci pour ta gentillesse, ta confiance et pour avoir partagé chaque moment important de ma vie depuis toujours.

A mon frère Jordan,

Pour ta complicité, ta tendresse et pour m'avoir supportée pendant les révisions !

A Bibi,

Merci ta présence depuis le premier jour, à l'entrée de la fac de médecine, jusqu'à aujourd'hui.

\section{A mon mari Fred,}

Tellement et si peu de choses à te dire à la fois... Merci de partager ma vie et de m'épauler à chaque instant, comme tu sais le faire.

\section{A Gérard et Sonia,}

Merci pour votre bienveillance depuis notre rencontre et pour votre soutien indéfectible.

A Margaux,

Pour tes encouragements et ta douceur.

A mes amis,

L'inco, April, Delphine, Clark, Auriane, Mathieu, Arnaud, Sarah, Seb, Myriam, Mika, Dan, et tous ceux qui se reconnaîtront.

Merci d'être toujours à mes côtés, y compris pour ce moment si attendu.

A Pierre et Valérie,

Du premier stéthoscope à maintenant, que de chemin parcouru. Merci d'être à mes côtés. 


\section{A mes maîtres d'internat,}

De mes premiers pas de radiologie à l'Archet, en passant par Pasteur, Saint-Roch, Lenval ou encore Lacassagne, merci à tous pour votre accueil, votre enseignement, votre patience pendant toutes ces années, ça a été très agréable pour moi de travailler avec chacun d'entre vous.

\section{Aux médecins nucléaires et anatomo-pathologistes,}

Pour l'accueil que j'ai reçu dans le service d'anatomo-pathologie du Pr Michiels et dans le service de médecine nucléaire de l'Archet.

\section{A mes co-internes,}

A Manue, Alix, Yann (merci pour tes workshops de vasculaire!!), Julien, Florence, Shaad, Bastien, Olivier, Elodie, Caro, Xavier, Violaine, Margaux, Alexandre Merci pour tous ces semestres passés ensemble.

\section{Aux équipes soignantes,}

Aux manipulateurs radio de jour et de nuit, du vasculaire à l'Archet, aux aides-soignants, brancardiers, secrétaires et ceux que j'oublie,

Pour votre aide et votre gentillesse tout au long de mon cursus.

\section{A ceux qui ont contribué à ce travail,}

\section{Au Professeur Staccini,}

Pour avoir réalisé mes analyses statistiques et vos qualités pédagogiques.

\section{Au Docteur Stéphane Chanalet,}

Pour votre expertise en neuroradiologie, votre bienveillance et vos conseils.

\section{A Bastien Perez,}

Pour votre aide en imagerie de perfusion et votre disponibilité. 


\section{APPORT DE L'IRM MULTIMODALE ET DE LA TEP-TDM A LA ${ }^{18}$ F-FDOPA DANS LE SUIVI DES TUMEURS CEREBRALES IRRADIEES}




\section{SOMMAIRE}

1- INTRODUCTION

page 13

2- RAPPELS

page 15

3- MATERIEL ET METHODES

page 84

4- RESULTATS

page 109

5- DISCUSSION

page 133

6- CONCLUSION

page 149

7- BIBLIOGRAPHIE

page 150

8- ANNEXE

page 170

9- TABLE DES ILLUSTRATIONS

page 171

10- LISTE DES ABREVIATIONS

page 175

11- TABLE DES MATIERES

page 178

12- SERMENT D'HIPPOCRATE

page 184 


\section{1 - INTRODUCTION}

Les tumeurs primitives d'origine gliale de haut grade et les métastases représentent plus de la moitié des tumeurs cérébrales.

Leur incidence, actuellement en augmentation, est un problème de santé publique nécessitant une expertise validée lors de Réunions de Concertation Pluridisciplinaire (RCP). Parmi les options à disposition du thérapeute, la radiothérapie est une des ressources essentielles.

Une détérioration de l'état neurologique ou encore l'apparition d'une prise de contraste avec œdème péri-lésionnel et effet de masse en imagerie peuvent compromettre l'évolution attendue après irradiation.

Se pose alors un véritable challenge diagnostique pour le radiologue, devant statuer entre des lésions radio-induites rassemblées sous le terme "radionécrose » et une progression tumorale, malgré un traitement adapté. Cette distinction est néanmoins indispensable pour une prise en charge ultérieure optimale, les mécanismes physiopathologiques impliqués et les options thérapeutiques en jeu étant radicalement différentes.

Malgré des avis non consensuels, certains paramètres morphologiques, fonctionnels et métaboliques, obtenus à partir de séquences d'Imagerie par Résonance Magnétique (IRM) cérébrale multimodale, pourraient apporter une aide précieuse pour répondre à cette problématique. La médecine nucléaire, discipline utilisant des traceurs radioactifs, occupe également une place prépondérante dans le suivi des tumeurs traitées. 
L'objectif principal de ce travail est, à partir d'une revue de la littérature, de définir quels sont les meilleurs outils en IRM multimodale pour distinguer radionécrose et récidive tumorale, dans le suivi de tumeurs cérébrales primitives et secondaires irradiées.

L'objectif secondaire est de corréler les résultats obtenus en IRM multimodale, avec ceux d'un examen de médecine nucléaire de référence, la Tomographie par émission de positons, à la ${ }^{18} \mathrm{~F}$-dihydroxyphénylalanine, couplée à la tomodensitométrie (TEP-TDM à la ${ }^{18} \mathrm{~F}$-FDOPA). 


\section{2 - RAPPELS}

\subsection{Généralités sur les tumeurs cérébrales}

2.1.1. Historique et classification histologique OMS des tumeurs du système nerveux central.

Historiquement, les prémisses de classification histologique des tumeurs cérébrales reposent sur les travaux de Cushing et Bailey en 1926 [1] et de Kernohan en 1949 [2].

L'Organisation Mondiale de la Santé (OMS) a établi, à partir de ces précédentes publications, une première classification en 1979 [3], révisée à plusieurs reprises, en 1993 [4], 2000 [5] et 2007, par l'intégration successive de données immuno-histochimiques, clinico-radiologiques et moléculaires.

La dernière version est la classification neuro-pathologique des tumeurs du système nerveux central, actuellement la plus utilisée [6]. 


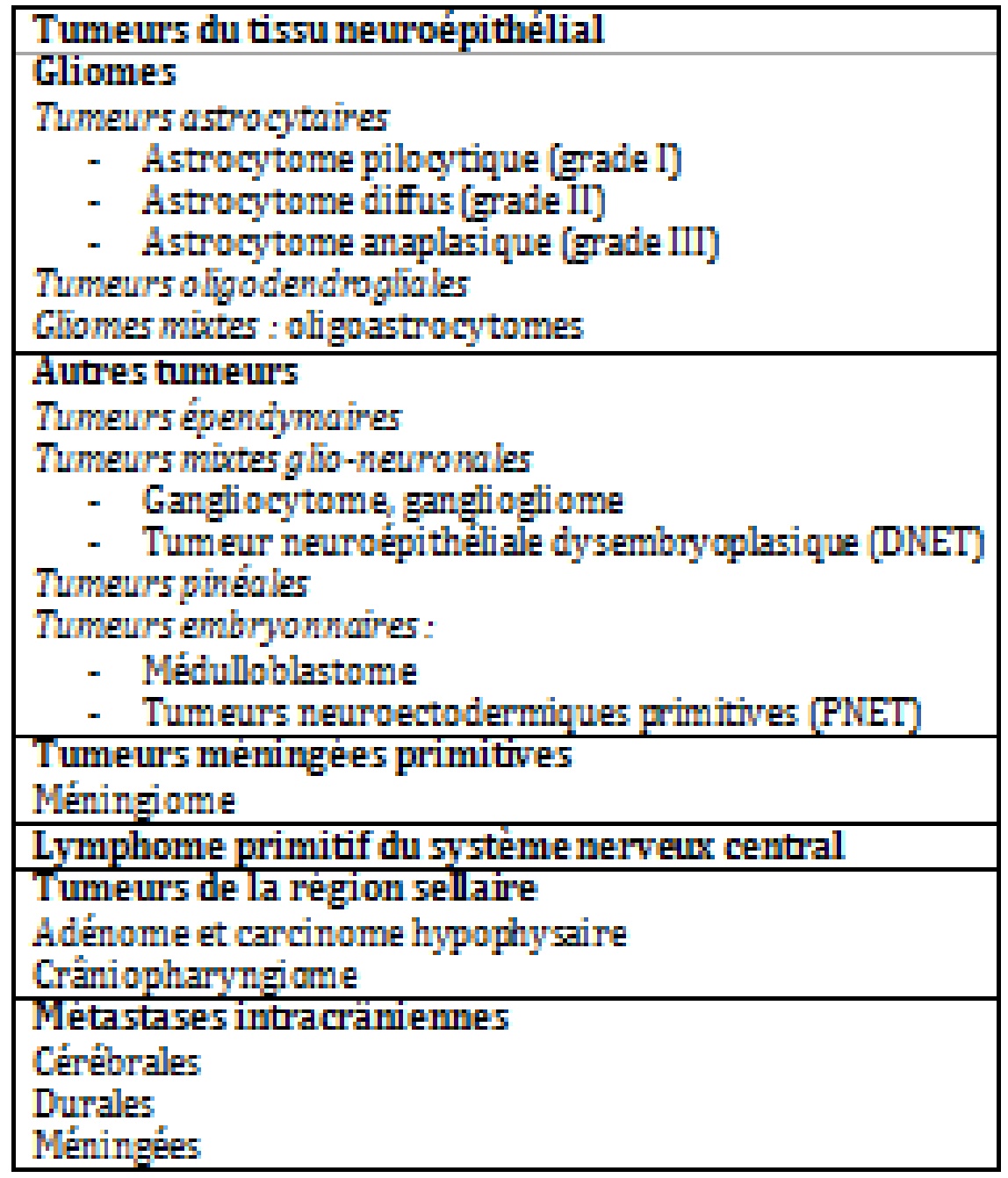

Figure 1 : Classification OMS des tumeurs du système nerveux central

Elle représente un outil commun multidisciplinaire, destiné aux pathologistes, neurochirurgiens, radiologues et thérapeutes.

Cette classification inclut à la fois les tumeurs d'origine primitive et secondaire. 
2.1.2. Les tumeurs primitives du système nerveux central (TPSNC): rappels anatomiques et histologiques. L'exemple des tumeurs gliales.

La terminologie de TPSNC est employée actuellement pour désigner tout processus tumoral primitif, siégeant dans l'encéphale, la moelle épinière, ses prolongements ou ses enveloppes [7].

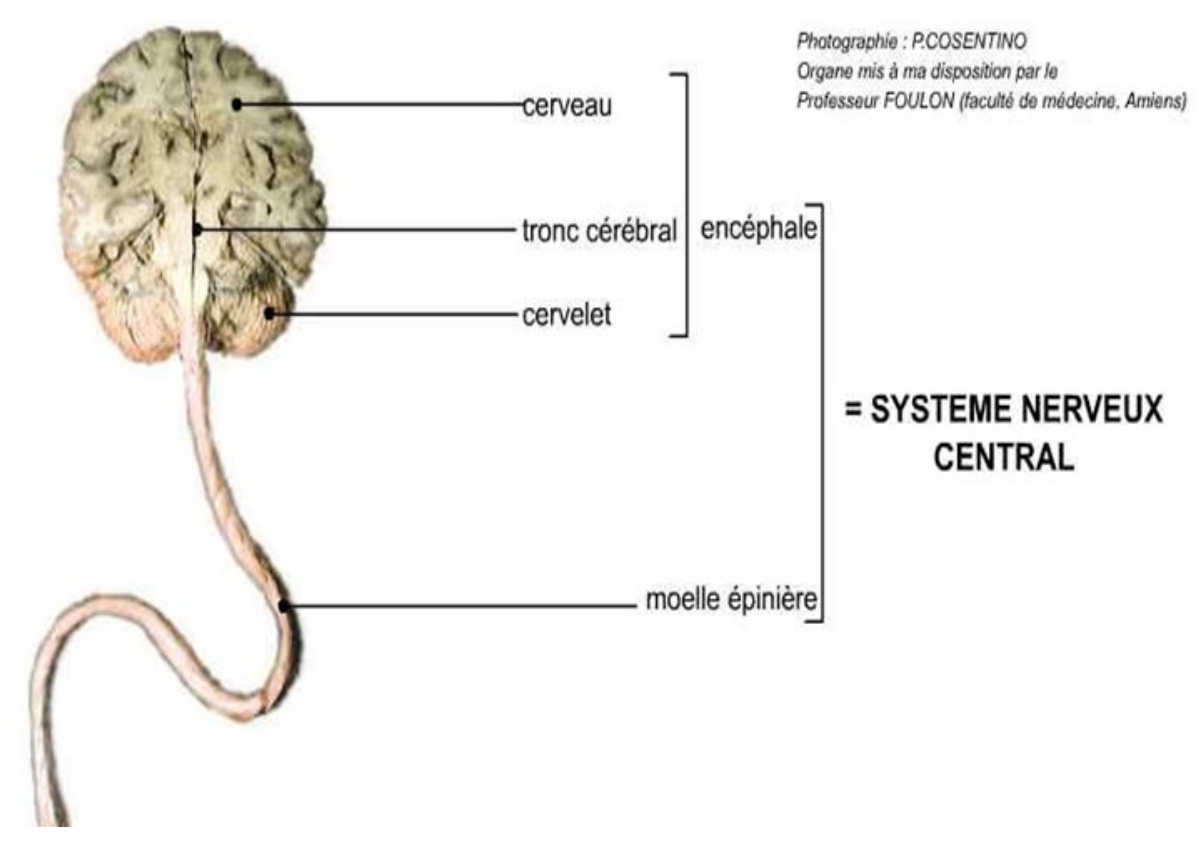

Figure 2 : Anatomie du système nerveux central

Parmi les tumeurs primitives, cette classification intègre un groupe hétérogène d'entités anatomo-pathologiques, développées à partir des différents constituants du système nerveux central. 
Elle distingue 3 principaux types de tumeurs:

- les tumeurs neuro-épithéliales qui sont développées à partir des cellules de la névroglie centrale, tissu de soutien du système nerveux, regroupant différents types cellulaires : les astrocytes, les oligodendrocytes, les cellules de la microglie et les cellules épendymaires.

- les tumeurs développées aux dépens des nerfs crâniens, représentées principalement par les neurinomes.

- les tumeurs développées à partir des méninges, enveloppes recouvrant le système nerveux, essentiellement caractérisées par les méningiomes.

Les tumeurs cérébrales primitives les plus fréquentes sont les tumeurs gliales [8], que nous allons aborder plus précisément. 


\subsubsection{Epidémiologie et facteurs de risque de tumeurs gliales}

Les tumeurs primitives d'origine gliale également appelées gliomes sont les plus fréquentes des tumeurs intracrâniennes primitives, représentant $81 \%$ des tumeurs malignes primitives de l'encéphale de l'adulte [9].

Divers facteurs de risque de survenue de gliomes ont été recensés, classés respectivement en facteurs intrinsèques et extrinsèques.

Parmi les facteurs de risque intrinsèques, les principaux connus sont le sexe masculin, l'âge, l'origine ethnique caucasienne et certains syndromes héréditaires tels que la neurofibromatose de type 1 (aussi appelée maladie de Von Recklinghausen), la sclérose tubéreuse de Bourneville ou encore le syndrome de Li-Fraumeni.

Au niveau extrinsèque, le principal facteur de risque établi concerne l'exposition aux radiations ionisantes à fortes doses.

D'autres facteurs tels que les expositions professionnelles ou l'utilisation de téléphones cellulaires ont été étudiés sans qu'aucun lien de causalité n'ait pu être démontré [10].

Par ailleurs, des facteurs protecteurs, diminuant le risque de survenue de gliomes, ont également été rapportés dans la littérature, tels que l'antécédent de manifestations allergiques ou la présence d'un terrain atopique. 


\subsubsection{Grading des tumeurs gliales \\ a- Selon la classification OMS de 2000}

L'analyse du pathologiste repose sur des critères purement histologiques, et notamment le type cellulaire prédominant, pouvant être de nature astrocytaire et/ou oligodendrogliale.

Ainsi, trois types de gliomes sont définis : les astrocytomes, les oligodendrogliomes et les gliomes mixtes appelés oligo-astrocytomes.

\begin{tabular}{|c|c|}
\hline Tumeurs astrocytaires & \\
\hline Astrocytome à cellules géantes sous-épendymaire & Grade I \\
\hline Astrocytome pilocytique & Grade I \\
\hline Astrocytome pilocytique, variant pilomyxoïde & Grade II \\
\hline Astrocytome diffus & Grade II \\
\hline Fibrillaire & \\
\hline Protoplasmique & \\
\hline Gémistocytique & \\
\hline Astrocytome anaplasique & Grade III \\
\hline Xanthoastrocytome pléiomorphe & Grade II \\
\hline Glioblastome & Grade IV \\
\hline Glioblastome à cellules géantes & Grade IV \\
\hline Gliosarcome & Grade IV \\
\hline Gliomatose & Grade III \\
\hline Tumeurs oligodendrogliales & \\
\hline Oligodendrogliome & Grade II \\
\hline Oligodendrogliome anaplasique & Grade III \\
\hline Tumeurs oligoastrocytaires & \\
\hline Oligoastrocytome & Grade II \\
\hline Oligoastrocytome anaplasique & Grade III \\
\hline
\end{tabular}

Figure 3: Principaux types de gliomes selon la classification de l'OMS 2007 (d'après D. Figarella-Branger, 2008) 
Après avoir défini le type histologique, l'étape suivante concerne l'identification du grade de malignité, à partir de cinq critères : la densité cellulaire, les atypies nucléaires, l'activité mitotique, l'existence d'une hyperplasie endothélio-capillaire et la présence de nécrose.

Ainsi quatre grades de malignité, de gravité croissante, ont été définis selon la classification OMS [5].

\begin{tabular}{|c|c|c|c|}
\hline Gliomes & Grade & Présence de & Absence de \\
\hline Astrocytome diffus & II & $\begin{array}{l}\text { Atypies nucléaires } \\
1 \text { mitose }\end{array}$ & PEC, nécrose \\
\hline Astrocytome anaplasique & III & $\geq 2$ mitoses & PEC, nécrose \\
\hline Glioblastome & IV & $\begin{array}{l}\text { Atypies nucléaires } \\
\text { Mitoses } \\
\text { PEC et/ou nécrose }\end{array}$ & - \\
\hline Oligodendrogliome & II & $\begin{array}{l}\text { Atypies nucléaires } \\
\text { Rares mitoses (nombre non précisé) }\end{array}$ & $\mathrm{PEC}$, nécrose \\
\hline Oligoendrogliome anaplasique & III & $\begin{array}{l}\text { Nombreuses mitoses } \\
\text { et/ou PEC (nécrose possible) }\end{array}$ & - \\
\hline Oligoastrocytome & II & $\begin{array}{l}\text { Atypies nucléaires } \\
\text { Rares mitoses }\end{array}$ & PEC, nécrose \\
\hline Oligoastrocytome & III & $\begin{array}{l}\text { Atypies nucléaires } \\
\text { Mitoses nombreuses et/ou PEC }\end{array}$ & Nécrose \\
\hline Oligoastrocytome & IV & $\begin{array}{l}\text { Atypies nucléaires } \\
\text { Mitoses, PEC, nécrose }\end{array}$ & - \\
\hline
\end{tabular}

Figure 4 : Grading de la classification OMS des gliomes (d'après D. Figarella-Branger, 2012) 
Le grade I est représenté principalement par l'astrocytome pilocytique, tumeur bénigne d'évolution lente. Il atteint l'enfant, l'adolescent ou l'adulte jeune [11].

Le grade II correspond aux astrocytomes diffus, tumeurs infiltrantes, dont les limites sont plus imprécises, avec in fine un risque de transformation maligne.

Le grade III définissant les tumeurs anaplasiques peut survenir de novo ou secondairement à une tumeur de grade II. Ce sont des tumeurs agressives ne présentant pas de territoire de nécrose.

Enfin, le grade $I V$, forme la plus fréquente, représentant environ $45 \%$ des gliomes, concerne les tumeurs malignes infiltrantes appelées glioblastomes [12].

De façon similaire, elles peuvent survenir de novo (glioblastome primaire) ou être secondaires à l'évolution péjorative d'une tumeur de grade inférieur (glioblastome secondaire).

Elles sont histologiquement caractérisées par la présence de nécrose et/ou d’hyperplasie endothélio-capillaire.

Leur pronostic est sombre, avec une survie moyenne de douze mois environ [13]. 


\section{b- Selon la classification de l'Hôpital Saint-Anne}

Une autre classification, celle de l'Hôpital Saint-Anne a été élaborée en 1997 [14] afin de pallier aux faiblesses rapportées de la classification OMS, notamment concernant son manque de reproductibilité inter-observateur.

Ainsi, la classification de l'Hôpital Saint-Anne s'est distinguée par la prise en compte de données d'imagerie, permettant de s'affranchir des écueils de représentativité des prélèvements en cas de tumeur hétérogène.

Elle distingue deux grades $A$ et $B$ selon l'absence ou non d'hyperplasie endothéliale et/ou la présence d'une prise de contraste en IRM.

\begin{tabular}{ll}
\hline Critère histologique et/ou d'imagerie \\
\hline Grade A & $\begin{array}{l}\text { Absence d'hyperplasie endothéliale et de prise de } \\
\text { contraste (CT ou IRM) }\end{array}$ \\
Grade B & $\begin{array}{l}\text { Présence d'une hyperplasie endothéliale et/ou d'une prise } \\
\text { de contraste (CT ou IRM) }\end{array}$ \\
\hline
\end{tabular}

CT : computerized tomography ; IRM : imagerie par résonance magnétique.

Figure 5 : Grading des oligodendrogliomes de l’Hôpital Saint-Anne 


\subsubsection{Biologie moléculaire}

D'après les neuro-pathologistes, les critères morphologiques des classifications précédemment décrites s'avèreraient insuffisants pour prédire l'évolution clinique des tumeurs ainsi que leur réponse aux traitements.

En effet, des tumeurs gliales présentant le même aspect histologique sous le microscope peuvent néanmoins évoluer de manière radicalement différente.

Ainsi, des travaux récents ont établi, à partir de biomarqueurs moléculaires, une cartographie des altérations cytogénétiques et moléculaires des gliomes, statuant d'un pronostic et d'une prise en charge thérapeutique variable.

Parmi eux, le premier marqueur épigénétique recensé concerne la présence ou non d’une méthylation du promoteur du gène 0-6-méthylguanine-DNA-méthyltransférase (MGMT), localisé sur le chromosome 10, codant pour une enzyme du même nom, impliquée dans la réparation des lésions induites à l'ADN.

La méthylation du promoteur du gène MGMT serait associée à un meilleur pronostic, compte-tenu d'une meilleure chimiosensibilité.

D'autres altérations chromosomiques et génétiques [15] impliquées dans le processus de gliomagenèse ont également été identifiées. 
Parmi elles, la délétion combinée des bras chromosomiques $1 p$ et $19 q$, reflet d'une translocation déséquilibrée $t(1 ; 19)$ ( $q 10 ; p 10)$ serait associée au phénotype oligodendroglial. Elle est observée essentiellement en cas d'oligodendrogliomes anaplasiques et de gliomes mixtes anaplasiques.

Elle serait associée à un meilleur pronostic, avec une médiane de survie rapportée qui serait 3 à 5 fois plus longue, lorsqu'elle est présente.

Une évolution spontanée plus lente de la tumeur ainsi qu'une meilleure réponse au traitement ont aussi été décrites.

Une seconde altération moléculaire est recherchée en cas de gliomes anaplasiques.

Il s'agit de la mutation IDH (Isocitrate Deshydrogenase). Elle est quasiment toujours associée à la co-délétion précédemment décrite, mais reste toutefois inconstante chez les patients non co-délétés. Elle serait corrélée à un groupe au pronostic intermédiaire.

Inversement, l'amplification du gène du récepteur du facteur de croissance épithélial EGFR (Epidermal Growth Factor Receptor), exclusive de la translocation précédemment décrite, serait quant à elle associée au phénotype astrocytaire. Sa présence serait corrélée à un pronostic plus sombre.

Enfin, en cas de glioblastome multiforme, un autre marqueur appelé $\underline{\mathrm{C}-\mathrm{Met}}$, récepteur tyrosine kinase serait impliqué dans la croissance et la progression tumorale. Il a été montré que l'activation de la voie C-Met augmenterait la résistance aux dégâts de l'ADN dans les lignées cellulaires de gliomes.

De nombreux autres biomarqueurs témoignant de l'implication de plusieurs voies de signalisation intracellulaires participent à la tumorogenèse. 


\subsubsection{Les tumeurs secondaires du système nerveux central}

L'incidence globale des métastases cérébrales est difficilement évaluable, probablement sous-estimée, avec des chiffres variables selon les études cliniques, représentant d'après les séries entre $20 \%$ et $30 \%$ de l'ensemble des tumeurs cérébrales.

Elle serait actuellement en augmentation. Celle-ci s'explique par l'évolution des thérapeutiques en oncologie et le vieillissement de la population, faisant des métastases cérébrales un véritable problème de santé publique.

Tout type de cancer peut générer le développement de lésions secondaires cérébrales [16]. Néanmoins, trois cancers primitifs sont principalement responsables de métastases cérébrales : le cancer broncho-pulmonaire responsable de 30 à 35\% de celles-ci, le cancer du sein et le mélanome, respectivement impliqués dans 10 à $20 \%$ des lésions [17].

D'autres primitifs moins fréquents sont également à garder à l'esprit : les cancers digestifs, les cancers du rein ou encore les tumeurs gynécologiques.

Dans $10 \%$ des cas, aucun primitif n'est retrouvé.

Du point de vue anatomique, l'atteinte des hémisphères cérébraux est la plus fréquemment constatée (80\%), avec préférentiellement développement des lésions à la jonction substance blanche-substance grise ; viennent ensuite l'atteinte cérébelleuse $(15 \%)$ et celle du tronc cérébral (3\%) [18]. 


\section{Points essentiels :}

La classification OMS des tumeurs du système nerveux central est la plus utilisée actuellement, intégrant à la fois des tumeurs primitives et secondaires .

Les tumeurs gliales malignes et les métastases cérébrales, représentant plus de la moitié des tumeurs cérébrales, sont les plus fréquentes, et représentent un problème de santé publique.

Les biomarqueurs moléculaires connus ont permis de mieux analyser les mécanismes impliqués dans le processus de tumorogenèse. 


\subsection{Prise en charge thérapeutique des tumeurs cérébrales}

Elle est discutée de manière pluridisciplinaire, en RCP de neuro-oncologie, après expertise du neurochirurgien, des oncologues radiothérapeutes et neurologues, de l'anatomopathologiste ainsi que du radiologue et du médecin nucléaire.

Elle comprend à la fois un traitement symptomatique, reposant principalement sur la corticothérapie et les antiépileptiques et un traitement spécifique selon le type de tumeur cérébrale concerné.

\subsubsection{Traitement symptomatique des tumeurs cérébrales}

Le premier volet thérapeutique concerne la prise en charge de l'hypertension intracrânienne, liée à l'effet de masse et l'œdème secondaire au processus tumoral.

L'exérèse chirurgicale permet une action immédiate par levée de l'effet de masse, avec amélioration des signes neurologiques dans deux cas sur trois [19].

Lorsque la chirurgie n'est pas réalisable, l'utilisation de traitements médicamenteux, essentiellement représentés par la corticothérapie, permet le contrôle de l'œdème péritumoral, par réduction de perméabilité des capillaires tumoraux anormaux.

D'autres agents tels que le mannitol ou les solutions hyper-osmotiques peuvent également être employés [20].

Parallèlement à l'hypertension intracrânienne, les patients souffrant de crises d'épilepsie peuvent bénéficier d'un traitement antiépileptique. 


\subsubsection{Traitements spécifiques des tumeurs gliales malignes}

\subsubsection{Traitement chirurgical}

L'exérèse optimale est recommandée, c'est-à-dire la plus large possible.

Les critères d'opérabilité sont l'âge du patient, son état clinique et général ainsi que les contraintes anatomiques et fonctionnelles liées à la tumeur.

La résection chirurgicale est suivie dans les 48 heures d'une IRM post-opératoire, réalisée sans et avec injection de produit de contraste, appréciant le volume du résidu tumoral éventuel présent.

En effet, sur cette exploration précoce, toute prise de contraste observée correspond à un reliquat tumoral.

La qualité de la résection chirurgicale est ainsi un facteur pronostique majeur; le volume du résidu tumoral post-opératoire influant directement sur la survie du patient [21].

Plus tardivement, la situation deviendra complexe lorsqu'il s'agira de distinguer tissu cicatriciel et tissu tumoral [22].

Il faut néanmoins garder à l'esprit que compte-tenu du caractère infiltrant des glioblastomes, leur résection est toujours incomplète à l'échelon cellulaire, puisque des cellules tumorales persistent au-delà de toute anomalie individualisée en IRM.

En cas de résection chirurgicale jugée impossible, seule une biopsie, réalisée en conditions stéréotaxiques, pour porter un diagnostic histologique sera réalisée. 
2.2.2.2. Traitement adjuvant de radio-chimiothérapie

Il doit être débuté dans un délai de 4 à 6 semaines après le traitement chirurgical.

Lorsque seule la biopsie est réalisée, il peut être instauré plus précocement.

En cas de tumeur gliale maligne, le traitement de référence de première ligne repose sur le protocole Stupp [23].

Ainsi, après résection chirurgicale, un traitement adjuvant associant radio-chimiothérapie concomitante est réalisé, comme suit.

La radiothérapie focalisée conformationnelle est délivrée en 30 fractions de 2 Gy par jour soit un total de $60 \mathrm{~Gy}$, à raison de 5 jours par semaine.

La chimiothérapie, à base de Témolozomide (Temodal ${ }^{\circledR}$ ), un agent alkylant, est administrée par voie orale, quotidiennement, une heure avant la radiothérapie, à la dose de $75 \mathrm{mg} / \mathrm{m}^{2} /$ jour pendant toute la durée de la radiothérapie, soit 42 jours.

Quatre semaines après la fin de la radio-chimiothérapie, un traitement d'entretien par chimiothérapie adjuvante, également à base de Témolozomide, est administrée à la dose de $150 \mathrm{mg} / \mathrm{m}^{2} /$ jour pendant 5 jours, lors de la première cure.

La dose est ensuite portée à $200 \mathrm{mg} / \mathrm{m}^{2} /$ jour pendant 5 jours pendant la seconde cure, si la fonction hématologique le permet.

Les cures sont ainsi répétées tous les 28 jours, pendant 6 cycles. 


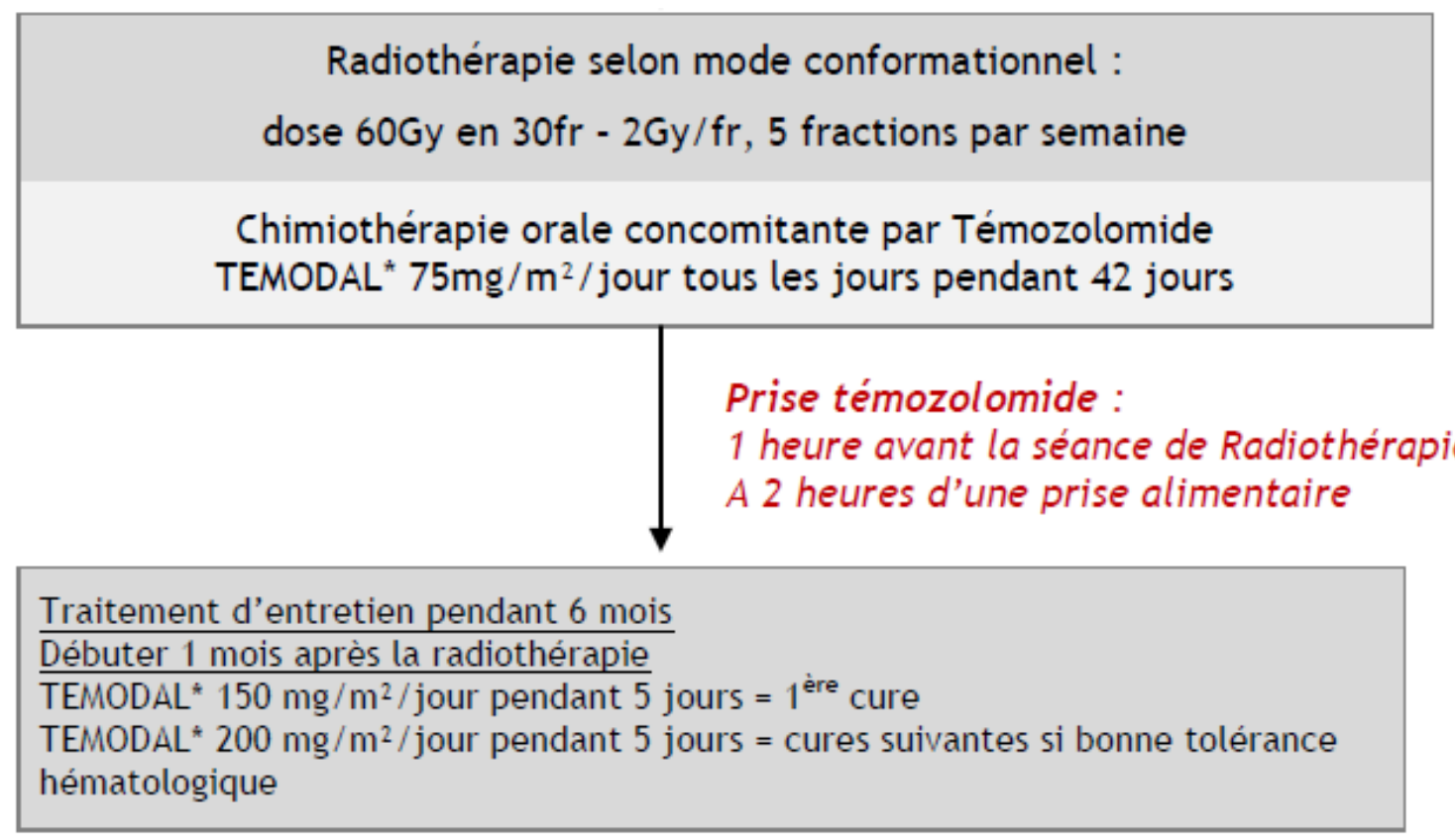

Figure 6 : Protocole Stupp (d'apèrs Anocef référentiel des tumeurs gliales)

Selon les conclusions de l'essai randomisé de Stupp, l'addition du Témolozomide à la radiothérapie a permis d'accroître la survie médiane des patients en bénéficiant, passant à 15 mois versus 12 mois, en cas de radiothérapie seule $(p<0,01)$.

Les taux de survie à 2 ans étaient respectivement de $27 \%$ en cas d'addition du Témolozomide versus $10 \%$ dans le groupe traité par radiothérapie seule.

\subsubsection{Place des implants imprégnés de Carmustine (Gliadel ${ }^{\circledR}$ )}

II s'agit de pastilles constituées de polymères biodégradables, imprégnées par un agent alkylant, la Carmustine. Ces dispositifs sont placés par le neurochirurgien au sein de la cavité d'exérèse tumorale, en cas d'exérèse complète ou quasi-complète, après obtention d'une preuve anatomo-pathologique du diagnostic de glioblastome [24]. 
La combinaison de ce dispositif au protocole de Stupp est possible, mais aucune étude randomisée n'a, à ce jour, permis d'en connaître les bénéfices [25].

\subsubsection{Traitements en cas de récidive}

La récidive (ou progression tumorale vraie) doit être impérativement distinguée d'une pseudo-progression [26].

En effet, il a été établi, dans 30\% des cas traités [27] par l'association radio-chimiothérapie concomitante, qu'une augmentation des dimensions tumorales et une modification de la prise de contraste en IRM pouvaient survenir dans les 12 semaines après le traitement.

Ce phénomène ne doit en aucun cas être considéré comme une progression tumorale réelle, mais plutôt comme un effet potentiel lié au traitement [28].

La phase d'exacerbation des lésions est transitoire et réversible, suivie par une amélioration secondaire ou une stabilisation sans traitement complémentaire [29].

Chez les patients porteurs d'une méthylation du promoteur du gène MGMT, cette réaction est plus fréquemment rapportée [30, 31].

Ainsi, en cas de récidive tumorale, après avoir écarté la possibilité d'une pseudo-progression, plusieurs options thérapeutiques peuvent être envisagées.

Une reprise chirurgicale doit être proposée lorsqu'une résection large est possible et que l'état clinique le permet. 
L'insertion d'implants de Carmustine $\left(\right.$ Gliadel $\left.^{\circledR}\right)$ a également l'AMM en seconde ligne thérapeutique.

Une autre option consistant en une ré-irradiation peut être rediscutée, en conditions stéréotaxiques, en fonction de la dose précédemment délivrée, et du délai.

Enfin, un traitement par chimiothérapie de seconde ligne peut être instauré.

L'arsenal thérapeutique est constitué de diverses molécules incluant la reprise du Témolozomide, des nitroso-urées en monothérapie (tels que la Bélustine, la Carmustine ou encore le Fotémustine) ou des schémas associant plusieurs molécules, comme par exemple le schéma PCV ou encore l'association Carboplatine-Etoposide.

En l'absence d'autre alternative thérapeutique, le Bévacizumab (Avastin ${ }^{\circledR}$ ) [32], anticorps monoclonal ciblant le Vascular Endothelial Growth Factor (VEGF) circulant [33], utilisé hors AMM, permet une survie à 6 mois sans progression de l'ordre de 40\% [34].

Il peut être administré seul ou en association avec l'irinotécan.

Cependant, un inconvénient majeur de cette molécule est lié à la survenue d'événements indésirables vasculaires hémorragiques et thrombotiques.

De plus, l'évaluation de la réponse thérapeutique au Bévacizumab en imagerie est difficile.

En effet, une diminution de rehaussement lésionnel sous traitement, par inhibition de la néo-angiogenèse peut parfois faire méconnaître une véritable progression tumorale. 


\subsubsection{Traitements spécifiques des métastases cérébrales}

La chirurgie et la radiothérapie constituent les deux principaux piliers thérapeutiques, en cas de métastases cérébrales [35].

L'objectif principal consiste en une amélioration de la qualité de vie des patients, associée dans la mesure du possible, à un allongement de leur survie.

\subsubsection{Traitement chirurgical}

II peut être envisagé à la fois en situation curative et palliative.

La chirurgie occupe un rôle majeur permettant une diminution rapide de la symptomatologie par levée de l'effet de masse, un meilleur contrôle local et une confirmation histologique du diagnostic [36].

Elle reste ainsi la modalité privilégiée lorsque la lésion est unique, d'autant plus que le patient est jeune (âge $<65$ ans), en bon état général (indice de Karnofsky $\geq 70 \%$ ) et que la tumeur primitive est bien contrôlée [37].

\subsubsection{Radiothérapie}

\subsection{Radiothérapie stéréotaxique}

II s'agit d'une technique d'irradiation de haute précision, de l'ordre du millimètre, utilisant de multiples faisceaux, permettant de délivrer des doses élevées de radiothérapie avec un fort gradient de dose. 
Elle est réalisée en conditions stéréotaxiques, pouvant être délivrée soit en une fraction unique, définissant la radiothérapie mono-fractionnée en conditions stéréotaxiques (RMCS) aussi appelée "radio-chirurgie ", soit en plusieurs fractions (jusqu'à 10) alors appelée radiothérapie hypo-fractionnée en conditions stéréotaxiques (RHCS) [38,39].

Différents systèmes existent pour réaliser cette irradiation tels que le Cyber Knife ${ }^{\circledR}$, le Gamma Knife ${ }^{\circledR}$ ou encore la radiothérapie pendulaire par mini-faisceaux de rayons $X$ de haute énergie en conditions stéréotaxiques.

Les doses employées sont très variables selon les données de la littérature, fonction à la fois du volume de la lésion cérébrale à traiter et de la proximité des organes à risque.

Elle peut être proposée [40] :

- après exérèse considérée macroscopiquement comme totale d'une métastase cérébrale symptomatique et/ou volumineuse, afin d'allonger la survie et de permettre un meilleur contrôle local tumoral.

- après découverte de 3 à 5 métastases cérébrales, de taille inférieure à $3 \mathrm{~cm}$, peu ou pas symptomatiques, d'apparition récente ou en progression malgré un traitement systémique. L'objectif étant de décaler l'irradiation pan-encéphalique et de permettre une meilleure qualité de vie, en préservant le statut neurocognitif de ces patients. 
- en combinaison avec une irradiation pan-encéphalique, afin d'optimiser le contrôle local, mais sans augmentation de la survie, avec une détérioration possible de la qualité de vie et des fonctions cognitives.

Ces considérations ont été récemment remises en cause notamment concernant le nombre de métastases cérébrales pouvant être traitées.

Ainsi, plusieurs publications ont rapporté des séries de patients présentant plus de 10 lésions secondaires traitées par cette technique [41,42].

En revanche, il reste difficile d'apprécier la place de cette option thérapeutique en cas de tumeur de petite taille, de principe opérable, et de savoir s'il faut préférer cette technique à la chirurgie [40].

\subsection{Radiothérapie conformationnelle}

L'irradiation encéphalique en totalité est la base du traitement des métastases cérébrales multiples, lorsque leur nombre est supérieur à 4 [43]. Elle est également proposée en cas d'atteinte méningée ou dans un contexte palliatif.

\subsubsection{Chimiothérapie}

Elle permet une action à la fois sur les métastases cérébrales et les autres localisations secondaires systémiques. 
Son efficacité est fonction du type histologique de la tumeur primitive.

Ainsi, en dehors des tumeurs particulièrement chimio-sensibles que sont les tumeurs germinales et le cancer broncho-pulmonaire à petites cellules, elle garde peu de place en première intention dans le traitement des métastases cérébrales, compte tenu d'une mauvaise diffusibilité à travers la barrière hémato-encéphalique.

\subsubsection{Thérapies ciblées}

Avec une meilleure connaissance des mécanismes tumoraux, de nouvelles molécules ont été intégrées à la stratégie thérapeutique en cas de métastases cérébrales.

Parmi elles, citons par exemple, les inhibiteurs de tyrosine kinase et les anti-angiogéniques. 


\section{Points essentiels :}

Discutée de manière pluridisciplinaire, en RCP, la prise en charge thérapeutique des tumeurs cérébrales inclut à la fois un volet symptomatique et spécifique selon le type tumoral.

En cas de tumeur gliale maligne, elle repose en première intention, sur une exérèse chirurgicale optimale suivie d'une radio-chimiothérapie concomitante adjuvante et d'une chimiothérapie d'entretien par Témolozomide, conformément au « protocole Stupp ».

Dans le cas de métastases cérébrales, la stratégie est non univoque, fonction du nombre de lésions.

Actuellement, la chirurgie et la radiothérapie stéréotaxique sont les modalités thérapeutiques privilégiées.

Lorsqu'il s'agit de lésions multiples, l'irradiation pan-encéphalique peut également être proposée. 


\subsection{Evolution lors du suivi post-thérapeutique}

Lors du suivi des tumeurs cérébrales primitives et secondaires, le thérapeute peut être confronté soit à une progression tumorale, soit à des phénomènes nécrotico-inflammatoires secondaires au traitement par radiothérapie mieux connus sous le terme de « radionécrose ».

\subsubsection{Progression tumorale}

Elle se définit par l'apparition d'une nouvelle lésion ou l'augmentation progressive de taille d'une lésion existante lors du suivi.

Elle s'accompagne quasi-constamment d'une détérioration de l'état clinique.

Il faut néanmoins rester prudent en cas de tumeur gliale maligne, car comme il a déjà été rappelé, une réaction peut survenir dans les 12 semaines suivant la fin du traitement par radiothérapie, appelée « pseudo-progression ».

Cette dernière doit être considérée comme un effet potentiel lié au traitement et ne doit pas amener à modifier la prise en charge du patient [44].

Par analogie, en cas de métastases cérébrales, une augmentation transitoire de volume, supérieure à $20 \%$ du volume pré-thérapeutique, rencontrée chez un tiers des patients, a été rapportée, sans impact diagnostique péjoratif.

Celle-ci peut survenir dès 6 mois après le traitement et durer jusqu'à 15 mois [67]. 


\subsubsection{Radionécrose}

Il s'agit de la principale complication redoutée, liée à la toxicité retardée de la radiothérapie, généralement irréversible et progressive [45].

Elle peut survenir de quelques mois jusqu' à plusieurs années après l'irradiation [46].

\subsubsection{Physiopathologie}

La physiopathologie des lésions radio-induites reste à ce jour controversée ; les mécanismes liés à son développement n'étant que partiellement élucidés.

Il reste néanmoins admis que la radionécrose implique l'association de plusieurs lésions anatomo-pathologiques.

Initialement survient une atteinte ischémique vasculaire touchant les artères de petit et moyen calibre, à l'origine d'une vasodilatation avec augmentation de la perméabilité capillaire, responsable d'un œdème vasogénique [47].

Suivent alors des épaississements pariétaux vasculaires par hyalinisation et une nécrose pariétale fibrinoïde avec survenue de thrombi.

L'ensemble de ces lésions conduit ainsi à une nécrose tissulaire [48] extensive, prenant un aspect serpigineux.

II s'y associe des lésions gliales et de la substance blanche [49], compte tenu de l'extrême radiosensibilité des oligodendrocytes, induisant des lésions de démyélinisation autour des foyers nécrotiques. 
Des modifications du système enzymatique fibrinolytique [50] et des mécanismes immunologiques basés sur le développement d'anticorps dirigés contre les cellules gliales lysées par l'irradiation [51] sont également impliquées. 

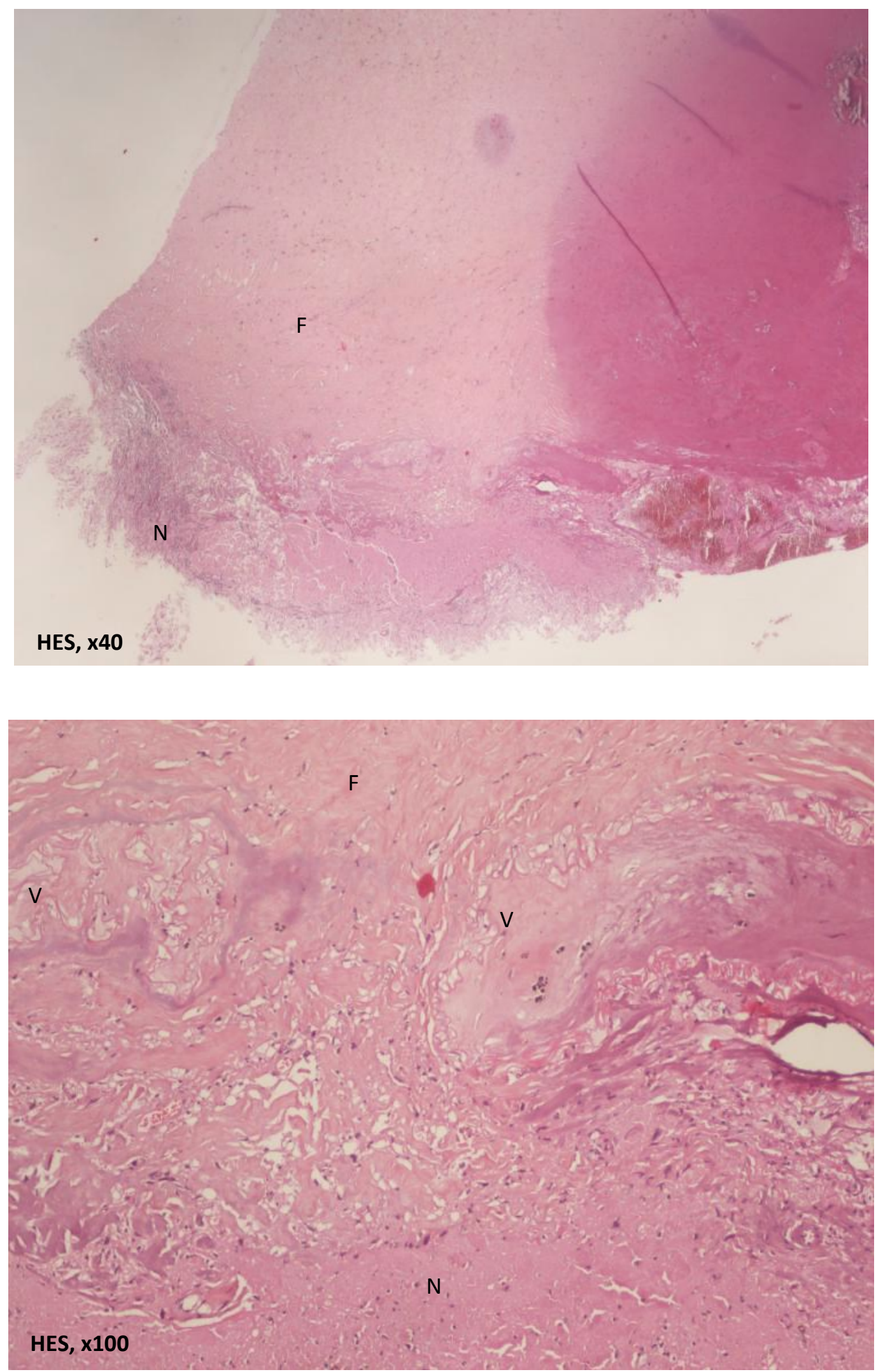

Figure 7 : Aspect anatomo-pathologique observé en cas de radiolésion

$\mathbf{N}$ : Nécrose, $\mathbf{F}$ : Fibrose et $\mathbf{V}$ : Vaisseaux pathologiques 


\subsubsection{Facteurs de risque}

Ils sont variables selon le type de radiothérapie utilisée.

En cas de radiothérapie conventionnelle, les facteurs favorisant de survenue de radionécrose recensés sont multiples. Le principal facteur retenu est la dose totale d'irradiation reçue.

D'autres facteurs à considérer sont le volume d'irradiation, le fractionnement et l'étalement du traitement [52].

D'après Ruben et al [53], la combinaison avec une chimiothérapie augmenterait également le risque de survenue de radionécrose.

En effet, certaines molécules favoriseraient le développement de lésions radio-induites telles que les sels de platine, la Doxorubicine, le Méthotrexate ou encore le Témolozomide.

Par ailleurs, en cas de radio-chirurgie, le principal facteur de risque rapporté dans l'étude de Blonigen et al [54], était le volume de tissu cérébral sain irradié à la dose de 12 Gy $\left(\mathrm{V} 12>8 \mathrm{~cm}^{3}\right)$

Par opposition, il existerait un rôle protecteur de la radiothérapie hypo-fractionnée en conditions stéréotaxiques.

Une irradiation antérieure et le sexe masculin étaient aussi des facteurs prédisposant [55] à considérer. 


\subsubsection{Modalités de diagnostic final}

L'imagerie morphologique étant souvent prise à défaut dans le diagnostic différentiel entre radionécrose et récidive tumorale, le diagnostic peut être porté soit selon l'évolution clinicoradiologique, soit en anatomo-pathologie.

Dans le premier cas, les patients qu'ils soient symptomatiques ou non, présentent en imagerie des lésions mimant une récidive tumorale, à la différence que sans traitement oncologique spécifique ajouté, il existe une stabilité voire même une régression des lésions.

Dans le second cas, le diagnostic est porté histologiquement par biopsie chirurgicale stéréotaxique [56] ou exérèse en cas de lésion symptomatique. 


\subsubsection{Modalités thérapeutiques en cas de radionécrose}

A l’heure actuelle, il n'existe pas de traitement médical spécifique.

L'utilisation de corticostéroïdes reste le standard thérapeutique, afin de diminuer l'œdème péri-lésionnel et la réaction inflammatoire associés aux lésions de radionécrose.

L'oxygénothérapie hyperbare [57] peut aussi être envisagée en cas de lésions radio-induites [58].

Des données récentes ont mis en avant le rôle essentiel du Bévacizumab dans le traitement de la radionécrose [59], compte tenu de ses propriétés anti-angiogéniques [60].

Du point de vue physiopathologique [61], VEGF serait sécrété par certains types d'astrocytes, présents dans le tissu péri-nécrotique, expliquant l'angiogenèse et l'œdème péri-lésionnel observé en cas de radionécrose.

Les patients atteints de radionécrose peuvent en outre bénéficier de nouvelles thérapies comme la thermothérapie par laser interstitiel (LITT).

Enfin, la résection chirurgicale peut parfois être nécessaire en cas de lésion symptomatique ou de doute diagnostique entre une radionécrose et une récidive tumorale, sous réserve que la lésion soit située dans une zone anatomique non fonctionnelle et accessible chirurgicalement. 


\section{Points essentiels :}

L'évolution des tumeurs cérébrales primitives et secondaires traitées par radiothérapie est variable.

Dans les 12 semaines suivant l'irradiation de tumeurs gliales, une réaction réversible appelée "pseudo-progression " mimant une progression tumorale vraie peut survenir, et ne doit pas piéger le clinicien et le radiologue.

La radiothérapie cause non seulement des effets toxiques précoces mais aussi retardés comme la radionécrose, impliquant l'intrication de mécanismes ischémiques vasculaires, de lésions gliales et de modifications enzymatiques et immunologiques.

Certains facteurs prédisposant sa survenue sont à connaître.

Son diagnostic est établi selon l'évolution clinico-radiologique ou sur données anatomopathologiques.

Les ressources thérapeutiques à disposition sont diverses.

Ainsi, le clinicien peut avoir recours aux corticoïdes, au Bévacizumab, à l'oxygénothérapie hyperbare ou encore opter pour une résection chirurgicale, si nécessaire. 


\subsection{Suivi des tumeurs cérébrales irradiées par IRM multimodale}

L'IRM multimodale [62] est un examen de référence, réalisée environ tous les trois mois après traitement de tumeurs cérébrales.

Elle repose sur la combinaison de séquences morphologiques conventionnelles, fonctionnelles telles que la diffusion et la perfusion, et métabolique représentée par la spectroscopie.

L'interprétation des diverses séquences et l'utilisation d'outils qualitatifs et quantitatifs sont une aide précieuse pour le radiologue devant statuer entre radionécrose et récidive tumorale.

\subsubsection{Séquences morphologiques}

En pratique, les séquences réalisées sont pondérées en T1 sans et avec injection de chélates de gadolinium.

II s'y associe des séquences pondérées en T2 écho de spin et en T2 FLAIR pour évaluer l'infiltration tumorale et l'œdème apparaissant tous deux en hypersignal.

Certaines équipes associent de manière systématique des séquences pondérées en T2 écho de gradient, à la recherche de remaniements hémorragiques. 


\subsubsection{Type de prise de contraste}

L'aspect classiquement rencontré lors du suivi en imagerie, à l'origine d'errances diagnostiques, est celui d’une lésion rehaussée après injection, accompagnée d'une zone centrale nécrotique.

La prise de contraste traduit alors la rupture de la barrière hémato-encéphalique [63].

Celle-ci est localisée le plus souvent, à proximité de la cavité d'exérèse, au voisinage du site tumoral et prédomine au sein de la substance blanche, en cas de radionécrose, compte-tenu de sa nature particulièrement vulnérable à l'atteinte vasculaire ischémique post-radique.

Cependant, malgré des types de rehaussements variables décrits dans la littérature [64], pouvant être nodulaire, linéaire ou curviligne, hétérogène prenant un aspect dit « en poivron coupé ", voire en "bulles de savon ", ou encore " en gruyère ", aucun n'est connu pour être discriminant et déterminer s'il s'agit de récidive ou de lésion radio-induite [45].

\subsubsection{Limites lésionnelles}

De même, certains auteurs [65] ont mis en évidence que des limites lésionnelles floues, plumetées seraient en faveur du diagnostic de radionécrose.

Par opposition, des limites nettes seraient plutôt observées lors d'une récidive tumorale. 


\subsubsection{Caractéristiques morphologiques propres aux métastases cérébrales}

Plusieurs critères morphologiques ont été abordés dans la littérature, afin de tenter de prédire l'évolution des métastases cérébrales irradiées.

Ainsi, Huang et al [66] ont observé qu'une augmentation du volume lésionnel après radiothérapie stéréotaxique supérieure à $65 \%$ (en comparaison au volume déterminé lors du premier contrôle post-thérapeutique par IRM) était suspecte de récidive ou de poursuite évolutive tumorale.

Ce constat était de bon pronostic, avec une médiane de survie supérieure (de 18,4 mois) chez les patients avec augmentation du volume lésionnel post-thérapeutique comparativement à ceux dont les lésions régressaient ou étaient stables (versus 16,4 mois).

D’autres études se sont intéressées à l'aspect des lésions en imagerie pondérée en T2 et en T1 après injection, avec des résultats contrastés selon les équipes.

Dequesada et al [68] ont ainsi comparé l'aire du nodule calculée en imagerie pondérée en T2 écho de spin (AT2) à celle visible sur la série pondérée en T1 après injection de gadolinium (AT1Gd). Lorsque le ratio AT2/AT1Gd (aussi appelé « quotient lésionnel ») était $\leq 0,3$, cela était prédictif de radionécrose tandis qu'un ratio > 0,6 était en faveur de récidive tumorale.

Néanmoins, une seconde étude plus récente n'a pas confirmé de tels résultats [69].

D'autres paramètres ont également fait l'objet de publications. 
L'existence d'un « mismatch T1/T2 », traduisant une absence de corrélation entre les limites lésionnelles observées en T2 et T1 après injection de gadolinium, était quant à lui suggestif de radionécrose dans l'étude de Kano et al [70].

La sensibilité et la spécificité de ce paramètre dans cette étude était respectivement de $83 \%$ et $91 \%$.

De plus, la présence d'un important œdème en hypersignal $T 2$, avec ratio volume hypersignal T2/volume lésionnel sur l'imagerie pondérée en T1 après injection de gadolinium élevé, supérieur à 10 , était prédictif de lésion radio-induite, avec une valeur prédictive positive de 92\% [71]. 


\section{Points essentiels :}

Le type de prise de contraste, observé lors du suivi des tumeurs cérébrales irradiées, ne serait pas, d'après les données de la littérature, un paramètre discriminant pour statuer entre radionécrose et récidive tumorale.

Des limites lésionnelles floues seraient en faveur du diagnostic de lésion radio-induite alors que des limites nettes évoqueraient une poursuite évolutive.

Ainsi, les caractéristiques morphologiques basées sur l'interprétation de séquences conventionnelles d'IRM présenteraient une faible spécificité pour distinguer les deux entités.

Dans le cas particulier des métastases cérébrales, l'augmentation du volume lésionnel après radiothérapie stéréotaxique par rapport au volume calculé lors du premier contrôle postthérapeutique supérieure à $65 \%$ serait en faveur d'une récidive tumorale.

D'autres paramètres comme la présence d'un " mismatch T1/T2 " ou encore l'extension de l'œè̀me péri-lésionnel suggèreraient la présence de radionécrose.

Ainsi, en complément des séquences morphologiques conventionnelles, le recours à d'autres séquences est une aide précieuse dans la démarche diagnostique.

\subsubsection{Séquences fonctionnelles}

Les séquences fonctionnelles de diffusion et de perfusion sont des outils complémentaires, utilisées en pratique courante, lors du protocole d'IRM multimodale. 


\subsubsection{Imagerie de perfusion}

Elle vise à étudier la microcirculation, c'est-à-dire l'écoulement du sang dans les réseaux capillaires des tissus et les échanges entre le sang et l'espace extravasculaire.

Ce phénomène assure la nutrition et le fonctionnement des tissus [78].

Tout état pathologique survenant occasionne des modifications fonctionnelles de la microcirculation, notamment en cas de néo-angiogenèse tumorale [79].

Celles-ci apparaîtraient plus précocement par rapport aux modifications morphologiques pouvant être observées en imagerie conventionnelle.

En effet, la prise de contraste lésionnelle observée en IRM avec injection de gadolinium témoigne seulement d'une rupture de la barrière hémato-encéphalique, mais en aucun cas de la présence d'une néo-angiogenèse.

\subsection{Méthodes de mesure de la microcirculation}

Plusieurs méthodes de perfusion sont décrites en IRM, pouvant être réalisées avec ou sans injection de produit de contraste.

Concernant les techniques sans injection, de façon simplifiée, il existe 2 types de séquences respectivement appelées ASL (Arterial Spin Labelling) et BOLD (Blood Oxygenation Level).

Leur usage peut être intéressant chez les patients allergiques aux produits de contraste ou en cas d'insuffisance rénale, afin de prévenir le risque de fibrose systémique néphrogénique. 
A propos des techniques avec injection de produit de contraste, elles sont basées sur l'utilisation d'agents paramagnétiques, les chélates de gadolinium.

On distingue deux méthodes non invasives distinctes de mesure de la microcirculation en IRM [80] avec injection de produit de contraste, basées sur l'utilisation d'agents paramagnétiques : I'imagerie de perfusion $\mathrm{T} 2 *$ et l'imagerie de perfusion T1.

\subsection{Imagerie de perfusion T2* (ou DSC-MRI)}

\section{Généralités}

La plus anciennement et couramment utilisée en pathologie cérébrale [81] est appelée « Dynamic Susceptibility Contrast- MRI (DSC-MRI).

Elle peut être réalisée à partir d'une séquence écho planar de type écho de gradient (pondérée en $\mathrm{T}^{*}$ ) ou écho de spin, synchronisée avec le début de l'injection intraveineuse en bolus du produit de contraste.

Le principe est tel que l'effet de susceptibilité magnétique du gadolinium entraîne une baisse du signal. Celle-ci est liée aux inhomogénéités de champ magnétique en rapport avec la variation de susceptibilité magnétique entre deux structures voisines.

Dans la majorité des cas, l'approche réalisée est dite de "premier passage », consistant à mesurer les variations de signal lors du premier passage de l'agent de contraste.

La diminution du signal observée, suite à l'arrivée très rapide du gadolinium dans les vaisseaux, est proportionnelle au nombre de vaisseaux présents par unité de volume et à leur diamètre, reflétant ainsi le volume sanguin cérébral (VSC) [82]. 
L'aspect caractéristique de la courbe de premier passage en imagerie de perfusion $\mathrm{T} 2{ }^{*}$ est représenté ci-dessous [83].

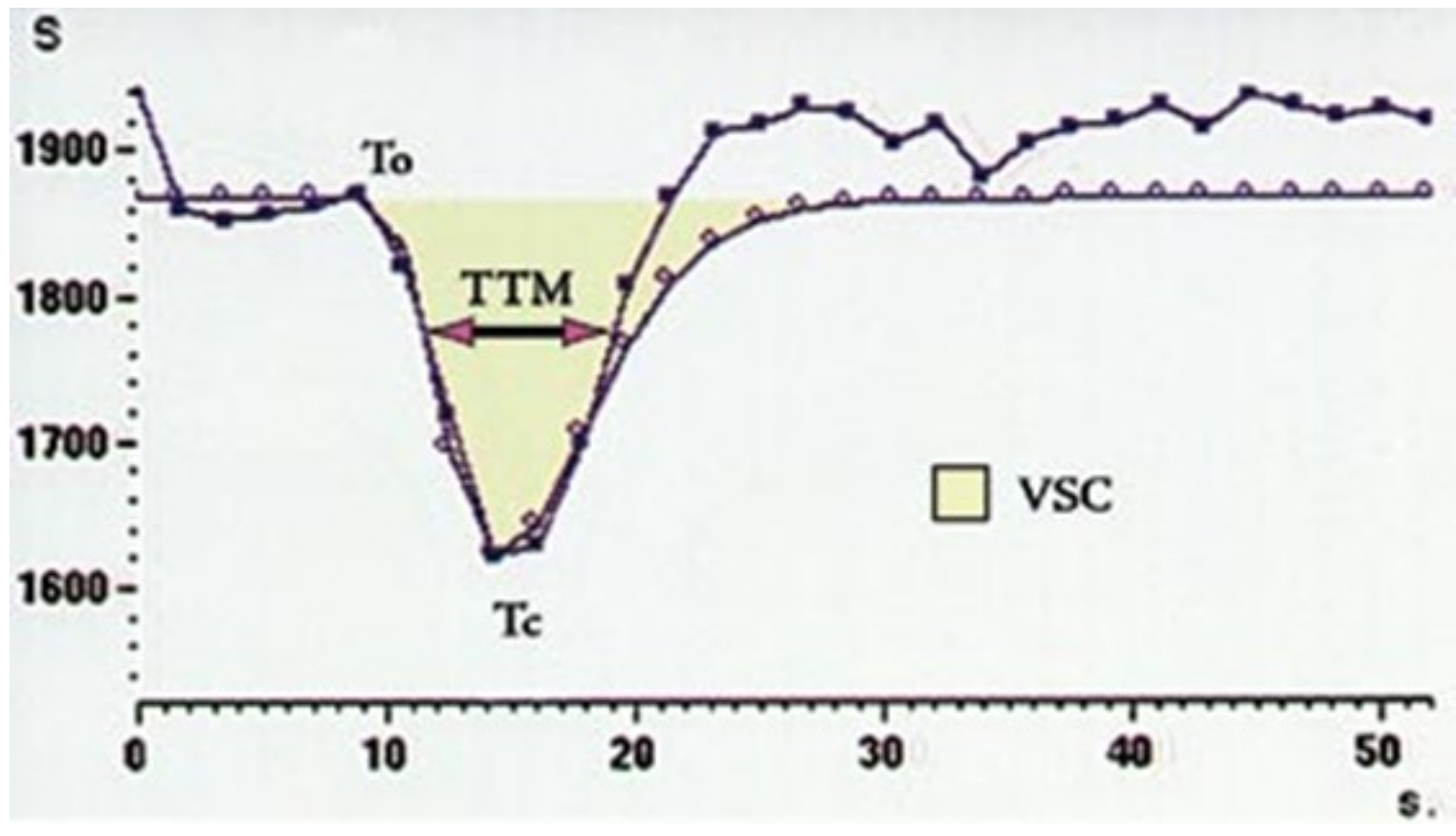

Figure 8 : Courbe de premier passage en imagerie de perfusion T2*

Initialement, on note la présence d'une ligne de base.

Après environ 10-15 secondes, temps estimé d'arrivée du bolus dans les capillaires, il existe une chute brutale de l'intensité du signal; puis, la courbe atteint un pic correspondant à la concentration maximale de l'agent de contraste dans les capillaires.

Enfin, s'effectue de façon plus ou moins rapide, un retour vers la ligne de base. 
La surface sous la courbe de premier passage est alors modélisée et peut être exprimée en termes de VSC.

Post-traitement et paramètres de suivi en imagerie de perfusion $T 2 *$ Actuellement, le recours à des logiciels de post-traitement des images permet d'obtenir des cartes paramétriques.

Les principaux paramètres mesurés en imagerie de perfusion $\mathrm{T}^{*}$ sont le volume sanguin cérébral relatif (ou $\mathbf{r}$ VSC), la hauteur du pic relative (ou $\mathbf{r}$ HP) et le pourcentage de récupération du signal (ou PSR).

\section{$\underline{\text { VSC (volume sanguin cérébral) }}$}

Il s'agit de la variable hémodynamique la plus communément utilisée en imagerie de perfusion $\mathrm{T}^{*}$.

Le résultat est présenté sous forme d'une échelle colorimétrique visuelle permettant une analyse qualitative.

Il est possible d'obtenir le calcul d'un paramètre quantitatif, le VSC, reflet de la néovascularisation tumorale.

Pour se faire, le post-traitement des images consiste à définir une région d'intérêt au sein de la zone pathologique, idéalement repérée lors de l'analyse visuelle, en zone hypervascularisée. 
La taille de cette région d'intérêt n'est pas clairement définie et varie selon les études publiées dans la littérature. Néanmoins, il convient au-delà du placement en zone d'angiogenèse maximale, d'éviter les vaisseaux corticaux et les zones de remaniements kystiques et/ou nécrotiques, pouvant fausser les valeurs obtenues [83], en s'aidant des coupes axiales acquises pondérées en T1 après injection.

Une seconde région d'intérêt est générée de façon symétrique et automatique en zone saine, au sein de la substance blanche de l'hémisphère cérébral controlatéral.

A partir de ces données, peut être calculé le ratio $r$ VSC correspondant au rapport entre le VSC maximal de la lésion et le VSC de la zone saine « en miroir ».

Pour la plupart des auteurs, un $r$ VSC compris entre 1,5 et 2 témoignerait d'une néoangiogenèse active.

Classiquement, les courbes de perfusion montrent un $r$ VSC augmenté en cas de récidive tumorale et faible lors d'une radionécrose [73], compte tenu de la présence d'une nécrose vasculaire dans ce type de lésion et non pas d'une néo-angiogenèse.

Hu et al [84] ont aussi attesté de l'efficacité de ce paramètre pour distinguer radionécrose et récidive tumorale. 
La situation n'est pas aussi simple compte-tenu de l'hétérogénéité des lésions rencontrées, de la rupture de la barrière hémato-encéphalique et de la coexistence fréquente des lésions de radionécrose et tumorales, observée dans au moins un tiers des cas [85], expliquant les zones de chevauchement dans les valeurs de perfusion.

Ce constat contribue sans doute à l'importance des variabilités inter-observateurs, pouvant aller jusqu'à 30\% [86] pour des mesures réalisées chez un même patient.

Des biais liés également aux lésions hémorragiques ou composées de mélanine peuvent être responsables d'artéfacts de susceptibilité magnétique [73].

Ainsi, Bobek et al [76] ont mis en évidence qu'en cas de récidive tumorale, le $r$ VSC moyen était de $1,46+/-0,49$ versus $0,49+/-0,38$ en cas de radionécrose $(p<0,004)$.

Pour Barajas et al [87], les valeurs rapportées de r VSC étaient significativement plus élevées dans le cas de récidives de lésions gliales de haut grade, comparativement aux radionécroses $(p<0,01)$.

Il en était de même en cas de métastase cérébrale, les valeurs de $r$ VSC étaient significativement supérieures en cas de récidive $(p<0,02)[88]$.

D’autres paramètres développés par ces auteurs ont été analysés pour différencier les lésions de radionécrose et de récidive tumorale. 


\section{$\underline{\text { r HP (hauteur du pic) }}$}

L'utilisation de ce paramètre a été suggérée initialement dans les études de Lupo et Cha [89], et plus récemment dans celles de Barajas $[87,88]$.

II représente le changement maximal de l'intensité du signal lors du passage du produit de contraste.

Ce ratio correspond ainsi au rapport entre la hauteur du pic en zone lésionnelle et celle en zone saine, selon l'équation $r \mathrm{HP}=\left(\mathrm{S}_{\mathrm{o}}-\mathrm{S}_{\min }\right)$ en zone lésionnelle $/\left(\mathrm{S}_{\mathrm{o}}-\mathrm{S}_{\min }\right)$ en zone saine Il est déterminé à partir du calcul de la différence entre l'intensité de signal au niveau de la ligne de base, avant $\mathrm{I}^{\prime}$ arrivée du contraste $\left(\mathrm{S}_{\mathrm{o}}\right)$ et l'intensité de signal minimale $\left(\mathrm{S}_{\min }\right)$ constatée lors du passage du bolus de produit de contraste, à la fois en zones lésionnelle et saine.

Les résultats rapportés, par analogie au $r$ VSC, témoignaient de valeurs moyennes plus importantes dans les récidives comparativement aux radionécroses, à la fois en cas de tumeurs gliales de haut grade $(p<0,01)$ [87] et de lésions secondaires $(p<0,02)$ [88].

II serait ainsi, d'après Cha et al [90], corrélé au r VSC et au volume capillaire tumoral. 


\section{PSR (pourcentage relatif de récupération du signal)}

Il s'agit d'un indicateur de l'intégrité de la barrière hémato-encéphalique, informant aussi des altérations de la perméabilité capillaire.

Il est défini en zone lésionnelle par l'équation $\mathbf{P S R}=\left(\mathbf{S}_{1}-\mathbf{S}_{\min }\right) /\left(\mathbf{S}_{\mathbf{0}}-\mathbf{S}_{\min }\right)$.

II correspond au rapport entre la différence d'intensité de signal maximale $\left(\mathrm{S}_{1}\right)$ et minimale $\left(\mathrm{S}_{\min }\right)$ après injection du bolus de produit de contraste et la différence d'intensité de signal au niveau de la ligne de base $\left(S_{0}\right)$, avant l'arrivée du contraste et de l'intensité de signal minimale $\left(S_{\min }\right)$ après injection.

Barajas et al [87] ont mis en évidence, chez les patients présentant une récidive tumorale, des valeurs de PSR inférieures à celles observées en cas de radionécrose, qu'il s'agisse de lésions cérébrales primitives $(p<0,01)$ ou secondaires $(p<0,05)[88]$.

II a été rapporté qu'un seuil de PSR > 87,3\% était prédictif de radionécrose, en cas d'antécédent de tumeur gliale de haut grade, avec une sensibilité de $78 \%$ et une spécificité de $76 \%$.

En cas de métastase cérébrale, la valeur seuil proposée était de 76,3\%; au-delà de cette valeur, le diagnostic était en faveur d'une radionécrose, avec une sensibilité de 95,65\% et une spécificité de $100 \%$. 


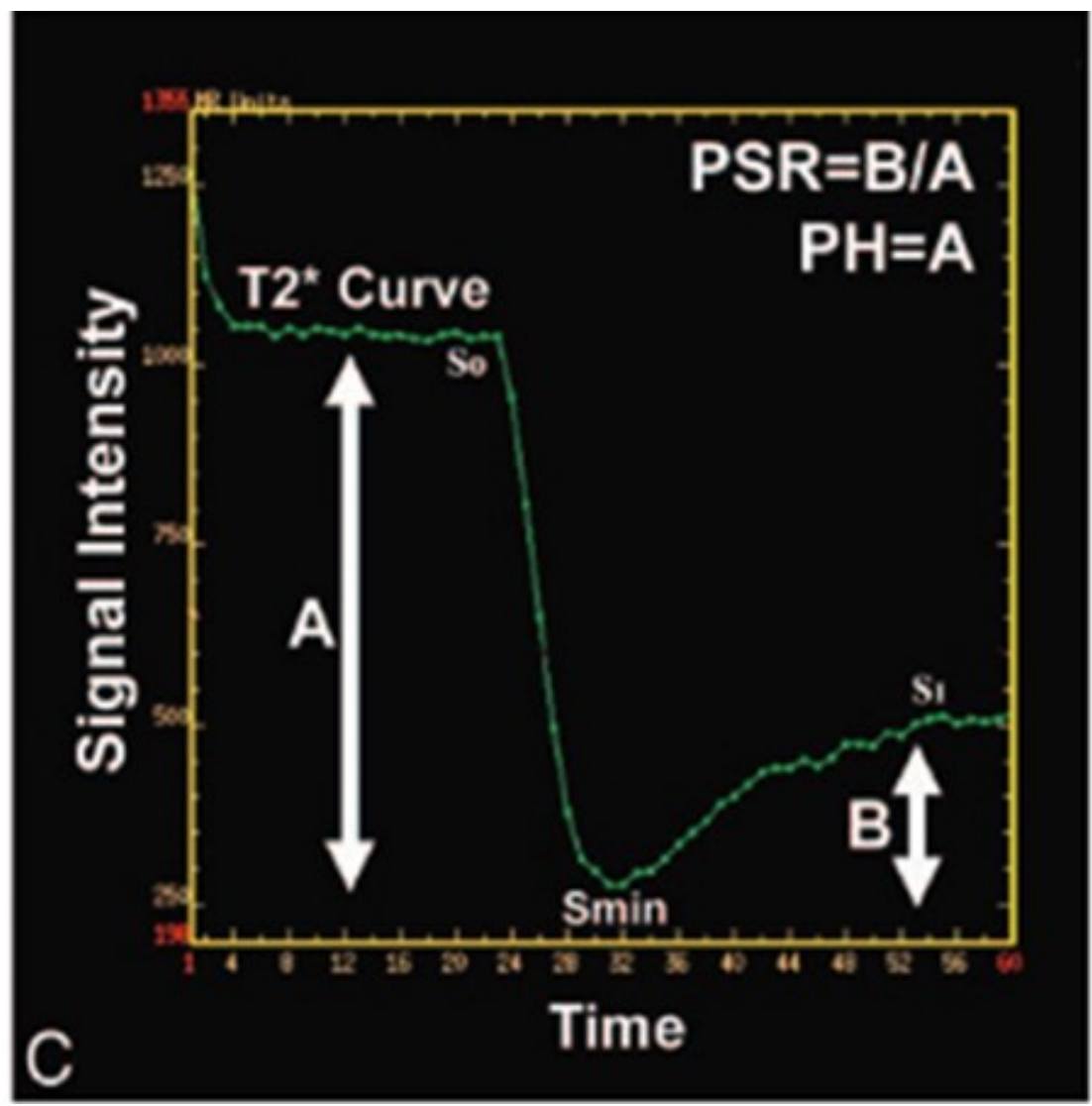

Figure 9 : Méthode de calcul des paramètres PSR et HP selon Barajas

\section{Limites}

Néanmoins, cette méthode souffre de quelques écueils secondaires à des artéfacts liés à la fois à la technique d'écho planar, source de distorsions d'images, à l'imagerie de perfusion et au traitement des images [91].

De plus, le volume sanguin cérébral peut être sous-estimé en cas de perméabilité accrue des vaisseaux tumoraux, du fait d'effets T1 associés [92, 93].

Par conséquent, ces limites ont favorisé le développement d'une autre technique, déjà beaucoup employée en dehors de la région cérébrale, appelée «Dynamic-Contrast Enhanced Imaging (DCE-Imaging), à partir de séquence pondérée en T1. 


\subsection{Imagerie de perfusion $\mathrm{T} 1$}

\section{Généralités}

Elle fait appel à l'utilisation de séquences pondérées en T1, plutôt de type écho de gradient que écho de spin.

Son principe consiste à injecter un agent de contraste pendant l'acquisition ultra-rapide (toutes les 1-2 secondes) et répétée d'images sur un ou plusieurs niveau(x) de coupe, afin de recueillir la cinétique de rehaussement du signal des tissus.

Deux phénomènes principaux se déroulent alors simultanément: la perfusion dans le réseau microcirculatoire et l'accumulation dans l'interstitium par fuite capillaire.

Ainsi, en cas de perfusion tissulaire pure, c'est-à-dire sans fuite interstitielle, on peut décomposer de manière artificielle le déroulement en trois étapes : l'entrée du produit de contraste par une artériole afférente dans le réseau capillaire, sa distribution et sa sortie par le réseau veineux.

La courbe de rehaussement cinétique tissulaire obtenue (figure 10) présentera une pente initiale, reflet du débit de perfusion tissulaire.

Celle-ci dépend du mode d'injection, du type d'agent de contraste utilisé et des caractéristiques fonctionnelles de la microcirculation locale.

Le rehaussement maximal (ou pic) renseignera sur la fraction volumique sanguine. 
Enfin, selon la vitesse de clairance rénale, l'agent de contraste va circuler à nouveau et être éliminé lentement, expliquant la pente de décroissance de la courbe.

En complément des trois étapes décrites, il faudra prendre en compte l'accumulation de produit de contraste dans l'interstitium par fuite capillaire, négligée pour raison didactique.

La cinétique de rehaussement tissulaire résulte donc d'un modèle bi-compartimental, basé sur la distribution du produit de contraste depuis l'espace micro-vasculaire jusqu' à l'espace extravasculaire.

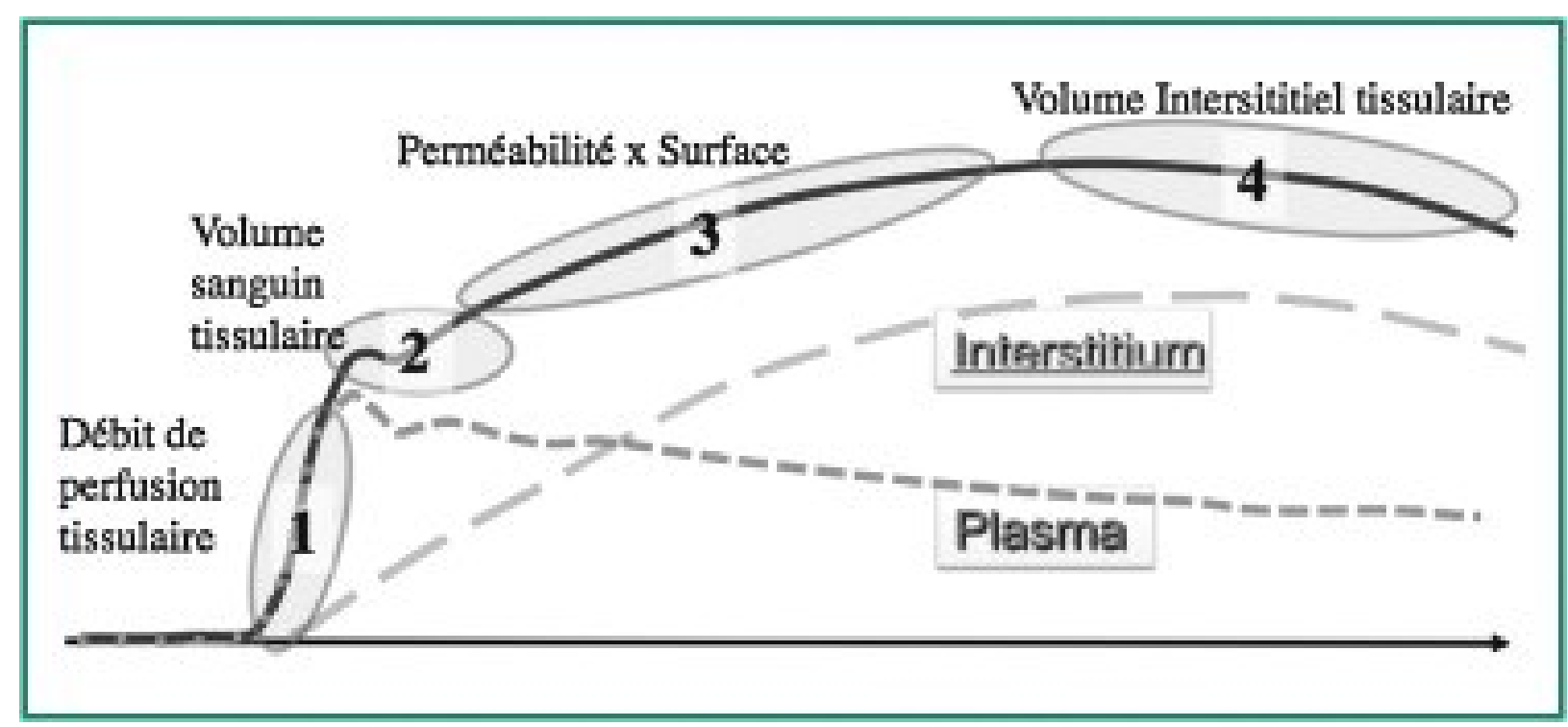

Figure 10: Signification des parties de la courbe de rehaussement tissulaire, en imagerie de perfusion T1 (d'après Cuenod, 2013)

Le rehaussement tissulaire (courbe continue) étant la sommation des composantes plasmatique et interstitielle, représentées en pointillés. 
Analyse des courbes de cinétique de rehaussement en imagerie de perfusion T1

L'analyse des courbes de rehaussement peut se faire de façon variable.

\section{Analyse qualitative}

L'analyse qualitative visuelle est rapide et simple, s'intéressant à la vitesse de la phase de rehaussement (wash-in) ou à sa cinétique (plateau, croissance lente et progressive ou encore présence d'un wash-out).

Néanmoins, compte-tenu de son manque de précision, des paramètres semi-quantitatifs et quantitatifs ont été élaborés.

\section{Analyse semi-quantitative}

Il est donc possible d'analyser la pente maximale de remplissage correspondant au rehaussement maximal (hauteur du pic).

Un autre paramètre, nécessitant l'utilisation de logiciels mathématiques, est couramment utilisé. Il s'agit de l'aire sous la courbe (aussi appelé AUC ou Area Under the Curve), correspondant à l'intégrale sous la courbe de rehaussement.

Celle-ci peut être mesurée au cours de la période précoce, appelée alors IAUGC (Initial Area Under the Gadolinium concentration Curve).

Tous ces paramètres peuvent être normalisés, par comparaison à ceux d'un tissu de référence.

Dans le cas d’une lésion cérébrale, le tissu controlatéral considéré sain servira de référence. 
Des paramètres relatifs pourront être obtenus, au moyen de ratios calculés à partir des valeurs mesurées au niveau de la zone pathologique et de la zone saine.

\section{Analyse quantitative}

Elle repose sur l'utilisation de modèles mathématiques complexes, intégrant la cinétique de rehaussement de l'artère alimentant le territoire étudié aussi appelé AIF (Arterial Input Function).

Elle permet d'étudier la perméabilité capillaire, c'est-à-dire la vitesse de déplacement d'une molécule du compartiment intravasculaire vers le compartiment extravasculaire.

Deux paramètres sont utilisés pour analyser la perméabilité capillaire.

Tout $\mathbf{d}$ 'abord, $\boldsymbol{K}_{\text {trans, }}$ constante de perméabilité, fruit du produit de la perméabilité capillaire $P$ par $S$, surface d'échanges des vaisseaux, par unité de volume $\left(K_{\text {trans }}=P x S / V\right)$, exprimé en min-1. Elle traduit la mesure du mouvement de l'agent de contraste depuis l'espace intravasculaire jusqu'à l'espace extravasculaire.

Ensuite, $\mathbf{K}_{\mathrm{ep}}$, correspondant au taux de transfert entre l'espace extravasculaire extracellulaire et le plasma. Il correspond au rapport entre $K_{\text {trans }}$ et $V_{e}, V_{e}$ étant le volume de l'espace extravasculaire extracellulaire, par unité de volume ; et s'exprime en $\min ^{-1}$. 
Paramètres de suivi en imagerie de perfusion T1

Shin et al [94] ont montré que les ratios $\mathbf{r}$ IAUGC et $\mathbf{r} \boldsymbol{K}_{\text {trans }}$ étaient concordants et significativement plus élevés en cas de récidive tumorale comparativement aux radionécroses $(p<0,05)$, montrant également, en cas de valeurs concordantes de ces paramètres, la supériorité de la technique d'imagerie de perfusion T1 par rapport à l'imagerie de perfusion T2*.

\subsubsection{Imagerie de diffusion}

Elle permet de distinguer les tissus selon de degré de mobilité des molécules d'eau le composant.

De façon simplifiée, elle repose sur l'utilisation de facteurs $b$, paramètres influant sur la pondération de l'image en IRM.

Ainsi, lorsque $b=0$, par convention, l'imagerie est dite pondérée en T2.

Plus le facteur b est élevé ( $b=1000$ ou plus), plus l'image obtenue est pondérée en diffusion, et par conséquent de façon moindre en T2.

Pour s'affranchir des effets $\mathrm{T} 2$ en imagerie de diffusion, un paramètre quantitatif appelé « coefficient apparent de diffusion » (ADC) est calculé de manière automatique par les logiciels de post-traitement d'images.

Il se traduit en chaque pixel du cerveau également de manière qualitative, par une échelle visuelle de couleur variant du bleu (en cas d'ADC bas) au rouge (en cas d'ADC élevé). 
Ce dernier permet de mesurer l'importance de la diffusion des molécules d'eau au sein des tissus.

Ainsi, lorsque celle-ci est faible, il existe une restriction de la diffusion et un coefficient ADC diminué, et réciproquement [72].

La diffusion des molécules d'eau dépend également de façon indirecte de la cellularité du tissu.

Du point de vue anatomo-pathologique, les zones de récidive tumorale se composent de noyaux pléomorphiques et d'un réseau dense de prolongements cytoplasmiques.

Par opposition, le tissu nécrosé post-radique présente une pauvreté cellulaire, avec augmentation de l'eau au sein des espaces interstitiels [73].

Ces constats sont en accord avec les résultats de plusieurs études, montrant une baisse du coefficient ADC dans les récidives tumorales par rapport aux lésions de radionécrose [74].

Hein et al [75] ont proposé un autre paramètre, le ratio d'ADC ( $\mathbf{r}$ ADC), calculé à partir de I'ADC moyen dans la zone lésionnelle, comparé à l'ADC moyen dans l'hémisphère cérébral controlatéral sain, qui serait plus discriminant que les valeurs absolues d'ADC calculées en zone lésionnelle.

Leur étude montra également que le ratio d'ADC observé chez les patients présentant des lésions de récidive était significativement plus bas ( $r A D C=1,43)$ comparativement à ceux présentant des lésions de radionécrose ( $r A D C=1,82, p<0,001)$. 
Néanmoins, les résultats proposés par d’autres études plus récentes sont variables.

Bobek et al [76] ont observé des valeurs absolues d'ADC moyen plus basses dans le groupe des récidives $\left(A D C=1,06 \pm 0,18 \times 10^{-3} \mathrm{~mm}^{2} / \mathrm{s}\right)$ comparativement au groupe des radionécroses $\left(A D C=1,13 \pm 0,13 \times 10^{-3} \mathrm{~mm}^{2} / \mathrm{s}\right)$.

Cette tendance n'était cependant pas statistiquement significative $(p=0,51)$, de même que pour les valeurs des ratios d'ADC calculés $(p=0,98)$.

Ces discordances peuvent s'expliquer d'après Chan et al [77] par le polymorphisme des lésions observées à la fois en cas de récidive tumorale et de radionécrose.

En effet, en cas de récidive, l'existence de continent nécrotique associé peut fausser les résultats.

II en est de même pour les lésions de radionécrose, dans lesquelles les phénomènes inflammatoires rencontrés influeraient sur les valeurs d'ADC.

Parfois, la présence de remaniements hémorragiques associés peut aussi mettre en péril l'imagerie de diffusion.

Bien que dans certains cas, informative, l'imagerie de diffusion semblerait donc insuffisante pour prédire l'évolution des tumeurs cérébrales après irradiation. 


\section{Points essentiels :}

L'imagerie fonctionnelle repose sur les séquences de perfusion et de diffusion.

L'imagerie de perfusion peut être réalisée sans ou avec injection de produit de contraste, dans le but d'étudier la microcirculation tissulaire.

En cas d'injection, deux techniques d'imagerie de perfusion existent : la perfusion T2* (DSCMRI) et la perfusion T1 (DCE-MRI).

La perfusion T2* prend son intérêt compte tenu de l'étude de 3 paramètres essentiels : r VSC, $r$ HP et PSR.

Les deux premiers sont augmentés en cas de récidive tumorale, contrairement au PSR diminué dans ce cas précis.

La perfusion T1 se distingue par l'étude de la perméabilité capillaire grâce à divers paramètres tels que r IAUGC ainsi que les coefficients $k_{\text {trans }}$ et $k_{e p}$.

L'imagerie de diffusion est basée sur l'étude de la mobilité des molécules d'eau constituant un tissu.

En imagerie de diffusion, le suivi des tumeurs cérébrales traitées repose principalement sur un paramètre, le ratio d'ADC ( $\mathrm{A} A D C)$. Celui-ci serait significativement plus élevé en cas de radionécrose, comparativement aux récidives.

Néanmoins, les résultats sont parfois discordants et contradictoires, selon les études réalisées. 


\subsubsection{Séquence métabolique : la spectroscopie par résonance magnétique (SRM)}

\section{Généralités}

Cette technique d'imagerie récente, utilisable en pratique quotidienne, est basée sur la détection de certains métabolites cérébraux et sur la variation de leurs concentrations.

L'analyse du métabolisme cérébral repose sur les propriétés de résonance du noyau protonique et son caractère ubiquitaire dans l'organisme.

Ainsi, à la différence de l'IRM qui permet l'obtention d'une image anatomique en coupe, la séquence de spectroscopie est recueillie sous forme de différents spectres, réalisant un ensemble de pics.

La position de chaque pic obtenu est liée à la fréquence de résonance caractéristique d'un métabolite donné.

Un paramètre essentiel à considérer lors de sa réalisation est le temps d'écho.

En effet, ce dernier va déterminer le nombre de métabolites détectables.

Ainsi, il faut souvent réaliser à la fois un temps d'écho (TE) court (30 ms) et intermédiaire (135 ms).

De même, deux types d'acquisition peuvent être réalisées : la spectroscopie mono-voxel et multi-voxel, distinctes par leur durée et le type de lésion analysée.

La spectroscopie mono-voxel permet l'étude d'un seul voxel, représentatif de la lésion, donnant ainsi une information très ponctuelle et localisée.

Son acquisition a toutefois l'avantage d'être réalisée rapidement, en 2 à 3 minutes. 
L'imagerie spectroscopique multi-voxel repose sur l'étude d'un ensemble de voxels définis sur une région d'intérêt permettant la reconstruction de cartographies des différents métabolites.

Elle donne accès à la totalité de la lésion, y compris aux zones péri-tumorales et au tissu sain adjacent [95].

En pratique, la spectroscopie est réalisée conjointement à l'IRM conventionnelle, après injection de produit de contraste afin de mieux visualiser la lésion, par acquisition monovoxel.

Le « voxel » est positionné sur la région avec prise de contraste ; lorsque celle-ci est absente, la portion la plus hétérogène est choisie.

Les rôles de la spectroscopie sont multiples, allant du diagnostic de la nature tumorale d'une lésion, à la caractérisation de tumeurs notamment gliales, à leur grading, et à l'évaluation post-thérapeutique lors du suivi.

Au-delà d'une interprétation qualitative du spectre, une analyse quantitative peut être réalisée. En effet, l'amplitude du signal est théoriquement représentative de la concentration d'un métabolite dans le volume étudié. 
D'après certains auteurs, une quantification relative, en utilisant les rapports de pics contenus dans les spectres en zone pathologique, serait mieux corrélée au diagnostic qu'en se référant à des spectres en zone saine de l’hémisphère controlatéral [96].

\section{Principaux métabolites}

A TE intermédiaire, trois principaux métabolites sont détectés en zone saine, auxquels s'ajoutent trois autres métabolites en cas de zone pathologique.

Les trois principaux métabolites concernés sont le $\mathrm{N}$-acétyl-aspartate (NAA), les composés à créatine et à choline.

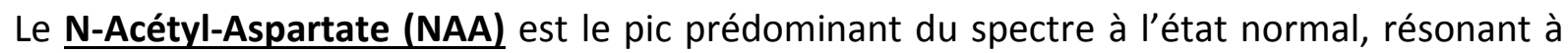
2,02 parties par million (ppm). Il s'agit d'un marqueur du fonctionnement neuronal.

La créatine/phospho-créatine $(\mathrm{Cr})$ et ses composés résonnent à 3,02 ppm.

Ce sont des témoins du métabolisme énergétique cérébral. Ce pic est utilisé comme référence compte tenu de sa stabilité théorique à l'état normal et lors de processus pathologiques.

La choline (Cho) et ses composés, résonant à 3,22 ppm, sont des précurseurs et des produits de dégradation des phospholipides membranaires.

Ils sont le reflet du métabolisme membranaire. Leur élévation traduit une prolifération cellulaire. 
Les autres métabolites rencontrés en cas de processus pathologique sont les lipides, les lactates et les acides aminés.

Les lipides, résonant à 0,9 et 1,3 ppm, visibles sous forme de pics positifs quel que soit le temps d'écho, traduisent des phénomènes de nécrose cellulaire.

Les lactates, résonant à 1,3 ppm, présentant un aspect caractéristique de doublet inversé à TE intermédiaire, sont présents en cas de métabolisme anaérobie et traduisent des phénomènes ischémiques et d'invasion macrophagique.

Les acides aminés, résonant à $0,99 \mathrm{ppm}$, sont produits lors de la dégradation de polynucléaires neutrophiles. Ils sont représentés sous forme d'un multiplet négatif à TE intermédiaire.

A TE court, d'autres métabolites peuvent être observés tels que le myo-inositol ou le complexe glutamine/glutamate.

Spectre en cas de tumeurs primitives gliales et de métastases cérébrales irradiées

Lors d'une radionécrose, une des modifications spectrales majeure est la diminution du pic de Cho $[97,98]$.

La présence de lipides, bien que fréquemment observée, n'est pas spécifique, pouvant survenir soit en cas de radionécrose, soit lors d'une évolution tumorale nécrotique [99]. 
Le phénomène de radionécrose est souvent à l'origine de débris cellulaires, contenant des acides gras, du lactate et des acides aminés.

Ainsi, certaines équipes ont identifié des pics détectés entre 2,37 et 2,4 ppm en rapport avec la présence de pyruvate ou de succinate, dans les lésions post-radiques sévères [100].

Par opposition, le spectre observé en cas de récidive tumorale associe une chute non spécifique du NAA, une diminution modérée du pic de $\mathrm{Cr}$ et une augmentation du pic de Cho, qui est d'ailleurs corrélée à l'index de prolifération $\mathrm{Ki}_{67}$, traduisant une prolifération cellulaire tumorale [101].

\section{Suivi post-thérapeutique}

Les deux principaux ratios non normalisés utilisés en pratique courante sont $\mathrm{Cho} / \mathrm{Cr}$ et Cho/NAA [102].

Dans l'étude d’Elias et al [96], le ratio Cho/NAA était le paramètre le plus discriminant pour prédire une récidive tumorale, avec une sensibilité de $86 \%$, une spécificité de $90 \%$, une valeur prédictive positive de $92 \%$ et une valeur prédictive négative de $81 \%$.

Cependant, après revue de la littérature, les seuils obtenus sont variables, probablement compte-tenu de l'hétérogénéité des lésions, du type d'acquisition réalisé ou encore des faibles effectifs inclus selon les études. 
Toutefois, les techniques d'IRM multimodale avec les séquences de perfusion et la spectrométrie amélioreraient grandement la sensibilité et la spécificité de l'examen, respectivement supérieures à 70\% et 95\% pour les séquences de perfusion [103].

\section{Limites}

Malgré ces considérations, le diagnostic différentiel entre radionécrose et récidive tumorale reste difficile à poser compte-tenu de l'intrication fréquente des deux types de lésions [104].

De plus, la topographie de certaines lésions est également un facteur limitant lorsque cellesci se situent à proximité de l'os, notamment au niveau de la base du crâne ou au contact des sinus.

Certaines lésions possédant un contingent hémorragique expliquent aussi l'altération de la qualité du spectre.

\section{Points essentiels :}

La spectroscopie permet l'analyse métabolique d'une lésion cérébrale.

Elle peut être effectuée selon une acquisition mono ou multi-voxel, à TE court ou intermédiaire, influant sur la détection des métabolites présents.

Les ratios non normalisés les plus représentatifs sont les ratios Cho/NAA et Cho/Cr.

En cas de radionécrose, le spectre présente une diminution du pic de Cho, associée selon les cas à la présence de lipides, lactates et/ou d'acides aminés.

Au contraire, une récidive tumorale se caractérise par une prolifération cellulaire et par conséquent, par une élévation du pic de choline. Cependant, l'association lésionnelle entre les deux entités est fréquemment observée, rendant la situation complexe. 


\subsection{Place de la médecine nucléaire dans le suivi des tumeurs cérébrales traitées}

L'imagerie métabolique semble également être un outil prometteur en neuro-oncologie.

Elle repose sur l'utilisation de deux modalités principales: la tomographie par émission mono-photonique (TEMP) et la tomographie par émission de positions (TEP).

\subsubsection{Généralités}

\subsubsection{La tomographie par émission mono-photonique (TEMP)}

Cette modalité utilisant des traceurs de l'activité mitochondriale tels que le 99mTc-MIBI ou le 201-Thallium est une aide précieuse dans le diagnostic différentiel entre récidive tumorale et radionécrose [105].

En effet, différentes équipes se sont intéressées aux performances diagnostiques de ces traceurs.

Ainsi, en 2006, l'équipe de Le Jeune [106] a mis en évidence des valeurs de sensibilité et spécificité respectivement de $90 \%$ et $91,5 \%$, lors de la réalisation de scintigraphies au 99mTc-sestamibi, concernant la détection d’une récidive tumorale.

Plus récemment, Matsunaga et al [107] ont confirmé l'utilité de la scintigraphie au 201-Thallium dans le suivi des tumeurs cérébrales malignes irradiées.

\subsubsection{La tomographie par émission de positons (TEP)}

Bien connue en oncologie, cette technique d'imagerie isotopique repose sur l'utilisation de traceurs marqués par des radioéléments émetteurs de positons. 
Lors de son émission par le noyau, le positon qui parcourt un trajet de l'ordre du millimètre, entre en collision avec un électron du cortège. Cette dernière génère une réaction d'annihilation, occasionnant la production de deux photons de même énergie, égale à $511 \mathrm{KeV}$, émis simultanément et en direction opposée.

La caméra détecte les coïncidences qui sont alors converties en images tomographiques, par des techniques de reconstruction et de correction d'atténuation [108].

C'est la tomographie par émission de positions (TEP).

Elle est le plus souvent actuellement couplée à un scanner X (TEP-TDM) qui permet une meilleure correction d'atténuation et une localisation anatomique plus précise.

\subsubsection{Radio-pharmaceutiques}

Divers radio-pharmaceutiques peuvent être utilisés en TEP.

Il faut distinguer les radio-pharmaceutiques utilisables en routine de ceux d'utilisation récente.

\subsubsection{La TEP au 18 Fluoro-déoxyglucose $\left({ }^{18} \mathrm{~F}-\mathrm{FDG}\right)$}

Le radio-pharmaceutique le plus utilisé en oncologie est ${ }^{18}$ F-FDG [109].

Il s'agit d'un analogue du glucose marqué au fluor 18, dont la demi-vie est de 110 minutes.

Son principe repose sur la consommation accrue de glucose par les cellules des tumeurs malignes, connu depuis les travaux de Warburg [110].

Une fois capté par les transporteurs membranaires du glucose GLUT1 et GLUT3, le deoxyglucose est transporté à l'intérieur de la cellule puis phosphorylé via l'hexokinase en 
${ }^{18} \mathrm{~F}$ - fluorodéoxyglucose-6-phosphate, bloquant son métabolisme et conduisant au piégeage du déoxyglucose dans la cellule.

Son marquage à l'aide du fluor 18 permet de quantifier son accumulation, témoin de la modification métabolique cellulaire [111].

Ce radio-pharmaceutique est donc largement utilisé en oncologie.

Cependant, pour l'imagerie cérébrale, le principal écueil de ce traceur est lié à sa fixation physiologique intense par le tissu cérébral sain.

La TEP-TDM au FDG biphasé permet de renforcer le contraste de l'hyper-métabolisme tumoral sur tissu sain [112].

Certaines études ont malgré tout montré son utilité pour distinguer récidive de gliome de haut grade et radionécrose, avec une sensibilité de $80-90 \%$ et une spécificité variant entre 50 et $90 \%$ [113] avec des résultats similaires en cas de métastases cérébrales traitées par radiothérapie stéréotaxique [114].

De plus, la fusion des images avec celles de l'IRM contribue à améliorer les performances diagnostiques de la TEP-TDM au ${ }^{18}$ F-FDG, avec une augmentation de sa sensibilité passant de $65 \%$ à $86 \%$ pour différentier radionécrose et récidive tumorale [115].

Une revue de la littérature portant sur 26 publications a comparé les performances diagnostiques de la TEMP et la TEP-TDM. 
L'analyse a permis de montrer une meilleure sensibilité de la TEP-TDM au ${ }^{18}$ F-FDG pour différentier progression tumorale et effets secondaires liés aux traitements (pseudoprogression ou radionécrose).

Par opposition, la scintigraphie au Thallium 201 présenterait une spécificité plus élevée [116].

Quoiqu'il en soit, il faut garder à l'esprit la faible résolution spatiale de la TEMP, limitant son utilisation en pratique quotidienne.

\subsubsection{Nouveaux radio-pharmaceutiques utilisés en neuro-oncologie}

Pour le suivi des tumeurs cérébrales, il semble préférable de recourir à des radiopharmaceutiques, présentant une grande stabilité in vivo et ayant une fixation faible dans le tissu cérébral sain et une accumulation dans le tissu néoplasique, indépendamment du glucose.

Les acides aminés marqués, traceurs dérivés des acides aminés, répondant à ces critères, ont une place préférentielle dans le diagnostic différentiel entre radionécrose et récidive tumorale.

Leur utilisation est sous-tendue par l'augmentation de la synthèse protéique et du transport des acides aminés par la tumeur [117], en particulier le transporteur LAT 1 (Large Aminoacid Transporter), via le complexe LAT 1/CD98, qui est surexprimé en cas de tumeurs cérébrales. 
Les trois radio-pharmaceutiques les plus utilisés sont la méthionine marquée au carbone-11 $\left(\left[{ }^{11} \mathrm{C}\right]-\mathrm{MET}\right)$, le fluoro-éthyl-tyrosine (FET) marqué au fluor-18 $\left({ }^{18} \mathrm{~F}-\mathrm{FET}\right)$ et le 6-Fluoro- $\left[{ }^{18} \mathrm{~F}\right]-\mathrm{L}-$ Dopa marqué au fluor-18 ( ${ }^{18} \mathrm{~F}$-FDOPA).Tous les trois sont des acides aminés marqués ou des analogues substrats du LAT 1.

\subsection{TEP à la $\left[{ }^{11} \mathrm{C}\right]$-méthionine}

II s'agit du traceur de référence, dont la supériorité au ${ }^{18}$ F-FDG a déjà été rapportée [118].

Cependant, il ne peut être utilisé couramment dans tous les centres compte tenu de la très courte demi-vie, égale à 20 minutes, du carbone 11 [119].

Son utilisation reste limitée car réservée seulement aux centres disposant d'un cyclotron à proximité [120, 121].

\subsection{TEP à la $\left[{ }^{18} \mathrm{~F}\right] \mathrm{FET}$}

Elle repose sur l'utilisation d'un analogue fluoré de l'acide aminé tyrosine.

En 2000, il a ainsi été montré que la fixation de la FET était plus intense et rapide en cas de lésion cérébrale maligne, comparable à celle de la méthionine marquée au [11C], avec un coefficient de corrélation de 0,98 sur 16 examens [122].

Pour Popper et al [123], il existait une hyperfixation focale et intense du traceur en cas de récidive tumorale par opposition à la fixation faible et homogène de la radionécrose. 
Dans la série de Nataf et al [124], la comparaison de cette modalité à la TEP-TDM au ${ }^{18}$ F-FDG a permis de mettre en évidence la supériorité de la TEP utilisant le FET.

En effet, les performances diagnostiques de la FET étaient de $88 \%$ de sensibilité et $80 \%$ d'efficacité versus $13 \%$ et $30 \%$.

De même, plus récemment, Galldiks et al [125] ont montré la supériorité diagnostique de cette modalité par rapport à l'IRM conventionnelle pour distinguer progression tumorale et modifications liées aux traitements.

\subsection{TEP à la ${ }^{18}$ F-FDOPA}

Le 6-Fluoro- $\left[{ }^{18} \mathrm{~F}\right]-\mathrm{L}-\mathrm{DOPA}$, analogue de la di-hydro-phénylalanine, est un acide aminé aromatique précurseur de la dopamine, marqué au fluor 18.

A la différence de la dopamine et par analogie aux autres acides aminés, il est capable de franchir la barrière hémato-encéphalique. II est ensuite converti en dopamine, via la DOPA décarboxylase.

II s'est également révélé plus sensible que le ${ }^{18}$ F-FDG [126] dans la distinction entre radionécrose et récidive tumorale.

De même que le FET, il s'impose comme une alternative intéressante à la méthionine marquée au $\left[{ }^{11} \mathrm{C}\right]$, compte tenu de la demi-vie du fluor 18 permettant sa plus large utilisation [127]. 
Connu dans l'étude des syndromes parkinsoniens grâce à la quantification de l'activité dopaminergique striatale, ce traceur a également toute sa place en neuro-oncologie depuis l'obtention d'une AMM en 2010.

Par analogie aux autres traceurs, sa réalisation repose sur la tomographie par émission de positons couplée à une tomodensitométrie pour correction d'atténuation et repérage anatomique.

Son interprétation repose actuellement sur plusieurs critères.

Le principal critère rapporté concerne l'intensité de fixation de la lésion comparativement à celle des striata, qui présentent une fixation physiologique.

L'analyse qualitative de ce paramètre est un des outils les plus discriminants pour distinguer une récidive tumorale d'une lésion radio-induite [128].

Initialement à l'initiative des cliniciens, ce score peut être côté de 0 à 3.

Ainsi, une absence de fixation en dehors de la fixation physiologique des striata correspond à un score de 0 ; a contrario, une lésion dont l'intensité de fixation serait supérieure à celle des striata, disposera du score maximal attribué.

Lizarraga et al [128] ont montré qu'une échelle visuelle supérieure ou égale à 2 était en faveur du diagnostic de récidive, avec une sensibilité de $81,3 \%$ et une spécificité de $84,3 \%$. En d'autres termes, lorsque l'intensité de fixation était égale ou supérieure à celle des striata, la suspicion de récidive était élevée. 
L'utilisation de nombreux paramètres semi-quantitatifs a également été rapportée dans la littérature [129], montrant le plus souvent des performances diagnostiques comparables. Ce constat peut s'expliquer par l'association lésionnelle fréquente observée entre radionécrose et récidive tumorale, pouvant prendre à défaut les paramètres calculés.

Cependant, récemment, une étude portant sur le suivi de métastases cérébrales après traitement par radio-chirurgie, a rapporté que le paramètre présentant la meilleure performance diagnostique correspondait au ratio de l'intensité de fixation maximale de la lésion sur la valeur maximale de fixation du bruit de fond (SUV max L/ BCK max) (SUVmax= Standardised Uptake Value maximum ; BCK max= Background maximum).

Une valeur seuil de 1,59 permettait de différencier radionécrose et récidive, avec une sensibilité de 90\% et une spécificité de 92,3\% [130].

D’autres études seraient néanmoins nécessaires pour valider les résultats rapportés par cette étude.

La TEP à la ${ }^{18} \mathrm{~F}$-FDOPA a également montré des performances diagnostiques élevées avec une meilleure spécificité $(88,89 \%$ versus $44,4 \%)$ par rapport à l'IRM conventionnelle avec injection de produit de contraste, dans la détection de récidives de gliomes traités [131]. 


\section{Points essentiels :}

L'imagerie métabolique est un outil précieux pour aider au diagnostic différentiel entre radionécrose et récidive tumorale, dans le suivi de tumeurs cérébrales irradiées.

Compte tenu de la faible résolution spatiale de la TEMP, la TEP-TDM occupe une place privilégiée en neuro-oncologie.

Plusieurs radio-pharmaceutiques peuvent être utilisés.

Le plus connu, en imagerie oncologique, est le ${ }^{18} \mathrm{~F}-F D G$, dont la fixation au niveau du parenchyme cérébral sain limite son utilisation dans cette indication.

Des acides aminés marqués, dont la fixation physiologique dans le tissu cérébral sain est faible, se sont également développés.

Parmi eux, la méthionine marquée au carbone-11 ([ $\left.\left.{ }^{11} \mathrm{C}\right]-M e t\right)$, la fluoro-éthyl-tyrosine marquée au Fluor-18 $\left(\left[{ }^{18} \mathrm{~F}\right]-\mathrm{FET}\right)$ et le 6-Fluoro-[18F]-L-Dopa $\left(\left[{ }^{18} \mathrm{~F}\right]-\mathrm{FDOPA}\right)$ sont les principaux. Le premier est peu utilisé en pratique courante, compte-tenu de la très courte demi-vie du carbone-11 comparativement à celle du fluor-18.

Actuellement, depuis son AMM en 2010 dans cette indication, la TEP-TDM à la ${ }^{18}$ F-FDOPA est la plus utilisée en pratique courante. 


\section{3 - MATERIELS ET METHODES}

\subsection{Population étudiée}

Cette étude monocentrique prospective, réalisée entre janvier 2013 et mars 2015, a porté sur tous les patients aux antécédents de tumeurs cérébrales irradiées, primitives ou secondaires, posant lors de leur suivi, le problème du diagnostic différentiel entre récidive tumorale et radionécrose secondaire au traitement.

Les patients inclus ont bénéficié d'une imagerie par IRM multimodale, incluant des séquences conventionnelles, fonctionnelles et métabolique, réalisée dans le service de radiologie de l'hôpital Pasteur au CHU de Nice.

Une étude complémentaire par TEP-TDM à la ${ }^{18} \mathrm{~F}$-FDOPA a également été réalisée chez ces patients dans le service de médecine nucléaire du Centre Antoine Lacassagne.

\subsection{Protocole d'IRM multimodale}

Toutes les images IRM ont été réalisées selon un protocole standardisé d'acquisition sur un appareil IRM General Electric HealthCare SIGNA à haut champ magnétique, d' 1,5 Tesla, HDxT, à l'aide d'une antenne 8 éléments (8 HR BRAIN).

Le patient était positionné en position supine (tête première dans le tunnel), les bras le long du corps. 


\subsubsection{Types de séquences réalisées et paramètres techniques}

Le protocole d'IRM multimodale réalisé comprenait à la fois des séquences conventionnelles, fonctionnelles et métabolique, durant au total environ 40 minutes.

\section{Séquences conventionnelles :}

-Séquences axiales pondérées en T2 (spin écho, TR : 7000ms / TE : 95,648 ms, angle de bascule : $142^{\circ}$, champ de vue : $240 \times 240 \mathrm{~mm}$, épaisseur de coupe : $4 \mathrm{~mm}$ ).

-Séquences sagittales Cube T2 FLAIR (TR: 7600 ms/ TI : 2000 ms, TE maximal, facteur de train d'échos : 200, champ de vue: 256 × $256 \mathrm{~mm}$, épaisseur de coupe : 1,2 mm).

-Séquences axiales 3D pondérées en $\mathrm{T} 1$ sans et après injection de chélates de gadolinium (TR : 500 ms / TE minimal, train d'échos : 20, champ de vue : 256 x 256 mm, épaisseur de coupe : $1,2 \mathrm{~mm})$.

\section{Séquences fonctionnelles :}

-Séquences de diffusion (TR : $7151 \mathrm{~ms}$ / TE minimal, gradients $b=0$ et $b=1000$, champ de vue : $240 \times 240 \mathrm{~mm}$, épaisseur de coupe : $4 \mathrm{~mm}$, intervalle inter-coupe : 0,4 mm).

-Séquence de perfusion 3D T1 fSPGR (fast SPoiled Gradient echo, TR : 5,3 ms / TE : 1,1 ms, angle de bascule: $30^{\circ}$, champ de vue : 240 × $240 \mathrm{~mm}$, épaisseur de coupe : $5 \mathrm{~mm}$ ).

Le protocole de perfusion T1 a été réalisé conformément aux recommandations de la QIBA (Quantitative Imaging Biomarker Alliance). 
La phase initiale comportait l'acquisition de trois phases sans injection, qui serviront de baseline à l'application du modèle pharmacocinétique, dans l'étape ultérieure de posttraitement.

A partir de la quatrième acquisition, une injection de $15 \mathrm{~mL}$ d'acide gadotérique (DOTAREM ${ }^{\circledR}$ ), par voie intraveineuse, à un débit de 3 cc par seconde était réalisée de manière dynamique, selon une résolution temporelle de 6 secondes, soit 36 phases injectées pour une durée totale de 4 minutes.

Aucun filtre supplémentaire n'était ajouté aux images obtenues puisque toute modification artificielle de l'intensité du signal d'un voxel induirait des modifications des constantes calculées par le modèle pharmacocinétique.

L'imagerie de perfusion T1 était réalisée avant l'imagerie de perfusion T2* afin de réaliser une pré-imprégnation des tissus en gadolinium.

-Séquences de perfusion T2* (spin écho, TR : $1500 \mathrm{~ms} / \mathrm{TE}: 30 \mathrm{~ms}$, champ de vue : 260x 260 $\mathrm{mm}$, épaisseur de coupe : $6 \mathrm{~mm}$, intervalle inter-coupe : $1 \mathrm{~mm}$ ).

Le protocole de perfusion $\mathrm{T}^{*}$ comportait une injection de $15 \mathrm{~mL}$ d'acide gadotérique (DOTAREM ${ }^{\circledR}$ ), selon un débit de 6 cc par seconde. Au total, 50 phases durant respectivement 3 secondes étaient réalisées pour une durée totale d'1 minute et 15 secondes.

\section{Séquence métabolique :}

-Séquence de spectroscopie mono-voxel (TR : $1500 \mathrm{~ms} / \mathrm{TE}: 35 \mathrm{~ms}$, FOV : 240 x $240 \mathrm{~mm}$, épaisseur de coupe : $15 \mathrm{~mm})$. 


\subsubsection{Post-traitement}

II a porté sur les séquences de diffusion et de perfusion, via l'utilisation des logiciels dédiés, sur une console IRM constructeur (Advantage Workstation, version 4.6).

\subsubsection{Post-traitement en imagerie de perfusion T1}

Il était réalisé à l'aide du logiciel GEN IQ, dans sa version "BRAIN ", logiciel de posttraitement de dynamique T1 dédié à l'exploration encéphalique.

Il permet de corréler la variation d'intensité de signal en IRM à la concentration en agent de contraste par l'intermédiaire d'un modèle pharmacocinétique.

Il donne ainsi accès aux cartographies fonctionnelles en relation avec le flux et la fuite de l'agent de contraste depuis le milieu intravasculaire vers le milieu extravasculaire extracellulaire.

Le logiciel détectait automatiquement l'entrée vasculaire (VIF pour Vessel Input Function).

Avant tout post-traitement, il était nécessaire de s'assurer préalablement de la qualité d'image sur l'ensemble des phases réalisées lors de l'acquisition dynamique.

En cas de mouvements involontaires répétés constatés, une correction de mouvement pouvait être opérée.

Après cette vérification, le choix de la VIF était réalisé. Dans notre étude, le mode de détection choisi était le mode automatique, préconisé pour l'exploration encéphalique. 
Ainsi, les vaisseaux (artères, veines et sinus veineux) étaient automatiquement détectés, et les meilleures entrées vasculaires étaient affichées.

Une fois la série dynamique traitée et l'entrée VIF sélectionnée, le logiciel était en mesure de calculer les cartographies.

Une fois, le calcul terminé, les cartographies étaient affichées, synchronisées et éventuellement fusionnées aux autres séquences réalisées. 


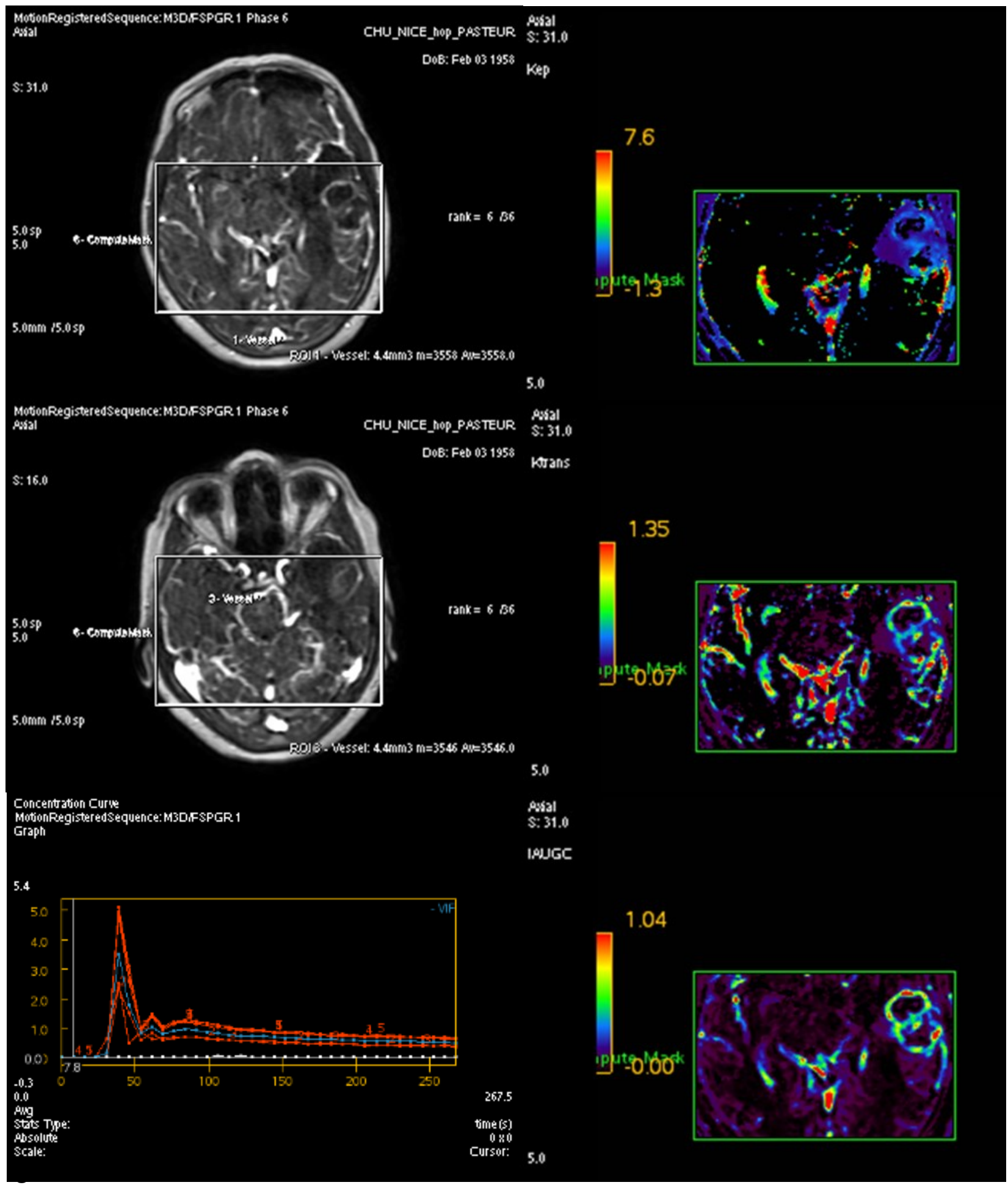

Figure 11 : Post-traitement des séquences de perfusion T1 à l'aide du logiciel GEN IQ

A gauche : mode de détection automatique de l'entrée vasculaire VIF

A droite : calcul des cartographies $k_{e p}, k_{\text {trans }}$ et IAUGC 


\subsubsection{Post-traitement en imagerie de perfusion $\mathrm{T} 2 *$}

Il a été réalisé à l'aide du mode « Brainstat AIF ».

Il est basé sur un algorithme prenant en compte une fonction d'entrée artérielle (signal en provenance d'une artère dans l'image).

Le choix de l'AIF peut être fait de façon globale ou locale.

Le choix d'une AIF globale doit se faire sur un gros vaisseau nourrissant tous les voxels du volume d'intérêt. Il est souvent choisi à la base du cerveau, par exemple dans l'une des artères cérébrales moyennes.

Le logiciel permettait le calcul de cartes paramétriques. 


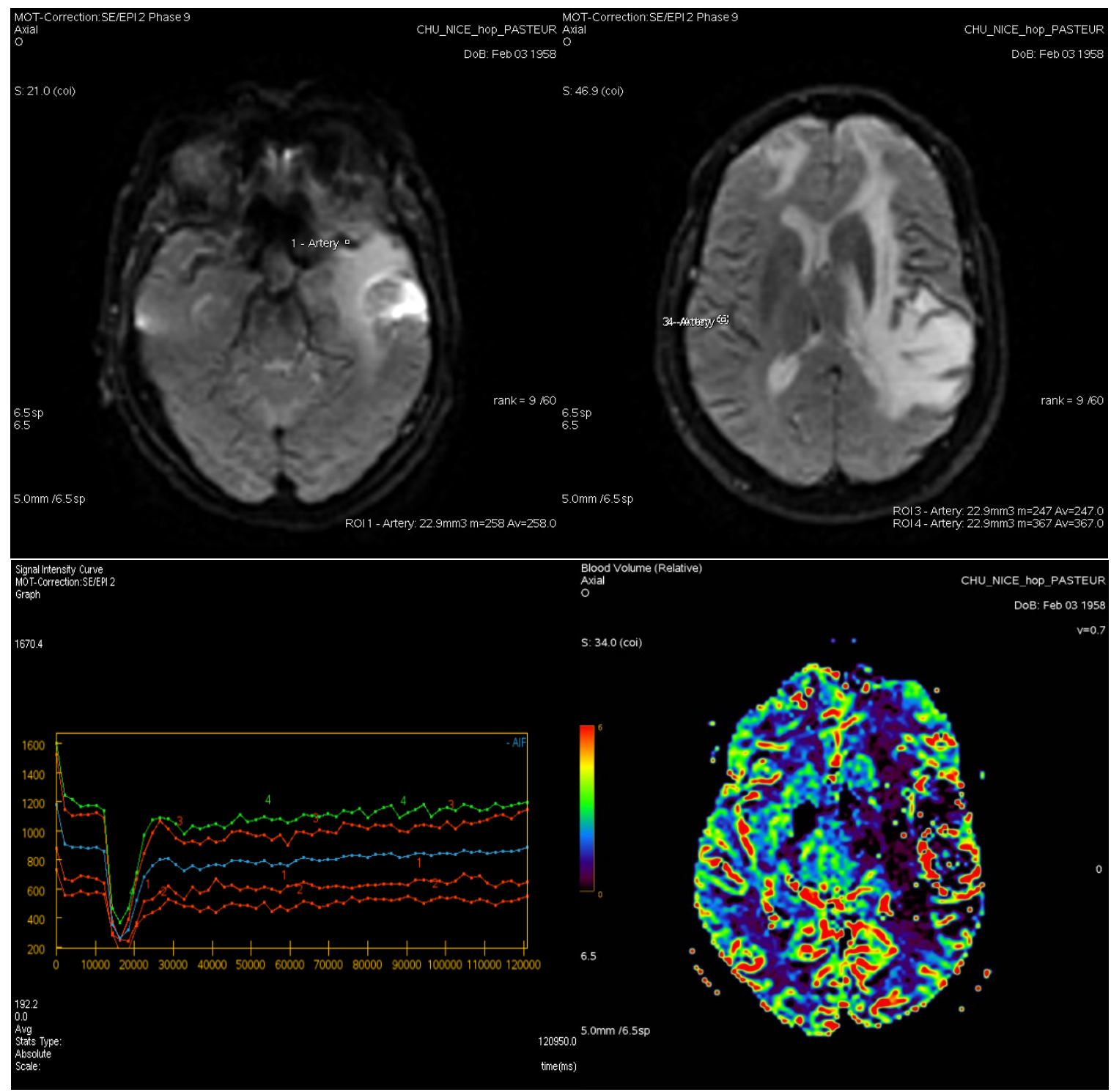

Figure 12 : Post-traitement en imagerie de perfusion T2* à l'aide du mode Brainstat AIF

En haut : mode de détection de l'entrée artérielle AIF

En bas, à droite : calcul de la cartographie de VSC 
3.2.2.3. Post-traitement en imagerie de diffusion

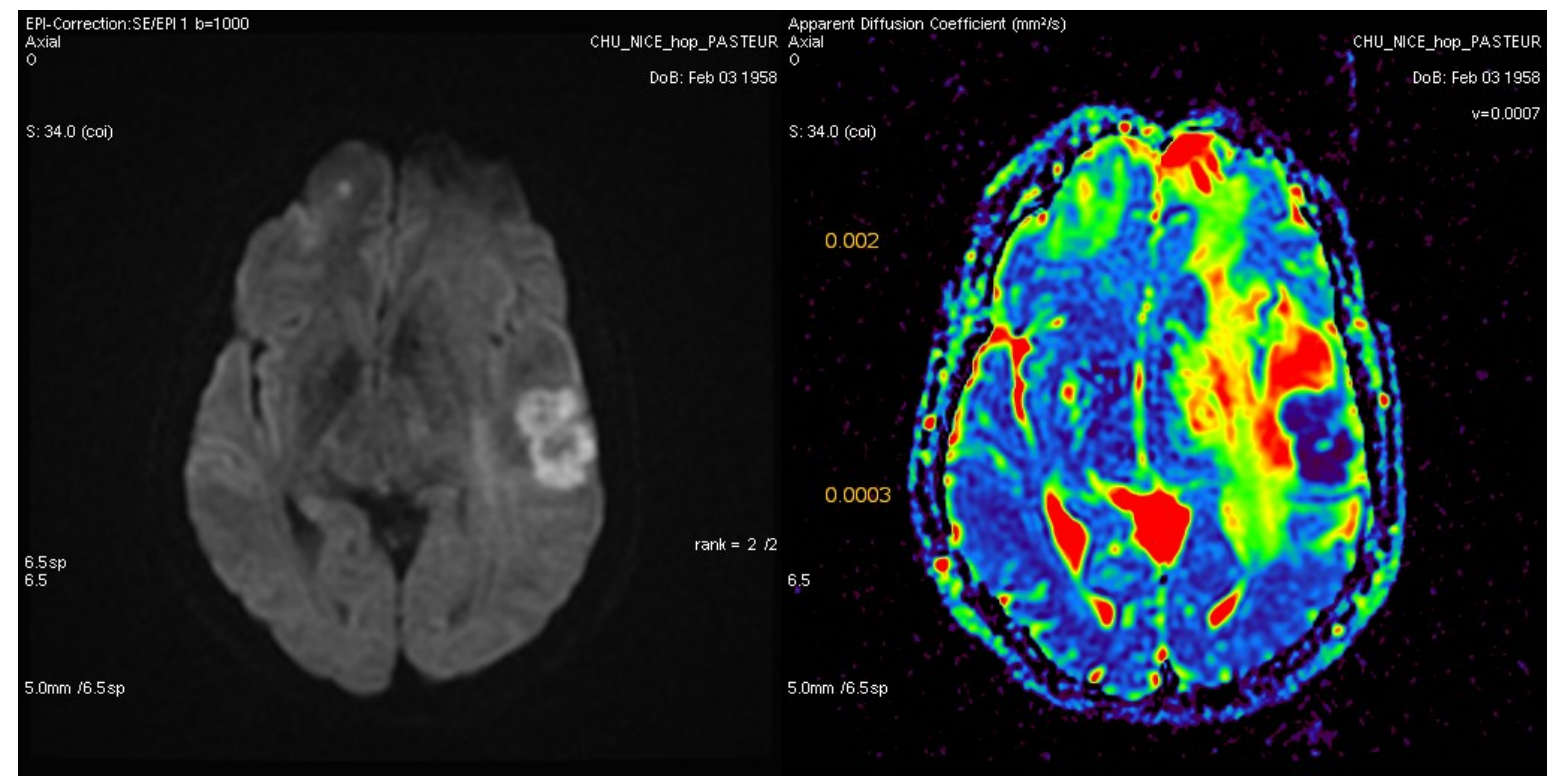

Figure 13 : Post-traitement en imagerie de diffusion

A gauche : séquence de diffusion

A droite : calcul de la cartographie ADC 


\subsection{Protocole de réalisation de la TEP-TDM à la ${ }^{18} \mathrm{~F}$-FDOPA}

Tous les patients ont bénéficié d'un examen par TEP-TDM à la ${ }^{18} \mathrm{~F}-\mathrm{FDOPA}$, réalisé dans le service de médecine nucléaire du Centre Antoine Lacassagne.

Conformément aux recommandations de la Société Française de Médecine Nucléaire (SFNM), une heure avant l'injection intraveineuse de ${ }^{18} \mathrm{~F}$-FDOPA (réalisée selon une posologie équivalente à $2 \mathrm{MBq} / \mathrm{kg}$ ), $100 \mathrm{mg}$ de carbidopa, inhibiteur de la DOPA décarboxylase périphérique, étaient administrés per os, afin d'augmenter d'un facteur 2, la biodisponibilité cérébrale de la ${ }^{18}$ F-FDOPA.

Vingt minutes après l'injection du radio-pharmaceutique, un topogramme de repérage, une tomodensitométrie (pour correction d'atténuation et repérage anatomique) ainsi que des acquisitions TEP centrées sur la région encéphalique (Biograph $\mathrm{mCT}$, Siemens Medical Systems, Erlanglen, Allemagne) étaient réalisés en mode statique pendant dix minutes.

Le patient se trouvait en décubitus dorsal, tête immobilisée dans une têtière, pour limiter les artéfacts cinétiques liés aux mouvements.

La reconstruction utilisait une méthode OSEM 5 itérations et 24 subsets avec correction d'atténuation et de diffusé, sans correction de résolution spatiale.

Le traitement des images incluait une fusion de la TEP à la ${ }^{18} \mathrm{~F}$-FDOPA à l'IRM cérébrale, afin d'optimiser l'analyse visuelle. 


\subsection{Données recueillies}

Les données ont été recueillies dans les dossiers médicaux et informatiques des patients inclus, grâce au logiciel Clinicom au CHU de Nice et au Centre Antoine Lacassagne (Centre de Lutte Contre le Cancer).

\subsubsection{Données épidémiologiques}

Les données recueillies concernant le patient étaient l'âge, le sexe et le caractère symptomatique de l’anomalie observée en imagerie.

Concernant la tumeur, les paramètres relevés concernaient la date et le type de prise en charge à savoir, biopsie stéréotaxique ou exérèse chirurgicale, fonction de la localisation anatomique et fonctionnelle de la lésion.

Le type histologique était aussi répertorié.

En cas de tumeur d'origine gliale, pour chaque patient, la présence d'une lésion de grade IV selon l'OMS et le statut MGMT étaient précisés.

En cas de métastase cérébrale, le type de tumeur primitive était rapporté. 


\subsubsection{Traitements associés}

Les paramètres recueillis concernant la radiothérapie étaient la date de fin de traitement, la dose totale délivrée et la présence d'un traitement antérieur par radiothérapie.

L'administration de chimiothérapie considérée à risque de radionécrose était prise en compte.

Enfin, l'administration d'un traitement anti-angiogénique par Bévacizumab était annotée.

\subsubsection{Données IRM}

Une analyse par double lecture, par un radiologue junior et un radiologue senior spécialisé en neuroradiologie, a été effectuée.

Le délai d'apparition de l'anomalie était répertorié par rapport à la date de fin du traitement par radiothérapie.

Les données recueillies étaient multiples.

\subsubsection{Données morphologiques}

Sur les séquences conventionnelles, 2 paramètres étaient relevés :

-la présence d’un rehaussement lésionnel était évaluée sur les séquences pondérées en T1 réalisées après injection de produit de contraste.

-en cas de rehaussement, le type de prise de contraste était alors précisé. 
Une analyse visuelle dichotomique permettait de distinguer un rehaussement nodulaire ou annulaire (figure 14) par opposition aux autres « patterns » de rehaussement connus (figure 15).
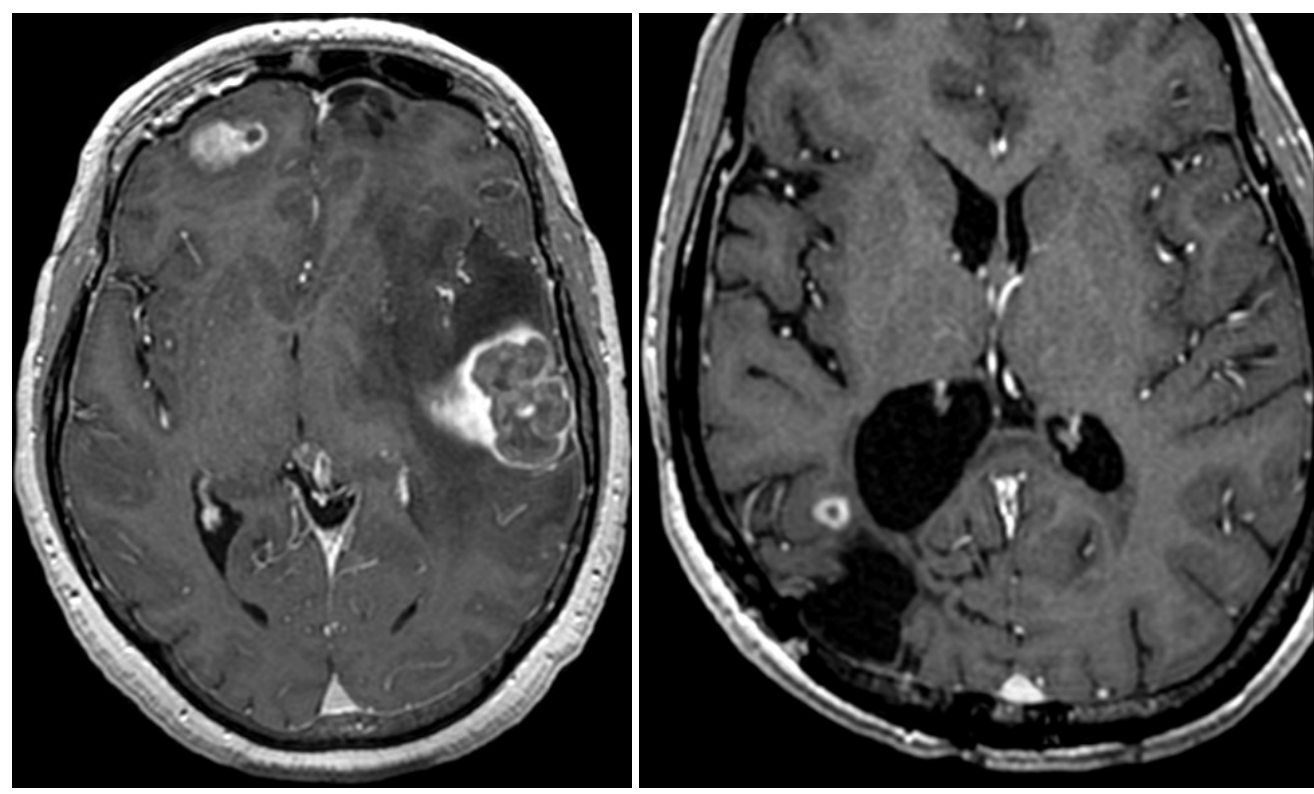

Figure 14 : Pattern de rehaussement de type nodulaire ou annulaire

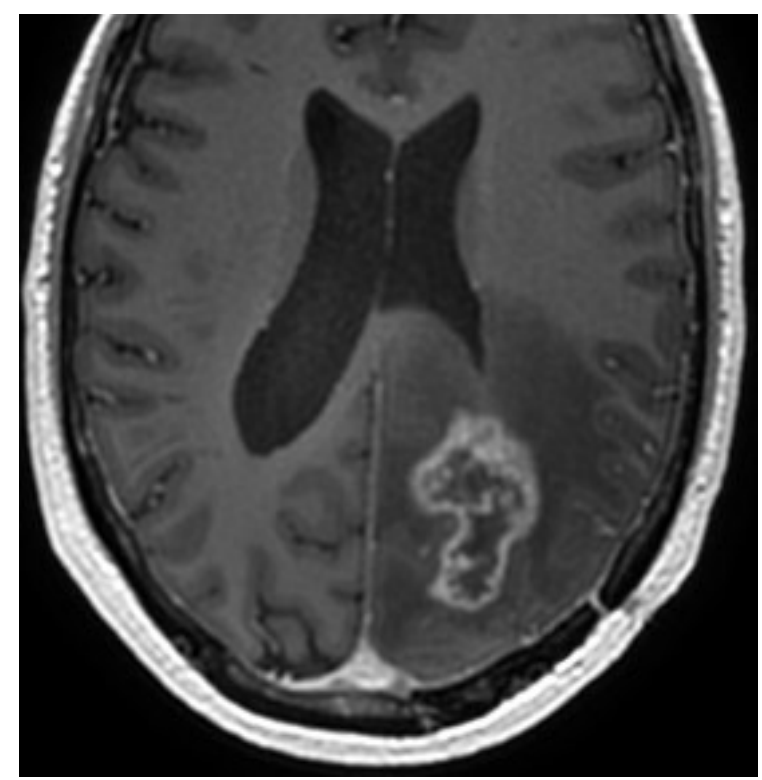

Figure 15 : Autres patterns de rehaussement 


\subsubsection{Données fonctionnelles}

Après réalisation de l'étape de post-traitement, elles étaient évaluées sur les séquences de diffusion et de perfusion.

Une région d'intérêt de $8 \times 8 \mathrm{~mm}$ était positionnée au sein de la prise de contraste pathologique, observée sur les séquences pondérées en T1 après injection de gadolinium.

Le logiciel utilisé permettait en utilisant l’option " générer ROI symétrique » de placer de manière automatique une région d'intérêt en zone saine, située dans l'hémisphère cérébral controlatéral. Celle-ci pouvait être modifiée manuellement en cas de positionnement erroné au sein de structures vasculaires, de sillons ou en région ventriculaire.

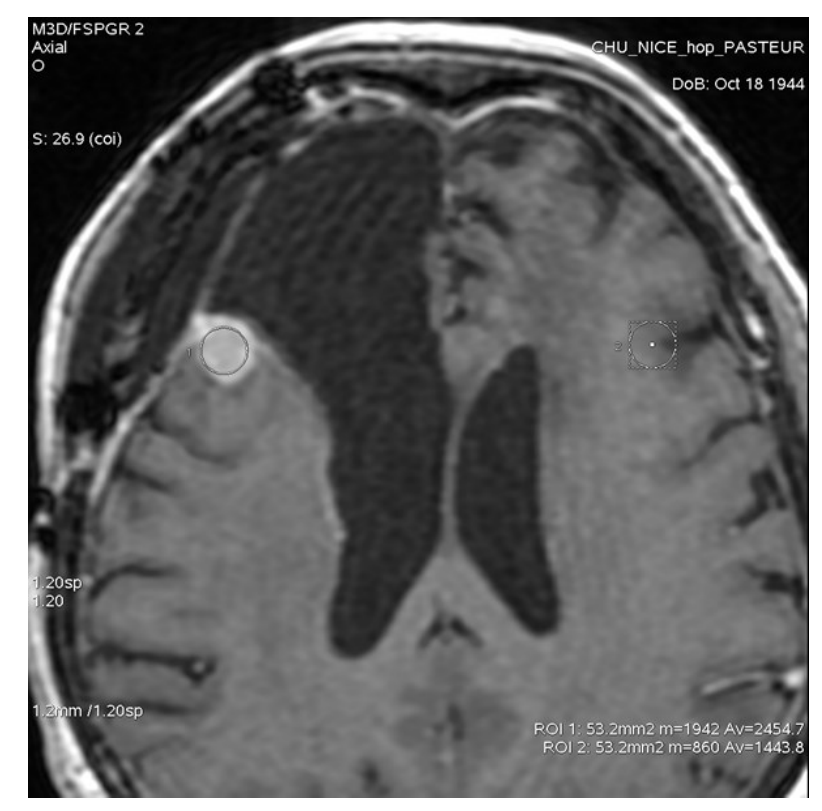

Figure 16: Positionnement d'une ROI au sein de la zone pathologique, avec ROI symétrique en zone saine controlatérale 
Une seconde option intitulée "propager à l'ensemble des séries " permettait la diffusion automatique de la région d'intérêt choisie à l'ensemble des séquences sélectionnées.

Données obtenues en imagerie de perfusion $T 2 *$

Sur la séquence de perfusion $\mathrm{T} 2 *, 3$ paramètres étaient relevés.

Sur la cartographie de volume sanguin cérébral, le paramètre $\mathbf{r}$ VSC était calculé à partir du rapport des valeurs de VSC obtenues en zone pathologique et zone saine controlatérale.
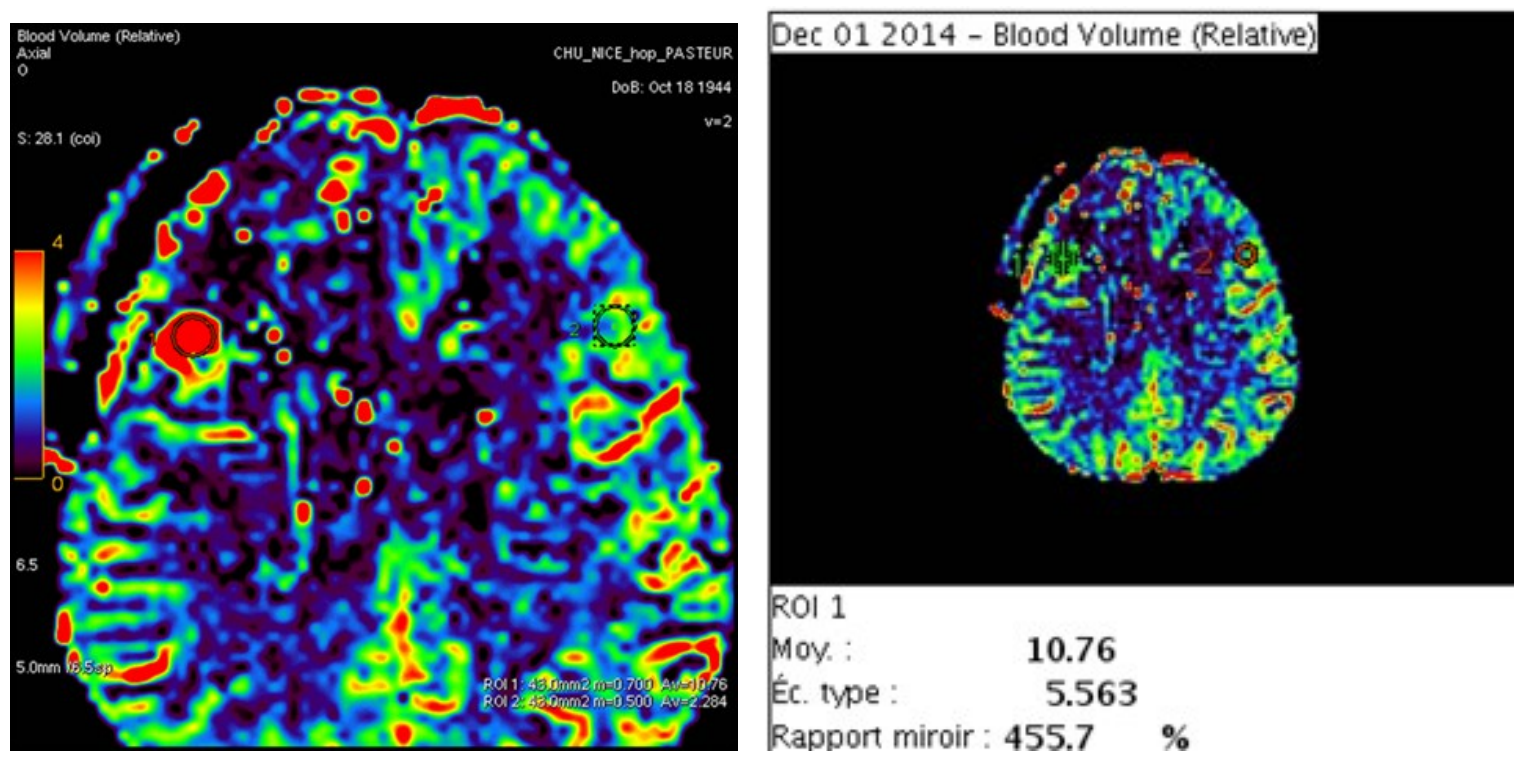

Figure 17 : Calcul du paramètre r VSC

En rouge : ROI positionnée en zone pathologique hyper-vascularisée

En vert : ROI positionnée en zone saine 
Les courbes obtenues lors du post-traitement de la séquence de perfusion T2* permettaient également le calcul des deux autres paramètres : $\boldsymbol{r}$ HP et PSR.

Le paramètre $\mathbf{r}$ HP était calculé à partir du rapport des valeurs de HP obtenues en zone pathologique et zone saine controlatérale.
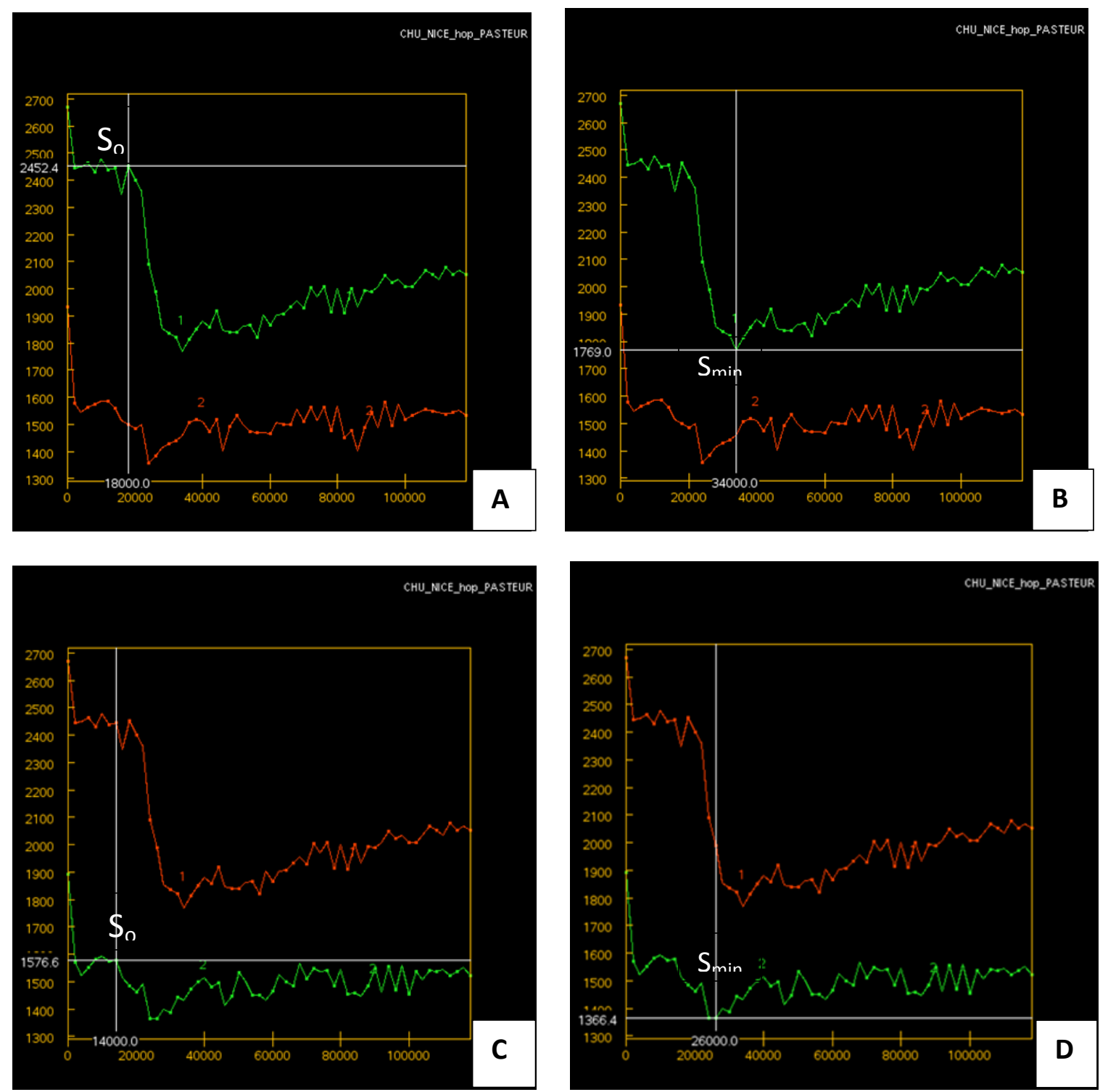

Figure 18 : Méthode de calcul du paramètre r HP

$\mathbf{H P}=\mathbf{S}_{0}-\mathbf{S}_{\min }$

$r H P=\left(S_{0}-S_{\min }\right)$ en zone pathologique/ $\left(S_{0}-S_{\min }\right)$ en zone saine

A et $B: S_{0}$ et $S_{\min }$ en zone pathologique

$C$ et $D: S_{0}$ et $S_{\min }$ en zone saine 
Le paramètre PSR était calculé à partir de l'équation $\mathbf{P S R}=\left(\mathbf{S}_{1}-\mathbf{S}_{\min }\right) /\left(\mathbf{S}_{0}-\mathbf{S}_{\min }\right)$ en zone pathologique.

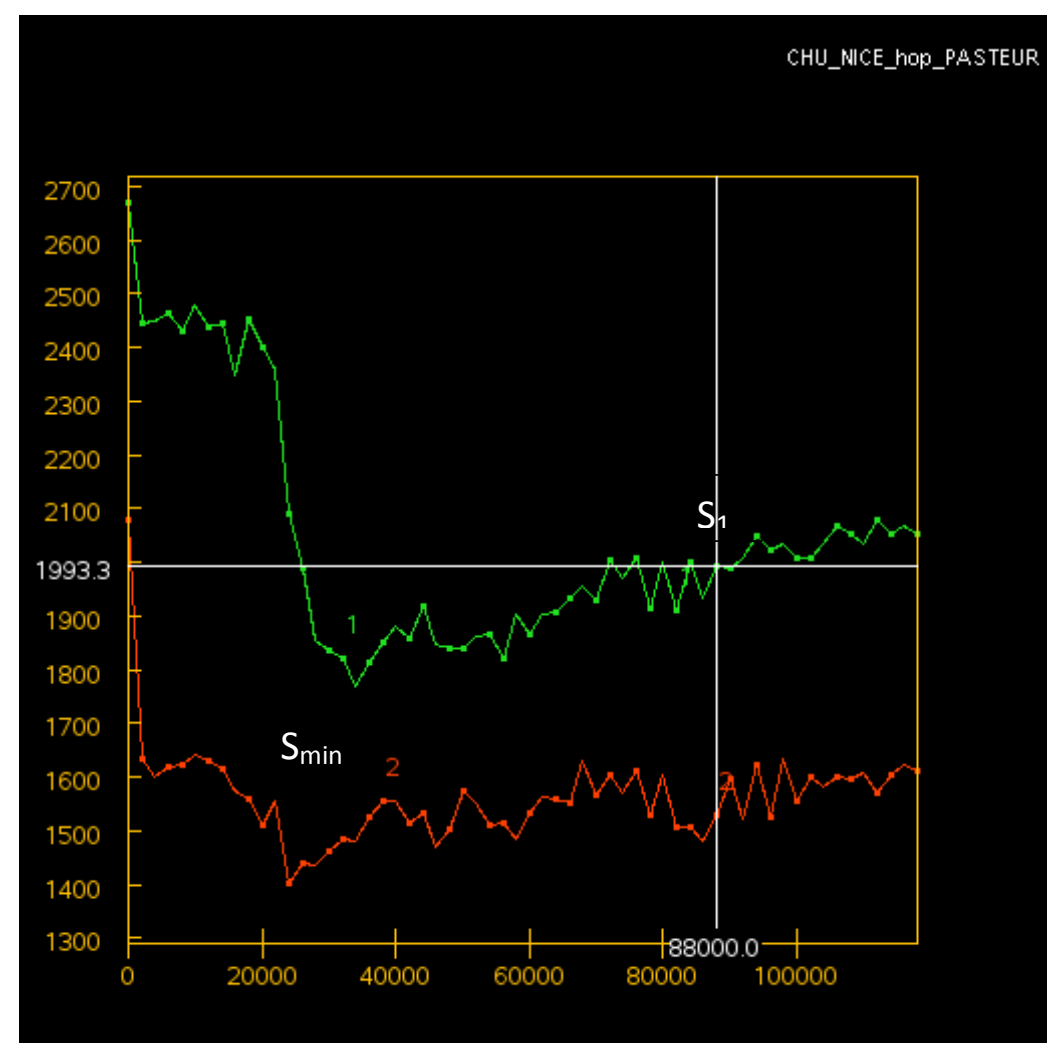

Figure 19 : Méthode de calcul du paramètre PSR

$\operatorname{PSR}=\left(\mathbf{S}_{1}-\mathbf{S}_{\min }\right) /\left(\mathbf{S}_{0}-\mathbf{S}_{\min }\right)$ 
Données obtenues en imagerie de perfusion T1

Sur la séquence de perfusion T1, 3 paramètres étaient relevés.

Sur les cartographies de $\mathbf{k}_{\text {trans, }}, \mathbf{k}_{\mathrm{ep}}$ et IAUGC, les paramètres rapport miroir, écart-type et moyenne étaient calculés automatiquement, à partir des valeurs obtenues en zone pathologique et zone saine controlatérale.
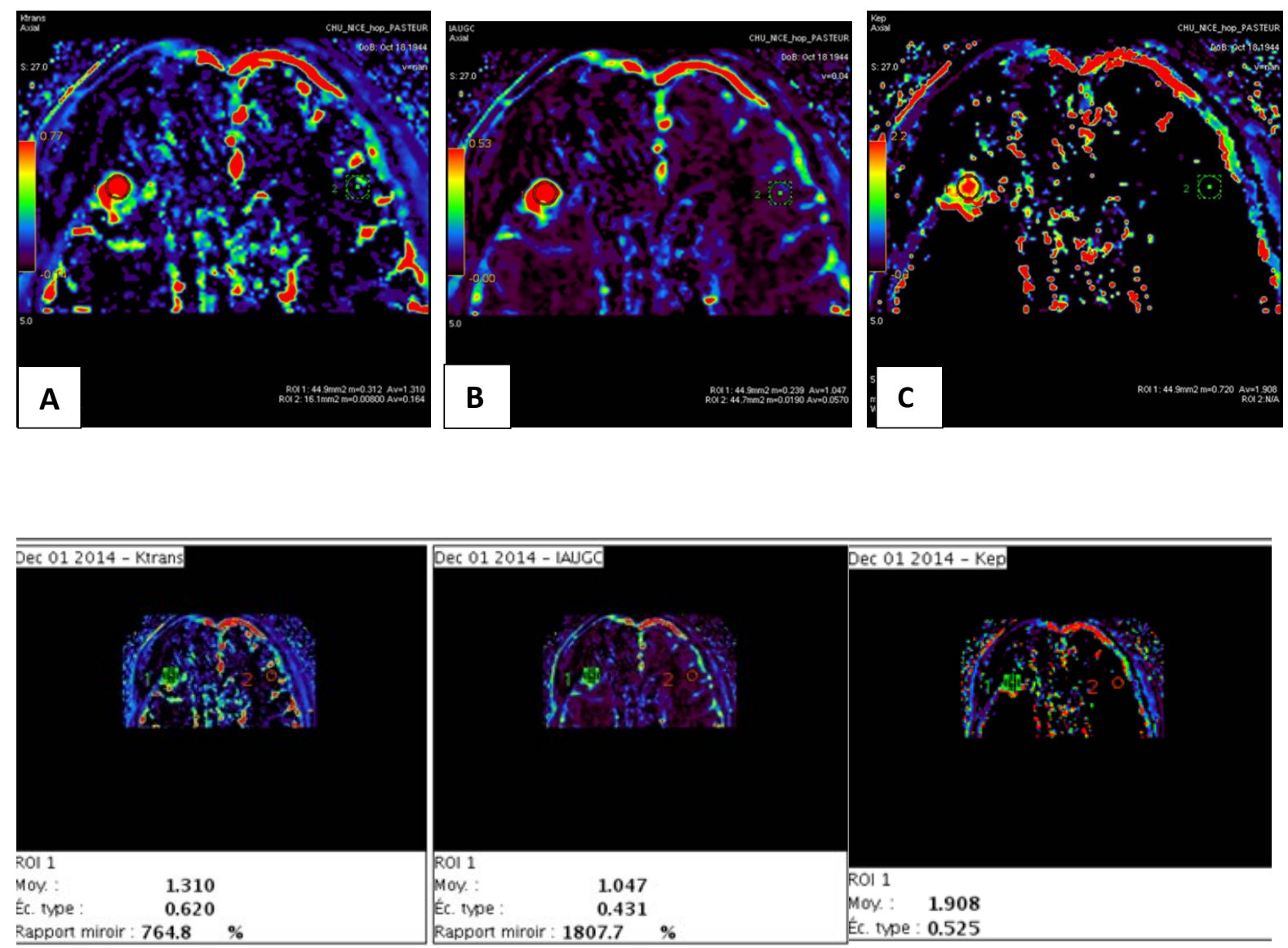

Figure 20 : Méthodes de calcul des paramètres $k_{\text {trans }}(A)$, IAUGC (B) et $K_{\text {ep }}(C)$

En rouge : ROI positionnée en zone pathologique

En vert : ROI positionnée en zone saine 
De plus, par analogie aux courbes de perfusion utilisées en IRM du sein et de la prostate notamment, le type de courbe en zone pathologique (figure 21).

La première partie de chaque courbe traduisait la présence d'agent de contraste dans le réseau capillaire.

La seconde partie de chaque courbe reflétait le rehaussement induit par la présence d'agent de contraste dans l'interstitium.

Trois profils distincts de rehaussement ont été définis dans notre étude, selon l'aspect de la seconde partie de la courbe.

-Le type 1 reflétait un passage progressif et continu de l'agent de contraste.

-Le type 2 correspondait à un passage avec stagnation de l'agent de contraste.

-Le type 3 traduisait un passage faible voire l'absence de passage de l'agent de contraste.
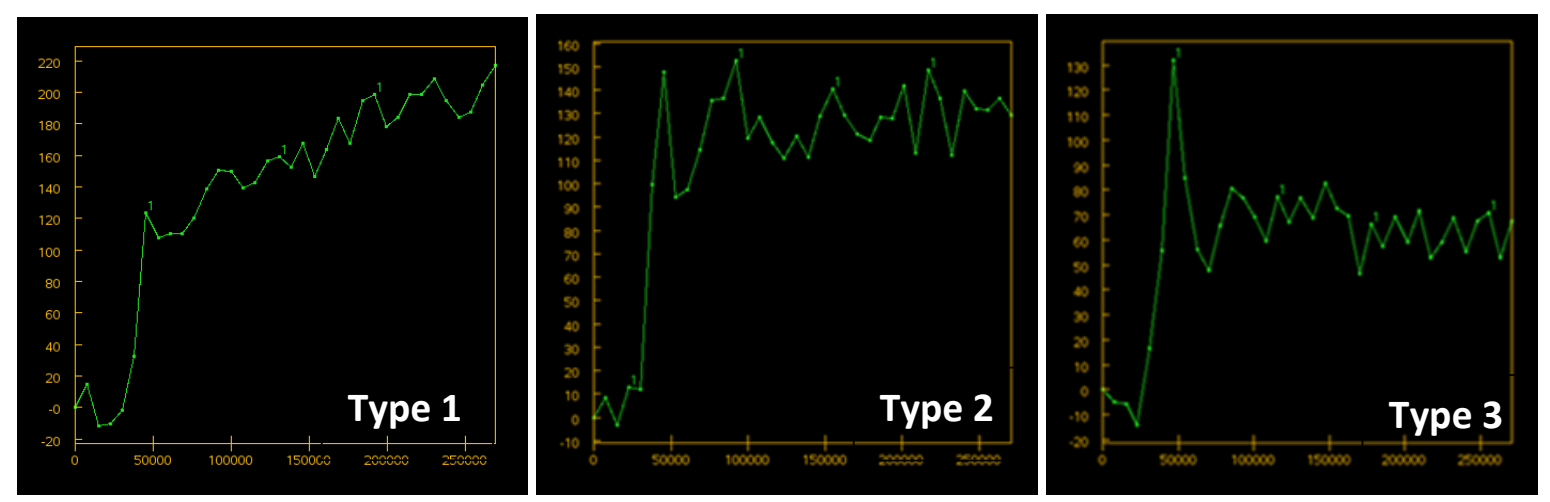

Figure 21 : Types de courbe de rehaussement en zone pathologique 
En zone saine, le profil de rehaussement correspondait à la courbe suivante (figure 22).

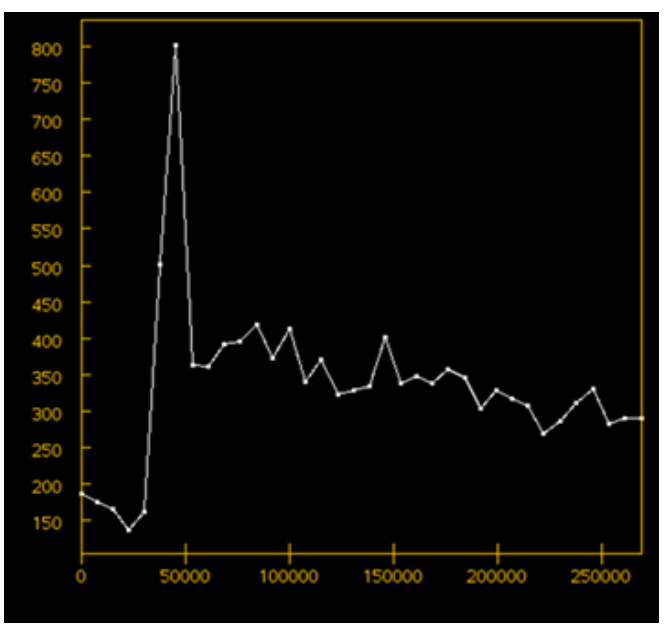

Figure 22 : Courbe de rehaussement en zone saine 
Données obtenues en imagerie de diffusion

Sur la cartographie d'ADC, le paramètre $r$ ADC était calculé à partir du rapport des valeurs $d^{\prime} A D C$ obtenues en zone pathologique et zone saine controlatérale.

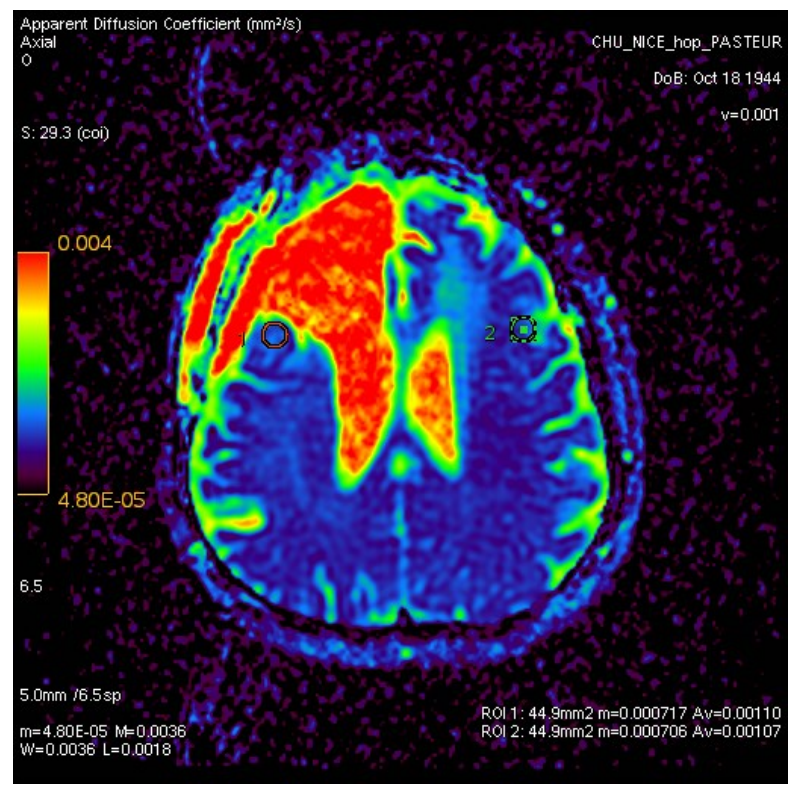

Figure 23 : Calcul du paramètre $r$ ADC

En rouge : ROI positionnée en zone pathologique

En vert : ROI positionnée en zone saine 


\subsubsection{Données métaboliques}

Obtenues par analyse de la spectroscopie, les données relevées après lecture en " mode Viewer » étaient d'ordre variable.

Une évaluation quantitative était réalisée par le biais de ratios calculés automatiquement parmi lesquels les ratios $\mathrm{Cho} / \mathrm{Cr}$ et NAA/Cr.

Une interprétation visuelle et qualitative du spectre de la lésion déterminait également la présence d'un pic de lipides et/ou de lactates et d'une élévation du pic de Choline, en l'absence de données quantitatives disponibles (figure 24).

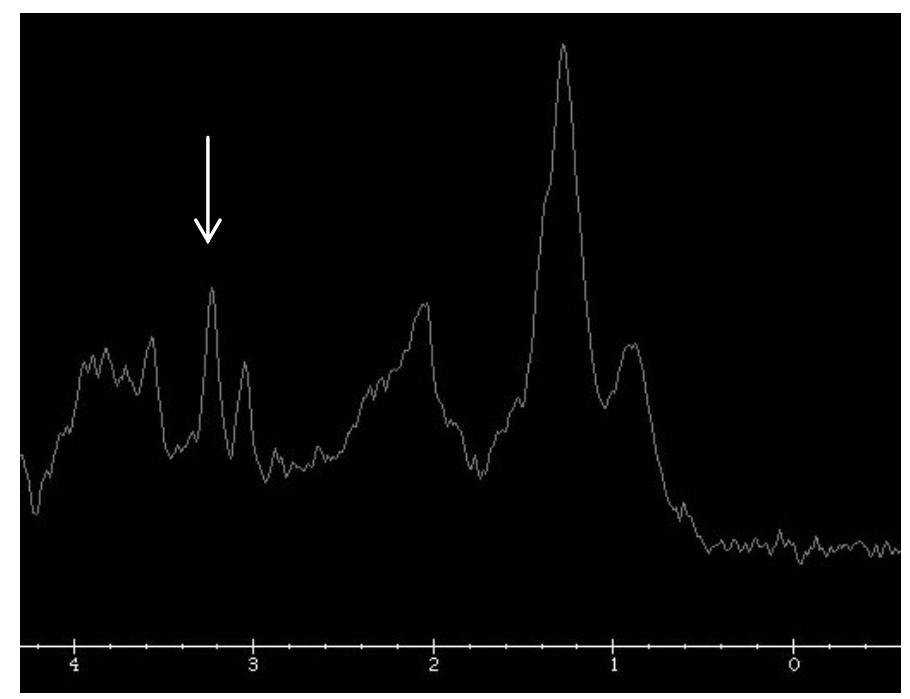

Figure 24: Spectre montrant la présence d'un pic de lipides et une élévation du pic de choline (flèche blanche).

Enfin, lorsque le spectre était ininterprétable compte-tenu d'artéfacts, cette donnée était aussi répertoriée. 
3.4.4. Données relatives à la TEP-TDM à la ${ }^{18}$ F-FDOPA

L'interprétation reposait essentiellement dans notre centre sur l'évaluation qualitative, selon l'échelle visuelle, score côté de 0 à 3, dépendant de l'intensité de fixation de la lésion par rapport aux striata, comme rappelé précédemment.

Le diagnostic de récidive était ainsi porté lorsque l'intensité de fixation lésionnelle était supérieure ou égale à la fixation des striata, soit une échelle visuelle supérieure ou égale à 2 (figure 25).

La radionécrose était par conséquent évoquée en cas de score égal à 0 ou 1 (figure 25). 

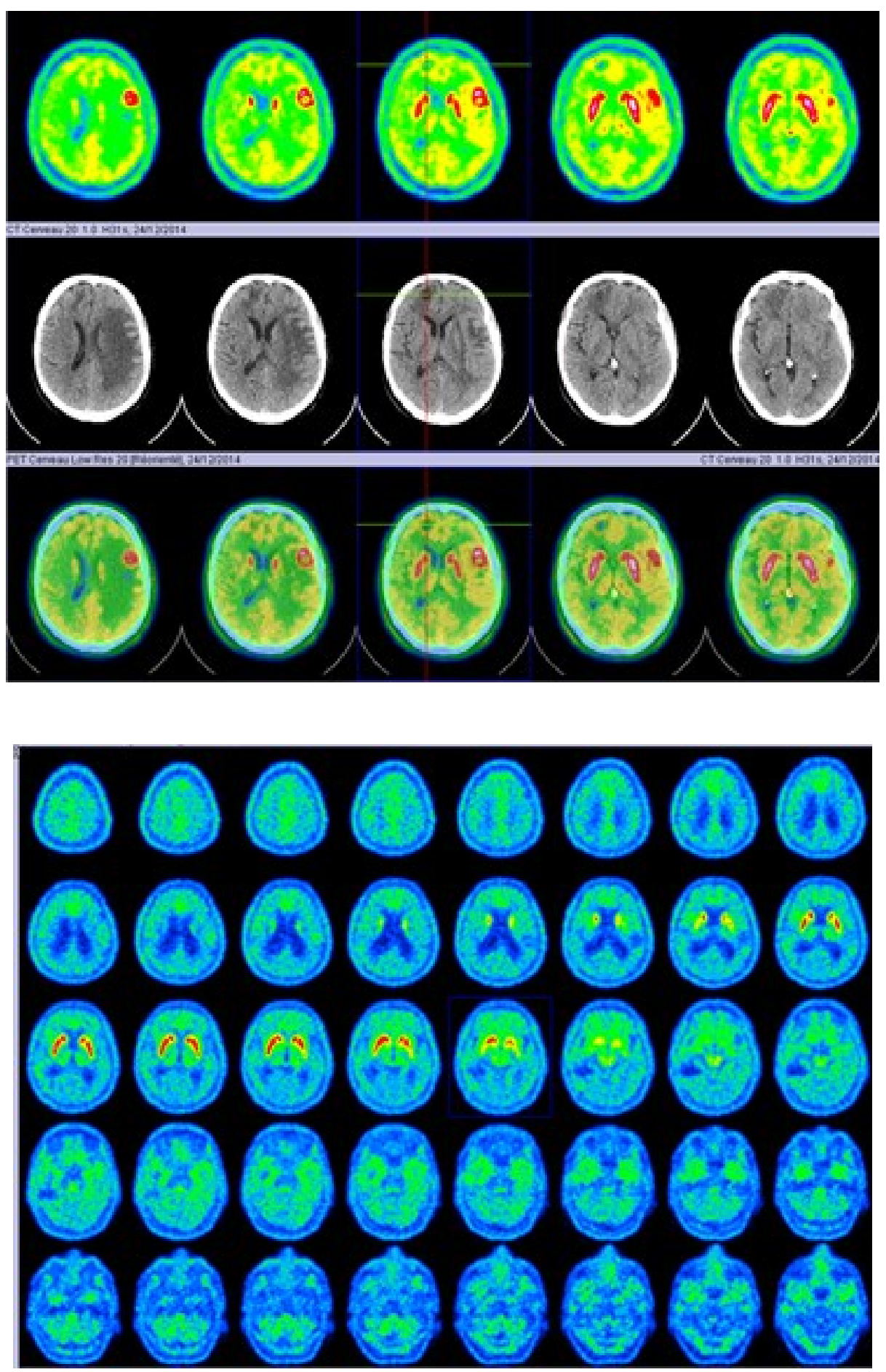

Figure 25 : TEP-TDM à la ${ }^{18}$ F-FDOPA

En haut: intensité de fixation d'une lésion pariétale gauche supérieure à celle des striata (échelle visuelle=3)

En bas: absence de fixation du radio-pharmaceutique (échelle visuelle=0) 


\subsection{Gold standard}

Le gold-standard pouvait être porté par diagnostic anatomo-pathologique, lorsqu'une nouvelle biopsie ou chirurgie d'exérèse avait pu être réalisée.

Dans le cas contraire, un suivi clinico-radiologique, conformément aux données de la littérature, selon un délai variable allant de 3 à 12 mois, servait de référence.

Ainsi, l'apparition d'une prise de contraste ou d'une nouvelle lésion, se majorant sur l'imagerie de suivi faisait porter le diagnostic de progression tumorale.

A l'inverse, la stabilité ou la diminution de l'anomalie observée en imagerie, sans modification du schéma thérapeutique, orientait vers le diagnostic de radionécrose.

\subsection{Critères de jugement}

Le critère principal de jugement était le diagnostic porté de radionécrose ou de récidive tumorale, grâce à la combinaison d'outils qualitatifs et quantitatifs en IRM multimodale, en comparaison au gold-standard disponible (histologique ou par suivi clinico-radiologique).

Le critère secondaire de jugement était l'appréciation de la concordance des résultats obtenus par IRM multimodale et TEP-TDM à la ${ }^{18} \mathrm{~F}$-FDOPA. 


\subsection{Analyses statistiques}

Les données quantitatives ont été représentées sous forme de médiane, moyenne et écarttype, concernant l'analyse univariée.

Le test $\mathrm{du} \mathrm{Chi}^{2}$ ou le test exact de Fisher lorsque les conditions d'application du $\mathrm{CHi}^{2}$ n'étaient pas satisfaites a été utilisé pour comparer les variables qualitatives.

Le test de Kruskal-Wallis a été utilisé pour comparer les variables qualitatives et quantitatives compte tenu de la distribution non normale des données.

Le calcul du coefficient kappa a été réalisé pour évaluer la concordance des données qualitatives. La méthode de Bland et Altman a été effectuée pour apprécier la concordance des données quantitatives.

Le calcul des seuils décisionnels $\mathrm{N}$ (radionécrose) versus $\mathrm{P}$ (progression tumorale) a utilisé l'analyse discriminante linéaire.

Le degré de significativité était fixé pour une valeur de $p$ inférieure à 0,05.

\subsection{Recherche bibliographique}

La recherche bibliographique a été effectuée en utilisant la base de données Pubmed.

Les mots clés utilisés étaient : brain tumors, cerebral metastasis, glioblastoma, conventional MR imaging, dynamic susceptibility contrast MRI, dynamic contrast enhanced MRI, perfusion, diffusion, radionecrosis, pet imaging. 


\section{4 - RESULTATS}

\subsection{Population}

\subsubsection{Données démographiques}

De janvier 2013 à juillet 2015, 61 patients, répartis en 34 hommes (52\%) et 27 femmes (48\%), âgés en moyenne de $59 \pm 11,5$ ans (min 36 ans; max 89 ans) ont bénéficié d'un protocole standardisé d'IRM multimodale dans le service d'imagerie de l'Hôpital Pasteur, réalisé pour doute entre radionécrose et récidive tumorale, lors du suivi de tumeurs cérébrales irradiées.

\subsubsection{Type histologique}

Les tumeurs cérébrales étaient réparties en tumeurs gliales $(n=35)$ et métastases cérébrales $(n=26)$.

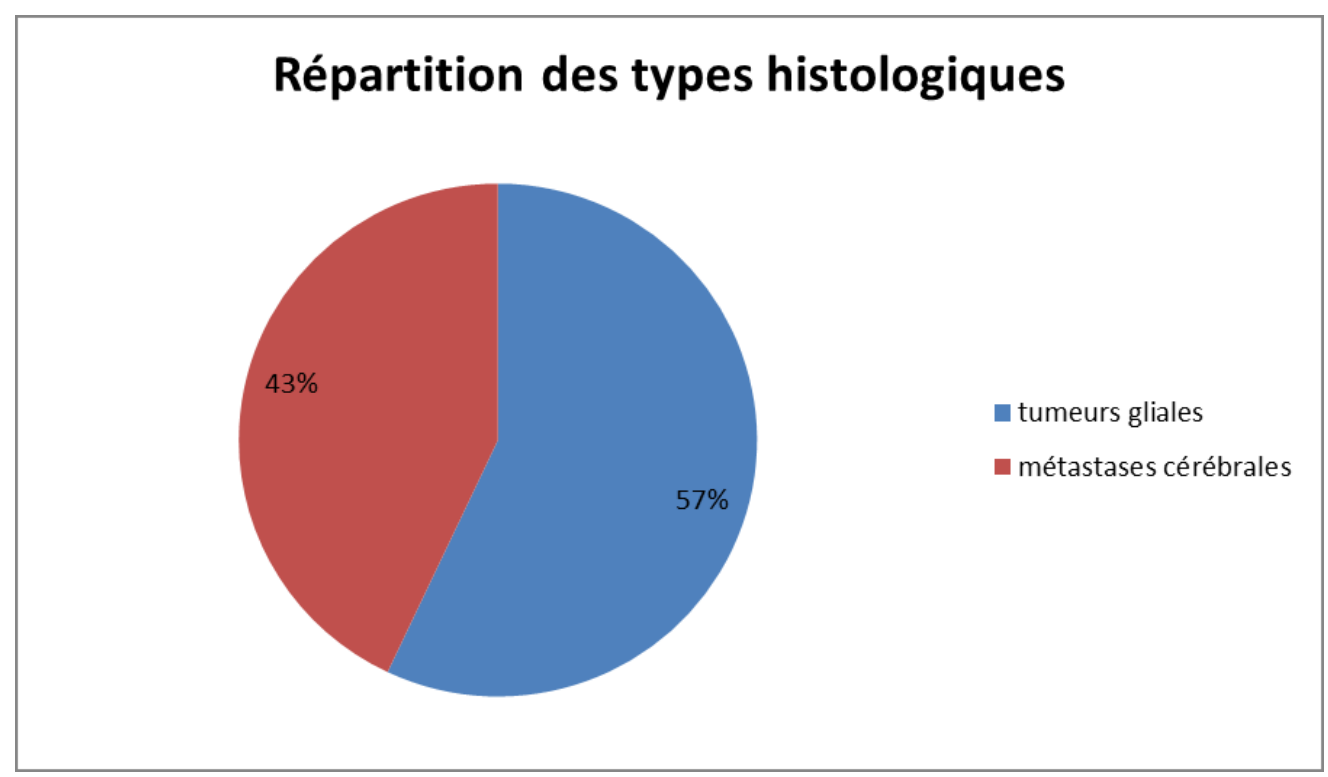

Figure 26 : Répartition des types histologiques dans la population globale 
Les tumeurs gliales étaient réparties en tumeurs de bas grade $(n=6)$ et de haut grade $(n=29)$.

Les métastases cérébrales étaient réparties selon le type de primitif : pulmonaire $(n=13)$, mammaire $(n=5)$, digestif $(n=2)$, oro-pharyngé $(n=2)$, rénal $(n=2)$ ou secondaire à un mélanome $(n=2)$.

\section{Répartition des métastases cérébrales}

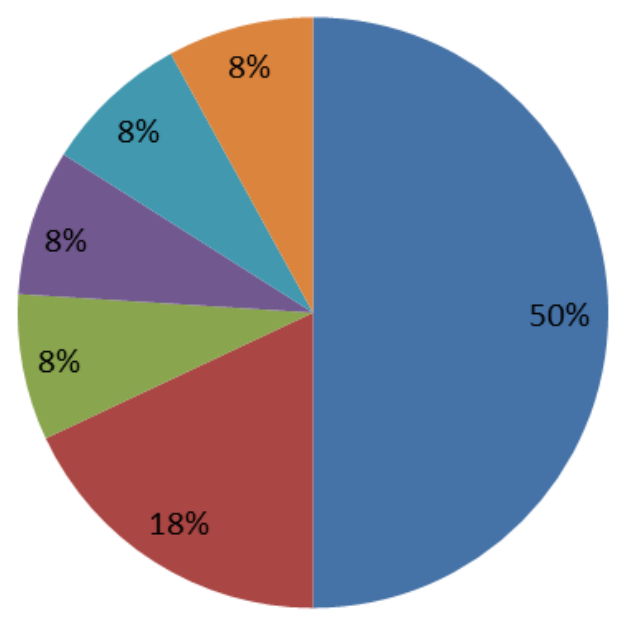

- origine pulmonaire

匹 origine mammaire

origine oro-pharyngée

origine digestive

- origine rénale

mélanome

\section{Répartition des tumeurs gliales}

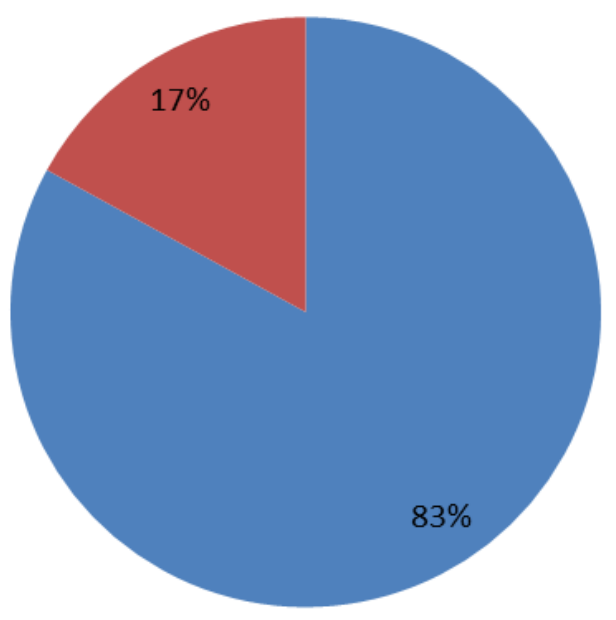

- tumeurs gliales de haut grade

- tumeurs gliales de bas grade

Figure 27 : Répartition des tumeurs gliales et des métastases cérébrales dans la population 


\subsubsection{Modalités du diagnostic final}

Les patients étaient classés en deux groupes respectivement appelés $P$ en cas de progression tumorale et $\mathrm{N}$ en cas de radionécrose.

Le gold-standard utilisé pour déterminer le groupe d'appartenance était porté sur des données histologiques ( $n=13$ ) ou grâce au suivi clinico-biologique $(n=48)$.

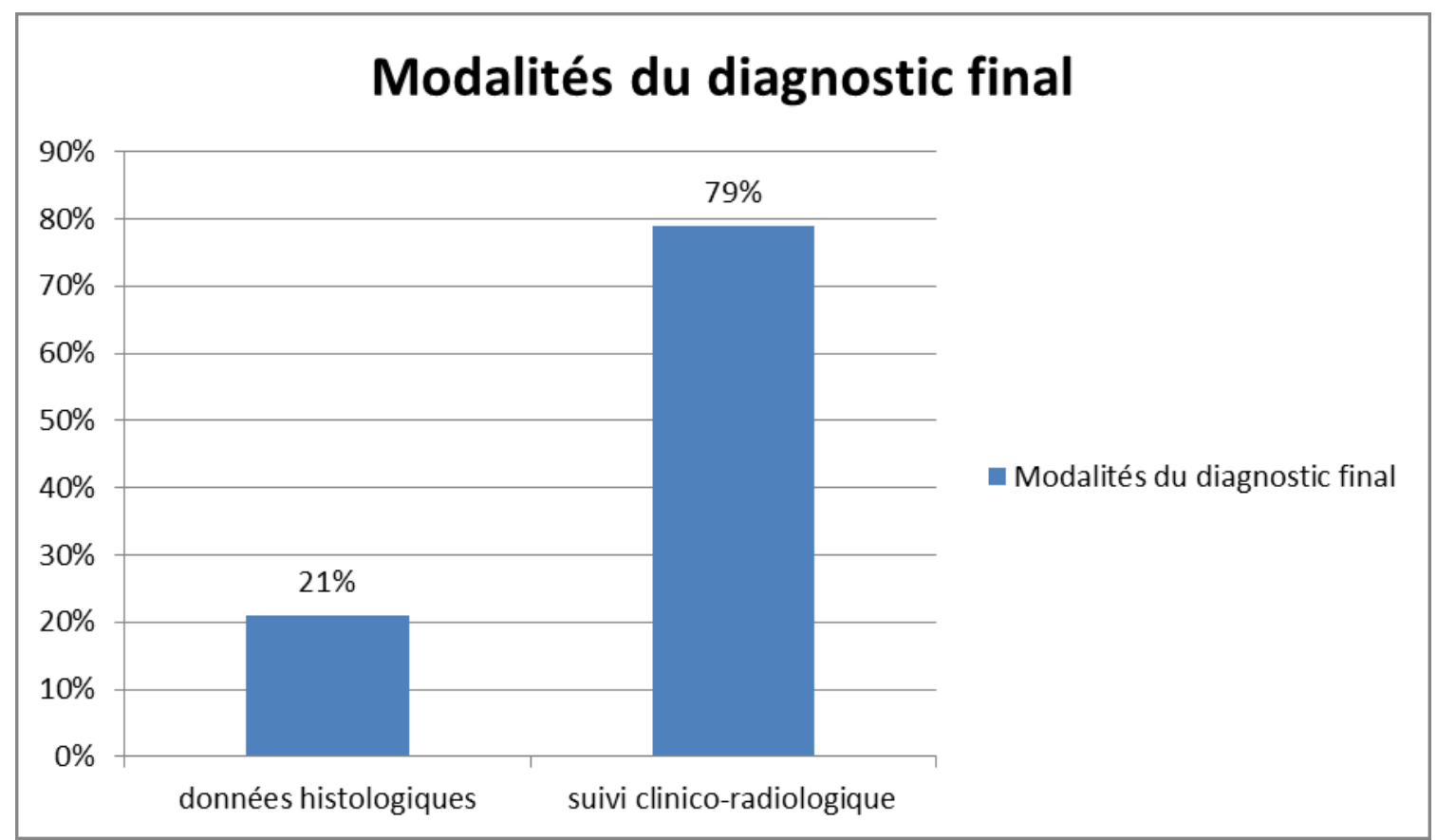

\section{Figure 28 : Modalités du diagnostic final}

La combinaison des deux modalités a permis de diagnostiquer sur la population globale, $48 \%$ $(n=29)$ de patients appartenant au groupe $P$ et $52 \%(n=32)$ au sein du groupe $N$. 


\subsubsection{Traitements associés}

\subsubsection{Radiothérapie}

Le délai moyen d'apparition d'une anomalie en IRM après irradiation était de 351 jours, avec une médiane de 245 jours.

L'analyse bi-variée au seuil de $5 \%$ n'a pas montré de différence significative concernant la dose totale d'irradiation reçue $(p=0,736)$, l'antécédent d'irradiation cérébrale $(p=0,767)$ et l'administration d'une chimiothérapie considérée à risque de radionécrose $(p=0,202)$ entre les patients présentant une radionécrose et ceux atteints de récidive tumorale.

\subsubsection{Traitement anti-angiogénique}

La prise d'un traitement anti-angiogénique par Bévacizumab n'influait pas significativement entre les deux groupes $\mathrm{P}$ et $\mathrm{N}(p=1)$. 


\begin{tabular}{|c|c|}
\hline Caractéristiques & Nombre \\
\hline \multicolumn{2}{|c|}{ Age (années) } \\
\hline Moyen & $59 \pm 11,5$ \\
\hline Rang & $36-89$ \\
\hline \multicolumn{2}{|c|}{ Sexe } \\
\hline Masculin & 34 \\
\hline féminin & 27 \\
\hline Histologie Gliomes & 35 \\
\hline Bas grade & 6 \\
\hline Haut grade & 29 \\
\hline Histologie Métastases & 26 \\
\hline \multicolumn{2}{|c|}{ Type de résection chirurgicale } \\
\hline Exérèse totale & 44 \\
\hline Biopsie Stéréotaxique & 6 \\
\hline \multicolumn{2}{|c|}{$\begin{array}{ll}\text { Radiothérapie } \\
\end{array}$} \\
\hline Antécédent d'irradiation & 15 \\
\hline Dose d'irradiation (Gy) : rang & $12-124$ \\
\hline \multicolumn{2}{|c|}{ Chimiothérapie } \\
\hline A risque de radionécrose & 32 \\
\hline Traitement par Bévacizumab & 10 \\
\hline Anomalie à l'examen clinique & 25 \\
\hline
\end{tabular}

Tableau 1 : Caractéristiques de la population 


\subsection{Interprétation de l'IRM multimodale}

Les résultats ont été calculés sur les données reportées par le radiologue junior.

L'interprétation de l'IRM multimodale, basée sur l'ensemble des séquences réalisées, a été comparée au gold-standard.

Dans notre étude, $8 \%(n=5)$ des cas étaient discordants.

La concordance entre l'interprétation de l'IRM multimodale et le gold-standard a été analysée à l'aide du calcul du coefficient Kappa de Cohen.

Selon la valeur du coefficient Kappa, la concordance était variable : médiocre lorsqu'il était $\leq 0,40$, modéré s'il était compris entre 0,41 et 0,60 , bon s'il était compris entre 0,61 et 0,80 et excellent au-delà de 0,81.

Les valeurs de sensibilité, spécificité, valeurs prédictives positive (VPP) et négative (VPN), les rapports de vraisemblance (RV) positifs et négatifs, ainsi que le coefficient kappa de Cohen concernant l'interprétation de l'IRM multimodale par rapport au gold-standard considéré, étaient calculées et résumées dans le tableau 2. 


\begin{tabular}{|c|c|c|}
\hline Paramètres & \multicolumn{2}{|c|}{ Estimation } \\
\hline \multirow{2}{*}{ Sensibilité } & $96,60 \%$ & \\
\hline & \multicolumn{2}{|c|}{ IC $95 \%=82,8 \%-99,4 \%$} \\
\hline \multirow{2}{*}{ Spécificité } & \multicolumn{2}{|c|}{$87,50 \%$} \\
\hline & \multicolumn{2}{|c|}{ IC $95 \%=71,9 \%-95,0 \%$} \\
\hline \multirow{2}{*}{ VPP } & \multicolumn{2}{|c|}{$87,50 \%$} \\
\hline & \multicolumn{2}{|c|}{ IC $95 \%=71,9 \%-95 \%$} \\
\hline \multirow{2}{*}{ VPN } & \multicolumn{2}{|c|}{$96,60 \%$} \\
\hline & \multicolumn{2}{|c|}{ IC $95 \%=82,8 \%-99,4 \%$} \\
\hline \multirow{2}{*}{ RV+ } & \begin{tabular}{|r|}
7,72 \\
\end{tabular} & \\
\hline & \multicolumn{2}{|c|}{ IC $95 \%=4,7-12,6$} \\
\hline \multirow{2}{*}{ RV- } & 0,04 & \\
\hline & \multicolumn{2}{|c|}{ IC $95 \%=0,005-0,28$} \\
\hline \multirow{2}{*}{ Coefficient Kappa } & 0,84 & \\
\hline & \multicolumn{2}{|c|}{ IC $95 \%=0,59-1,09$} \\
\hline
\end{tabular}

Tableau 2 : Concordance et performances diagnostiques de l'IRM multimodale (lecture par le radiologue junior) par rapport au gold-standard. 


\subsubsection{Paramètres morphologiques}

La présence d'un rehaussement lésionnel, rapportée chez $82 \%$ des patients ( $n=50)$, était significativement différente, préférentiellement observée en cas de progression tumorale.

En revanche, le pattern de rehaussement n'était pas discriminant entre les deux groupes.

\begin{tabular}{|cccc|}
\hline & $\mathbf{P}$ & $\mathbf{N}$ & $\boldsymbol{P}$ \\
\hline Rehaussement lésionnel & $28(56 \%)$ & $22(44 \%)$ & 0,018 \\
\hline Type de rehaussement & & & 1 \\
Nodulaire & $11(22 \%)$ & $7(14 \%)$ & \\
Autres types & $19(38 \%)$ & $13(26 \%)$ & \\
\hline
\end{tabular}

Tableau 3 : Données morphologiques 


\subsubsection{Paramètres fonctionnels}

4.2.2.1. Sur les séquences de perfusion $\mathrm{T} 2 *$

Les données quantitatives calculées à partir des séquences de perfusion T2* étaient résumées par le tableau 4 et la figure 29.

\begin{tabular}{|c|c|c|c|}
\hline & $P(n=29)$ & $N(n=32)$ & $P$ \\
\hline rVSC & & & $<0,001^{*}$ \\
\hline Moyenne \pm écart-type & $3,30 \pm 3,26$ & $1,02 \pm 0,90$ & \\
\hline Médiane (Min-Max) & $2,26(0,56-6,85)$ & $0,72(0,34-4,9)$ & \\
\hline$r$ HP & & & $0,001^{*}$ \\
\hline Moyenne \pm écart-type & $2,21 \pm 3,07$ & $0,92 \pm 0,55$ & \\
\hline Médiane (Min-Max) & $1,47(0,49-6,71)$ & $0,74(0,14-2,16)$ & \\
\hline PSR & & & $<0,001^{*}$ \\
\hline Moyenne \pm écart-type & $0,76 \pm 0,33$ & $1,19 \pm 0,57$ & \\
\hline Médiane (Min-Max) & $0,73(0,27-1,72)$ & $1,10(0,10-2,8)$ & \\
\hline
\end{tabular}

*test de Kruskal-Wallis

Tableau 4 : Mesures de paramètres quantitatifs ( $r$ VSC, $r$ HP et PSR) par imagerie de perfusion T2* dans les deux groupes. 


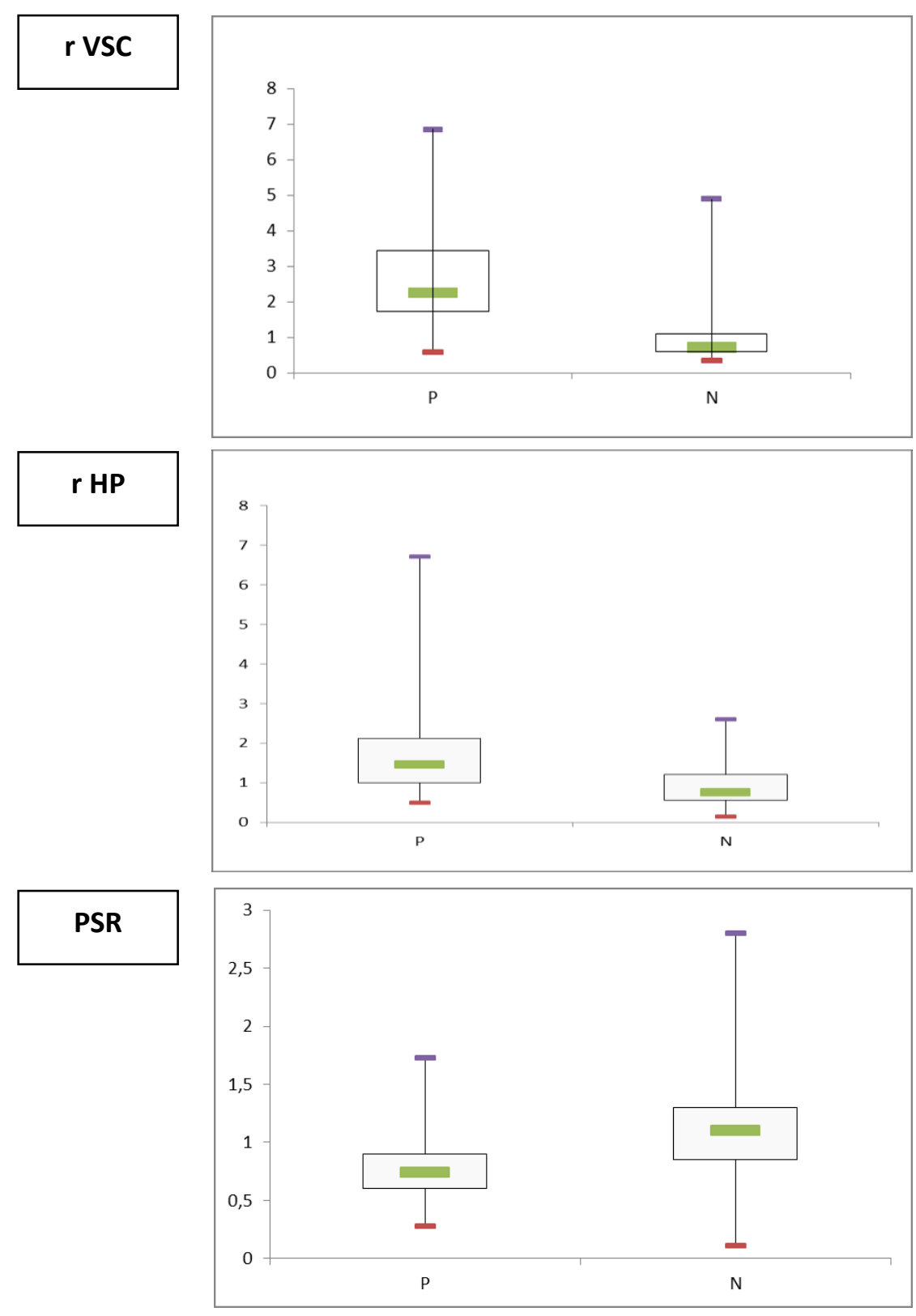

Figure 29: Représentation des paramètres mesurés en imagerie de perfusion T2* sous forme de boîtes à moustache.

Par convention, le trait vert représente la valeur médiane. Le bas et le haut de la boîte représentent respectivement le premier et le troisième quartile. Les autres segments correspondent aux valeurs extrêmes.

P et $\mathbf{N}$ signifient Progression tumorale et Radionécrose. 
Les valeurs mesurées de $r$ VSC et de r HP étaient significativement plus élevées dans le groupe $\mathrm{P}$ par rapport à celles du groupe $\mathrm{N}$.

L'influence d'un traitement anti-angiogénique sur le calcul du r VSC a été analysée.

$20 \%(n=12)$ des patients recevaient un traitement par Bevacizumab au moment de I'IRM multimodale réalisée. Les valeurs de $r$ VSC calculées chez les patients sous traitement antiangiogénique étaient moins élevées, avec une médiane de 0,76 versus 1,51 en l'absence de traitement, sans différence statistiquement significative $(p=0,485)$.

Les valeurs mesurées de PSR étaient, de façon statistiquement significative, inférieures en cas de progression tumorale.

L'analyse discriminante linéaire complémentaire a permis de déterminer des seuils décisionnels pour chacun de ces paramètres pour prédire le statut $\mathrm{P}$ ou $\mathrm{N}$.

Le meilleur classement obtenu était de 75,4\% avec un seuil de $r$ VSC=2,2.

Les meilleurs classements obtenus étaient respectivement de $65,4 \%$ et $72,1 \%$ avec des seuils de $r \mathrm{HP}=1,6$ et de $\mathrm{PSR}=1$. 


\subsubsection{Sur les séquences de perfusion $\mathrm{T} 1$}

Les données quantitatives calculées à partir des séquences de perfusion T1 étaient résumées par le tableau 5 et la figure 30.

\begin{tabular}{|c|c|c|c|}
\hline & $\mathbf{P}$ & $\mathbf{N}$ & $\boldsymbol{P}$ \\
\hline $\mathbf{k}_{\text {trans }}$ moyen & & & $0,001^{*}$ \\
\hline Moyenne \pm écart-type & $0,30 \pm 0,66$ & $0,08 \pm 0,08$ & \\
\hline Médiane (Min-Max) & $0,10(0,03-0,87)$ & $0,04(0,01-0,29)$ & \\
\hline$r \mathbf{k}_{\text {trans }}$ & & & $0,035^{*}$ \\
\hline Moyenne \pm écart-type & $645 \pm 2130$ & $146,4 \pm 162,7$ & \\
\hline Médiane (Min-Max) & $156(11-804,8)$ & $95,4(7,6-807,9)$ & \\
\hline rIAUGC & & & $<0,001 *$ \\
\hline Moyenne \pm écart-type & $779 \pm 1616$ & $336 \pm 686$ & \\
\hline Médiane (Min-Max) & $404(130-1282)$ & 135 (49-3817) & \\
\hline $\mathbf{K}_{\mathrm{ep}}$ moyen & & & $0,359 *$ \\
\hline Moyenne \pm écart-type & $0,79 \pm 0,83$ & $0,60 \pm 0,56$ & \\
\hline Médiane (Min-Max) & $0,56(0,08-1,86)$ & $0,47(0,11-2,76)$ & \\
\hline
\end{tabular}

*test de Kruskal-Wallis

Tableau 5: Mesure de paramètres quantitatifs ( $k_{\text {trans }}$ moyen et $r k_{\text {trans, }} r$ IAUGC et $k_{\mathrm{ep}}$ moyen) par imagerie de perfusion $\mathrm{T} 1$ dans les deux groupes 

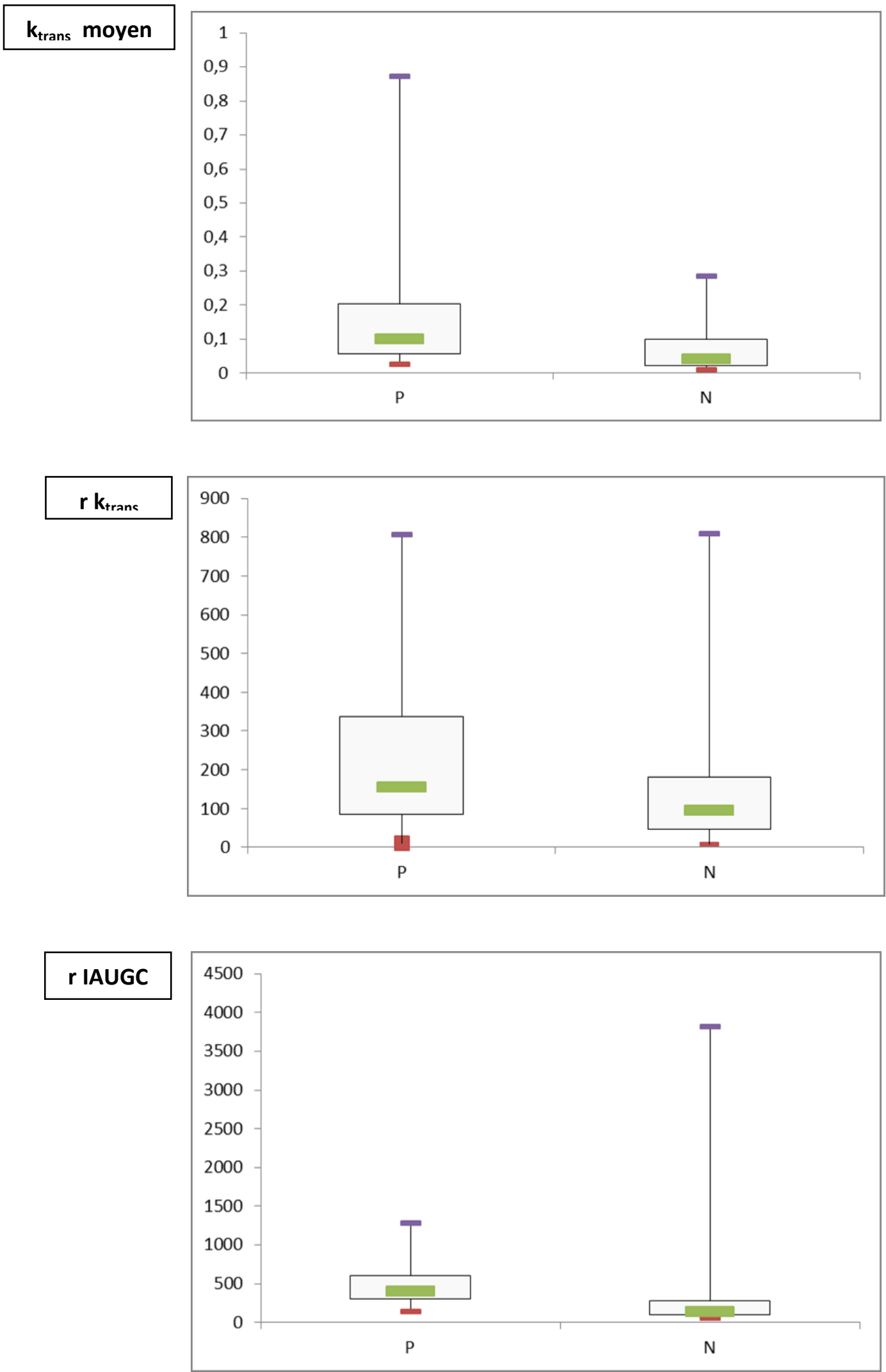

Figure 30 : Représentation des paramètres mesurés en imagerie de perfusion T1 sous forme de boîtes à moustache 
Les valeurs mesurées de $k_{\text {trans }}$ moyen, de $r k_{\text {trans }}$ et de $r$ IAUGC étaient significativement plus élevées dans le groupe $P$.

Les valeurs du coefficient $k_{e p}$ moyen n'étaient pas discriminantes entre les deux groupes.

II existait cependant une tendance avec des valeurs supérieures dans le groupe $\mathrm{P}$ sans différence statistiquement significative.

Par analogie à l'imagerie de perfusion $\mathrm{T} 2 *$, l'analyse discriminante linéaire a permis d'établir des seuils décisionnels.

Le meilleur classement obtenu était de $61 \%$ avec un seuil de $r$ IAUGC $=559,7$.

Pour les autres paramètres, les meilleurs classements obtenus étaient respectivement de $60 \%, 58,9 \%$ et $50,9 \%$ avec des seuils de $k_{\text {trans }}$ moyen $=0,2, r k_{\text {trans }}=395$ et de $k_{\text {ep }}$ moyen $=0,7$.

Le type de courbe de rehaussement ne différait pas significativement entre les deux groupes $(p=0,313)$. 


\subsubsection{Association des deux techniques d'imagerie de perfusion}

Une analyse utilisant la régression logistique binaire combinant le paramètre quantitatif $r$ VSC, calculé en imagerie de perfusion T2*, aux paramètres $r k_{\text {trans }}$ et $r$ IAUGC, mesurés en imagerie de perfusion $\mathrm{T1}$, a été réalisée.

Les paramètres $r$ VSC et $r k_{\text {trans }}$ étaient statistiquement significatifs ( $p=0,005$ et $p=0,045$ ) entre les deux groupes.

Les valeurs de r IAUGC ne différaient pas significativement en analyse multivariée ( $p=0,310)$.

4.2.2.4. Performances diagnostiques de l'imagerie de perfusion $\mathrm{T} 2 *$ et $\mathrm{T} 1$

Les paramètres, dont les valeurs mesurées en imagerie de perfusion $\mathrm{T} 2 *$ et $\mathrm{T} 1$, différaient statistiquement dans les deux groupes ont ensuite été analysés à l'aide de courbes ROC (Receiver Operating Characteristic), afin de déterminer lesquels étaient les plus discriminants par comparaison de leurs aires sous la courbe.

Les résultats étaient illustrés par la figure 31. 

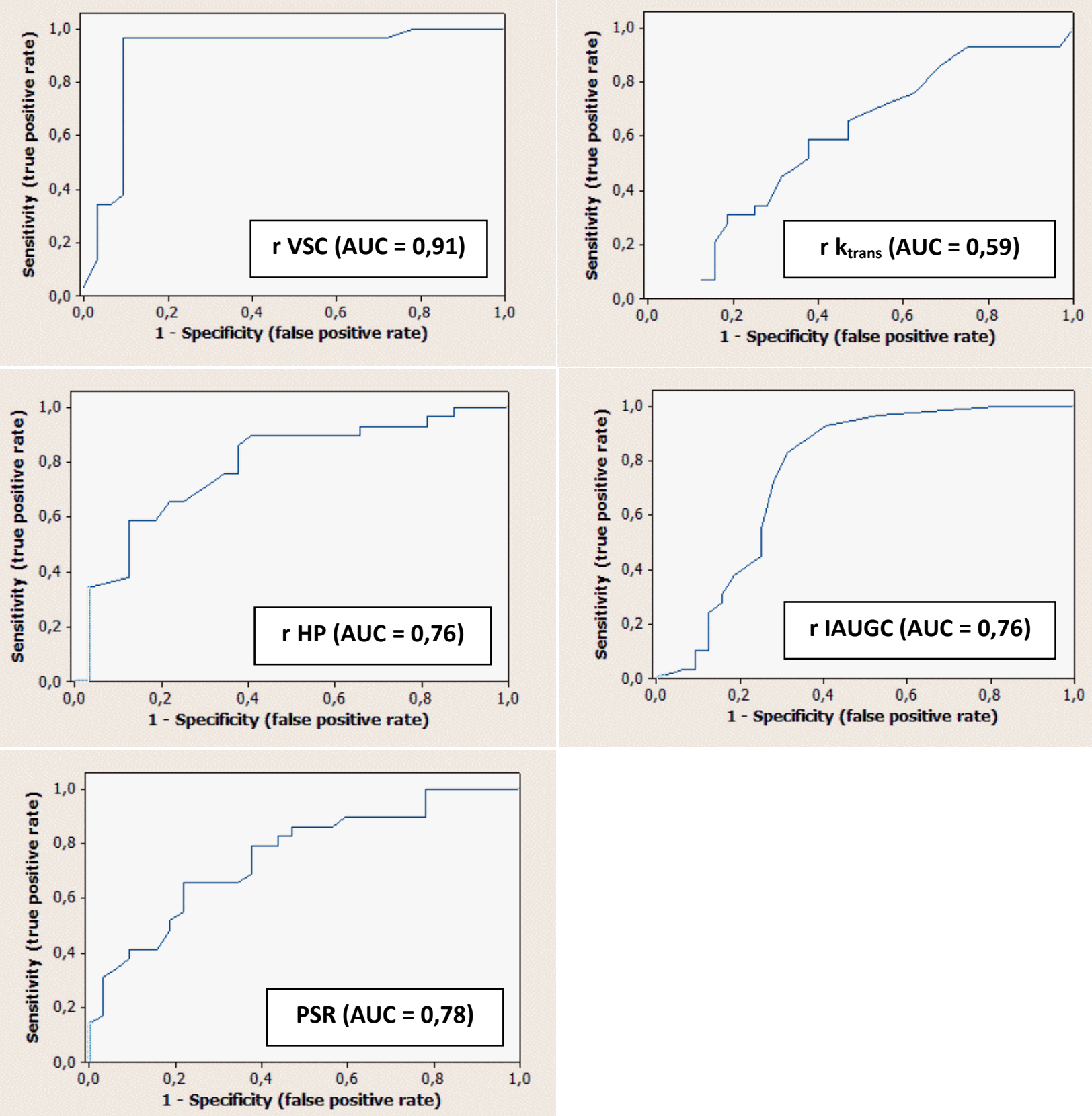

Figure 31 : Courbes ROC correspondant aux performances diagnostiques des paramètres en imagerie de perfusion

A gauche : paramètres $r$ VSC, $r$ HP et PSR mesurés en imagerie de perfusion T2*

A droite : paramètres $r k_{\text {trans }}$ et $r$ IAUGC mesurés en imagerie de perfusion T1 
4.2.2.5. Sur la séquence de diffusion

La moyenne des valeurs de $r$ ADC calculées dans le groupe $P$ était de $1,46 \pm 0,39 \mathrm{~mm}^{2} / \mathrm{s}$ $(\min : 0,90-\max : 2,57)$ versus $1,44 \pm 0,70 \mathrm{~mm}^{2} / \mathrm{s}(\min : 0,42-\max : 4,22)$.

II n'existait pas de différence significative entre les deux groupes $(p=0,359)$.

\subsubsection{Paramètres métaboliques}

Les ratios automatiquement calculés Cho/NAA et Cho/Cr étaient disponibles pour $56 \%$ des patients $(n=34)$. La moyenne des valeurs du ratio Cho/NAA dans le groupe $P$ était de 0,97 \pm $0,37(\min : 0,54-\max : 1,78)$ versus $1,12 \pm 0,83(\min : 0,58-\max : 3,91)$.

La moyenne des valeurs du ratio $\mathrm{Cho} / \mathrm{Cr}$ dans le groupe $\mathrm{P}$ était de $1,11 \pm 0,33$ ( $\min : 0,68$ $\max : 1,83)$ versus $1,13 \pm 0,31(\min : 0,78-\max : 1,76)$.

Ces deux paramètres quantitatifs ne différaient de façon significative entre les deux groupes (Cho/NAA ; $p=0,983$ et Cho/Cr ; $p=0,877$ ).

L'analyse qualitative du spectre montrait la présence de lipides et/ou lactates chez 56\% $(n=22)$ des patients du groupe $P$ et $44 \%(n=17)$ de ceux du groupe $N$, sans différence significative associée $(p=0,11)$.

Une élévation du pic de choline était observée chez $54 \%(n=13)$ des patients du groupe $P$ et $46 \%(n=11)$ de ceux du groupe N.

II n'existait pas de différence significative entre les deux groupes $(p=0,77)$.

Le spectre n'était pas interprétable dans $11 \%$ des cas $(n=6)$. 
4.2.4. Evaluation de la concordance des résultats de l'interprétation de l'IRM multimodale entre les lecteurs junior et senior

La concordance inter observateur entre les lecteurs junior et senior a été réalisée selon la méthode de Bland-Altman.

Elle compare les moyennes des mesures représentées en abscisse, à leurs différences représentées en ordonnée.

Chaque point représentait pour un patient, la différence entre les résultats obtenus par les deux lecteurs, par rapport à la moyenne des différences.

La moyenne des résultats (biais), l'écart-type et les limites de concordance supérieure et inférieure (moyenne \pm 1,96 écart-type) étaient calculées et illustrées par la figure 32 . 

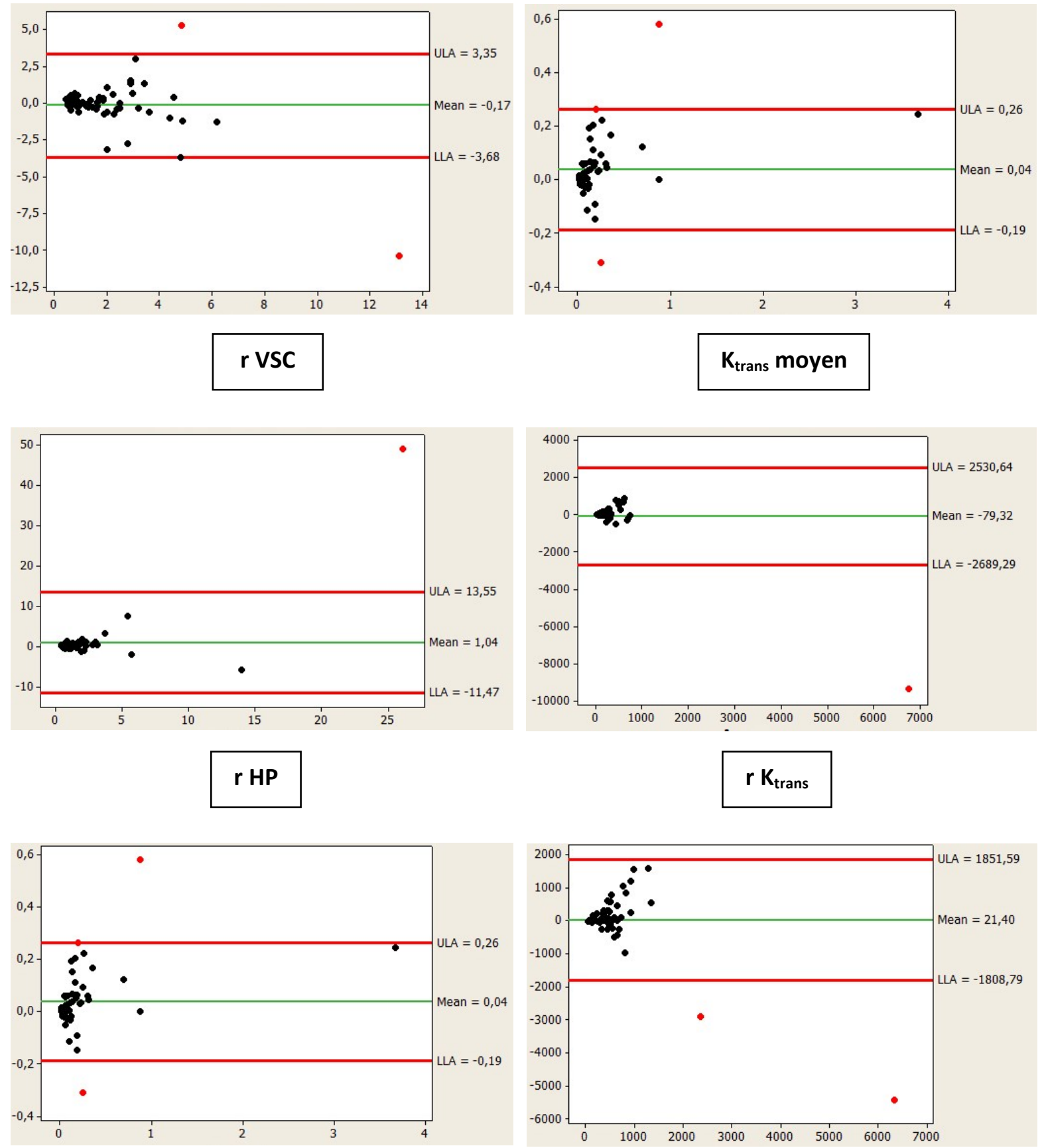

PSR

\section{r IAUGC}

Figure 32 : Diagrammes de Bland Altman représentant la reproductibilité des mesures de $r$ VSC, $r$ HP, PSR, $k_{\text {trans }}$ moyen, $r k_{\text {trans }}$ et $r$ IAUGC, entre les lecteurs junior et senior.

En vert : la ligne centrale continue représente le biais (ou moyenne des différences)

En rouge: les lignes discontinues supérieure et inférieure représentent les limites de concordance supérieure et inférieure 


\subsection{Evaluation des données relatives à la TEP-TDM à la ${ }^{18}$ F-FDOPA}

4.3.1. Performances diagnostiques de la TEP-TDM à la ${ }^{18}$ F-FDOPA par rapport au goldstandard

Les valeurs de sensibilité, spécificité, valeurs prédictives positive (VPP) et négative (VPN), les rapports de vraisemblance (RV) positifs et négatifs, ainsi que le coefficient kappa de Cohen concernant l'interprétation de la TEP-TDM à la ${ }^{18} \mathrm{~F}$-FDOPA par rapport au gold-standard considéré, étaient calculées et résumées dans le tableau 6.

\begin{tabular}{|c|c|}
\hline Paramètres & Estimation \\
\hline \multirow{2}{*}{ Sensibilité } & $96,60 \%$ \\
\hline \multirow{2}{*}{ Spécificité } & IC $95 \%=82,8 \%-99,4 \%$ \\
\hline \multirow{2}{*}{ VPP } & IC $95 \%=51,4 \%-82,0 \%$ \\
\hline \multirow{2}{*}{ VPN } & $73,70 \%$ \\
\hline \multirow{2}{*}{ RV+ } & IC $95 \%=58 \%-85 \%$ \\
\hline \multirow{2}{*}{ RV- } & $95,70 \%$ \\
\hline \multirow{2}{*}{ Coefficient Kappa } & IC $95 \%=79 \%-99,2 \%$ \\
\hline & IC $95 \%=2,53-3,8$ \\
\hline
\end{tabular}

Tableau 6 : Concordance et performances diagnostiques de la TEP-TDM à la ${ }^{18}$ F-FDOPA par rapport au gold-standard. 
4.3.2. Evaluation de la concordance des résultats de l'interprétation de l'IRM multimodale et de la TEP-TDM à la ${ }^{18}$ F-FDOPA

Le coefficient Kappa de Cohen, évaluant la concordance entre les deux modalités d'examen était de 0,65.

$84 \%$ des cas $(n=51)$ analysés en IRM multimodale et en TEP-TDM à la ${ }^{18}$ F-FDOPA étaient concordants.

$16 \%$ de cas $(n=10)$ analysés étaient discordants entre les deux modalités d'examen, dont quelques exemples sont illustrés ci-dessous. 

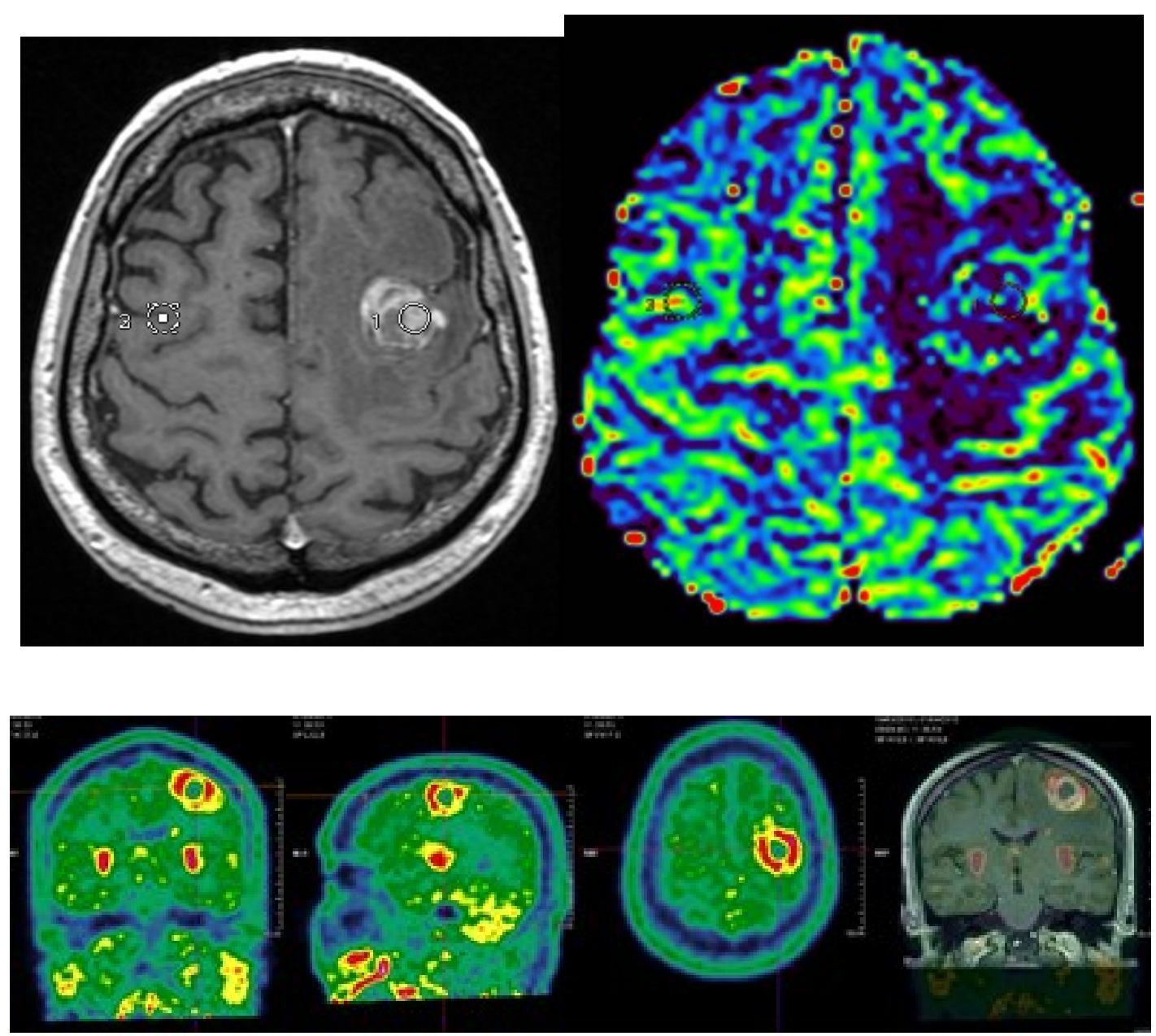

Figure 33 : Radionécrose histologiquement prouvée d'une métastase cérébrale secondaire à un primitif d'origine pulmonaire

En haut : IRM cérébrale montrant une hypo-perfusion de la lésion frontale gauche interprétée comme étant en faveur du diagnostic de radionécrose

En bas : Hyperfixation en périphérie de la lésion, d’intensité supérieure à celle des striata, ayant fait posé le diagnostic de récidive en TEP-TDM à la ${ }^{18}$ F-FDOPA 

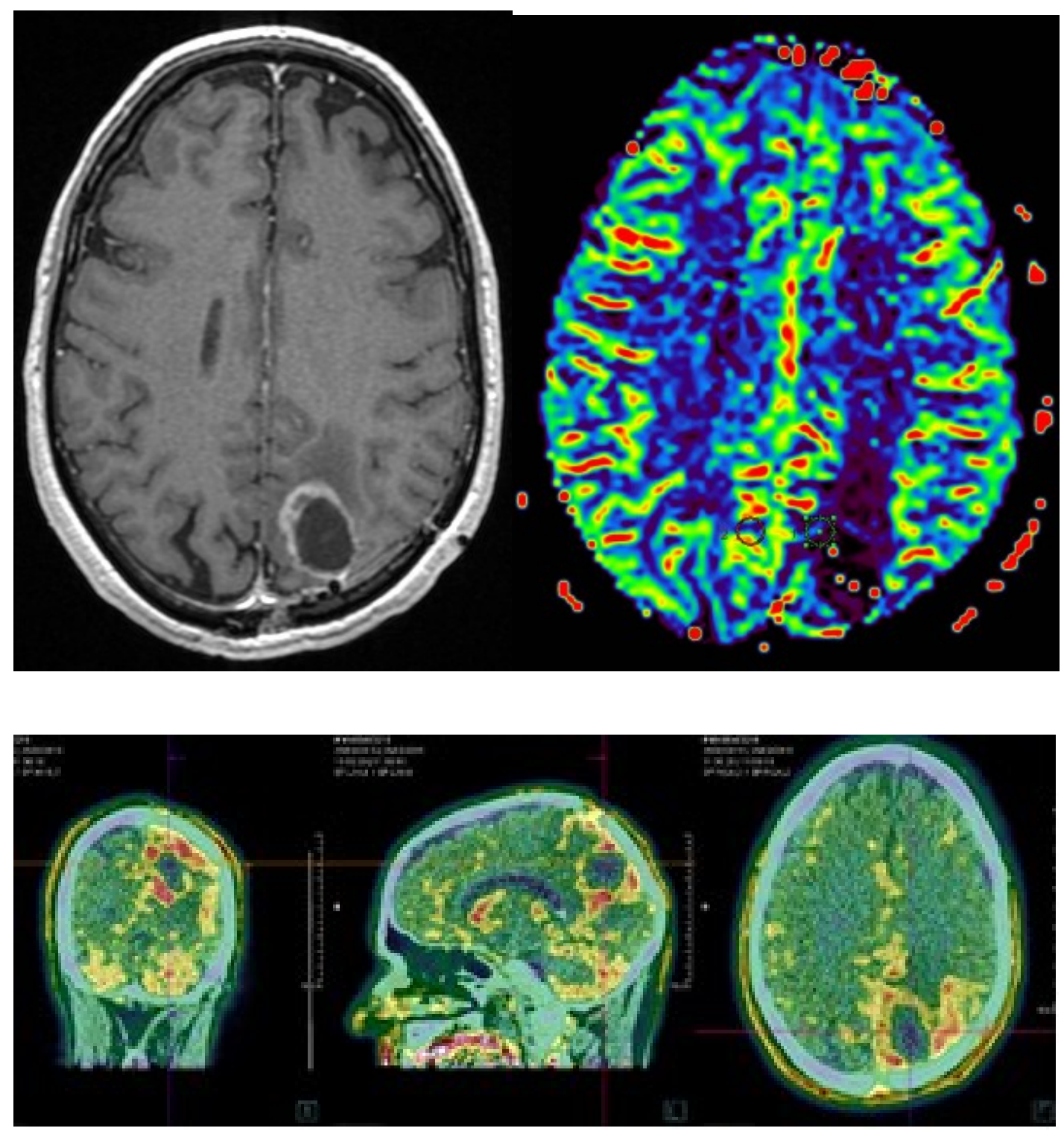

Figure 34 : Autre cas de radionécrose histologiquement prouvée d'une métastase cérébrale secondaire à un primitif d'origine pulmonaire

En haut : IRM cérébrale montrant une hypo-perfusion de la lésion occipitale gauche interprétée comme étant en faveur du diagnostic de radionécrose

En bas : Hyperfixation en périphérie de la lésion, d’intensité supérieure à celle des striata, ayant fait posé le diagnostic de récidive en TEP-TDM à la ${ }^{18}$ F-FDOPA 

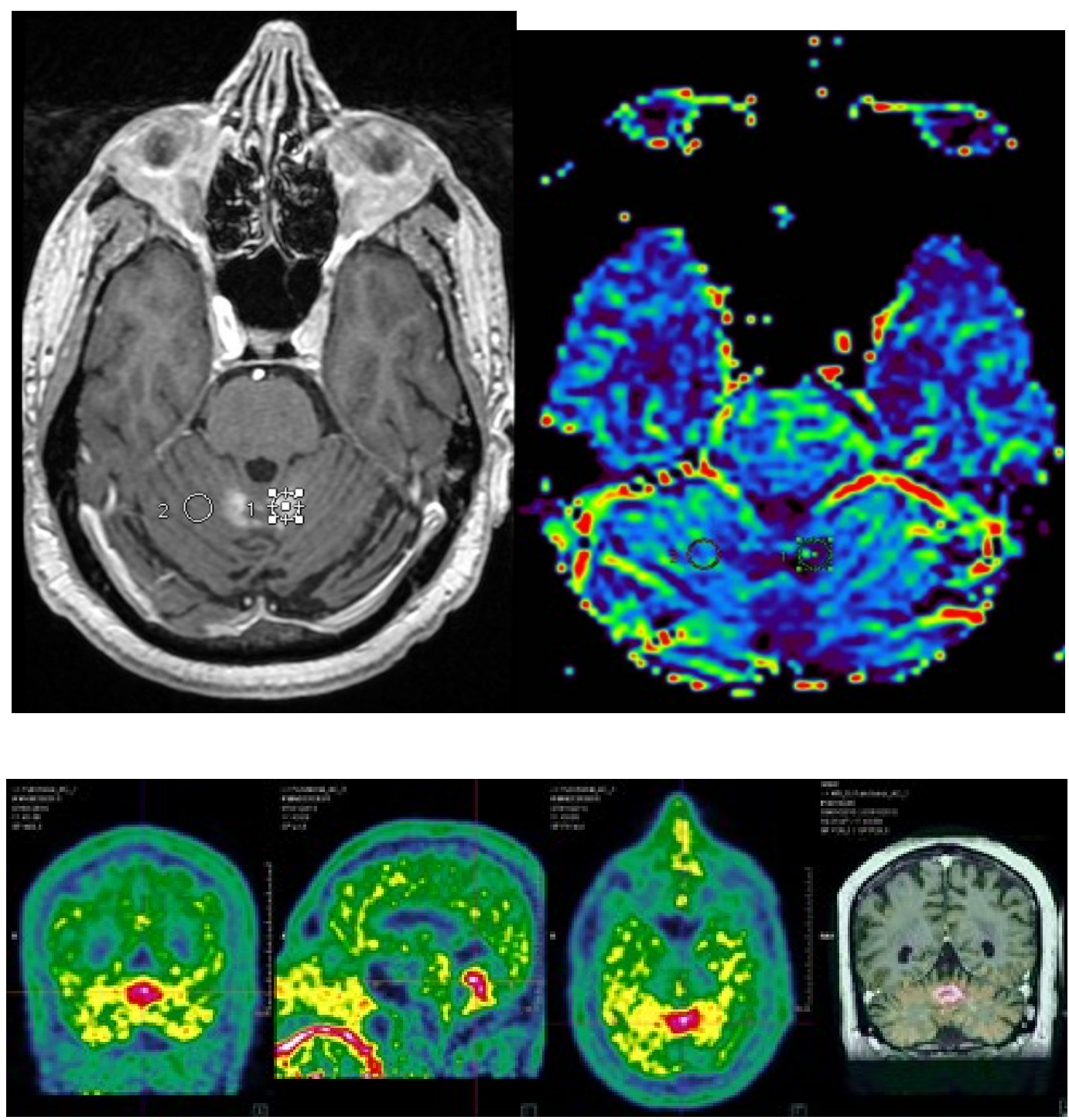

Figure 35 : Métastase cérébrale d'un primitif d'origine pulmonaire

En haut : Lésion vermienne présentant une prise de contraste floue avec absence d'hyper-perfusion évocatrice de radionécrose

En bas : Hyperfixation intense (d’intensité supérieure à celle des striata) de la lésion vermienne en TEP-TDM à la ${ }^{18}$ F-FDOPA 


\section{5 - DISCUSSION}

L'IRM multimodale est la modalité d'imagerie de référence pour le suivi des tumeurs cérébrales irradiées, combinant la réalisation de séquences morphologiques, fonctionnelles et métaboliques.

L'interprétation de l'ensemble des séquences du protocole d'IRM multimodale réalisé dans notre centre a montré une excellente concordance avec les données du gold-standard.

Notre étude se distingue par son caractère prospectif.

Nous avons évalué une combinaison d'outils morphologiques, fonctionnels et métaboliques, plus ou moins informatifs et discriminants pour distinguer radionécrose et récidive tumorale.

Dans notre étude, la présence d’un rehaussement lésionnel est significativement corrélée aux cas de progression tumorale.

Cependant, de manière comparable aux résultats précédemment publiés dans la littérature $[64,65]$, le type de pattern de rehaussement n'a pas été retenu comme discriminant pour distinguer les cas de radionécrose et ceux de récidive tumorale.

Peu de publications $[94,133]$ ont étudié la valeur ajoutée des techniques d'imagerie de perfusion $\mathrm{T} 1$ et $\mathrm{T} 2 *$ dans le suivi des tumeurs cérébrales irradiées. 
L'imagerie de perfusion T2* est utilisée en routine, en imagerie neuro-oncologique, donnant accès à des informations concernant la microcirculation capillaire, essentiellement via la mesure du volume sanguin cérébral.

Les phénomènes de néo-angiogenèse impliqués en cas de tumeur expliquent l'augmentation de $r$ VSC observée en cas de récidive.

Nous rapportons des valeurs de $r$ VSC significativement augmentées en cas de progression tumorale et diminuées en cas de radionécrose.

Les valeurs médianes mesurées dans notre série étaient respectivement de 2,26 et 0,72 dans le groupe $P$ et $N$, avec un seuil décisionnel calculé de $r$ VSC supérieur à 2,2, en cas de progression tumorale.

Ces données sont en accord avec les valeurs publiées par Sugahara et al [132] qui avait proposé un seuil de r VSC de 2,6 en cas de récurrence tumorale.

D'autres équipes $[76,87,78,134]$ ont plus récemment avancées des valeurs seuils de $r$ VSC comprises entre 1,7 et 2,38 en cas de récidive tumorale.

Il a été rapporté à de nombreuses reprises que la mesure du $r$ VSC est sous l'influence de plusieurs facteurs, pouvant expliquer les différences de valeurs seuils obtenues selon les séries.

Des facteurs individuels avec des variations inter et intra-observateurs ont été décrites dans la littérature, pouvant atteindre jusqu'à 30\% [86] chez un même patient.

Ces différences sont accentuées en cas de tumeur hétérogène et angiogénique. 
La figure 36 illustre un cas de notre population d'étude, suivi pour métastase cérébrale secondaire à un primitif d'origine mammaire, présentant une forme hyper-vascularisée de récidive tumorale, avec des valeurs de r VSC anormalement élevées.

Habituellement, les valeurs oscillent entre 3 et 4 [83].

Le placement erroné et subjectif de la ROI peut également entraîner une surestimation des valeurs de r VSC. Prenons l'exemple des glioblastomes, tumeurs associant des zones hypervascularisées appelées "hot spots » et des zones normalement perfusées voire hypoperfusées en rapport avec la présence de nécrose au sein de la tumeur [92].

Selon la zone d'intérêt choisie, la mesure de r VSC sera variable.

Des facteurs liés à la tumeur sont aussi impliqués. La proximité de la lésion avec une structure vasculaire peut simuler à tort une hyper-perfusion tumorale.

Des remaniements hémorragiques intra-tumoraux peuvent causer des effets de susceptibilité magnétique T2* [73], et modifier les valeurs de $r$ VSC.

Des altérations de la barrière hémato-encéphalique peuvent en outre générer des modifications de la perméabilité vasculaire. Elles s'accompagnent d'extravasation de produit de contraste, depuis le secteur vasculaire vers le secteur extravasculaire, responsable de variations de $\mathrm{T} 1$ et $\mathrm{T} 2 *$. Ces dernières induisent des modifications de la courbe de premier passage et par conséquent du r VSC. 


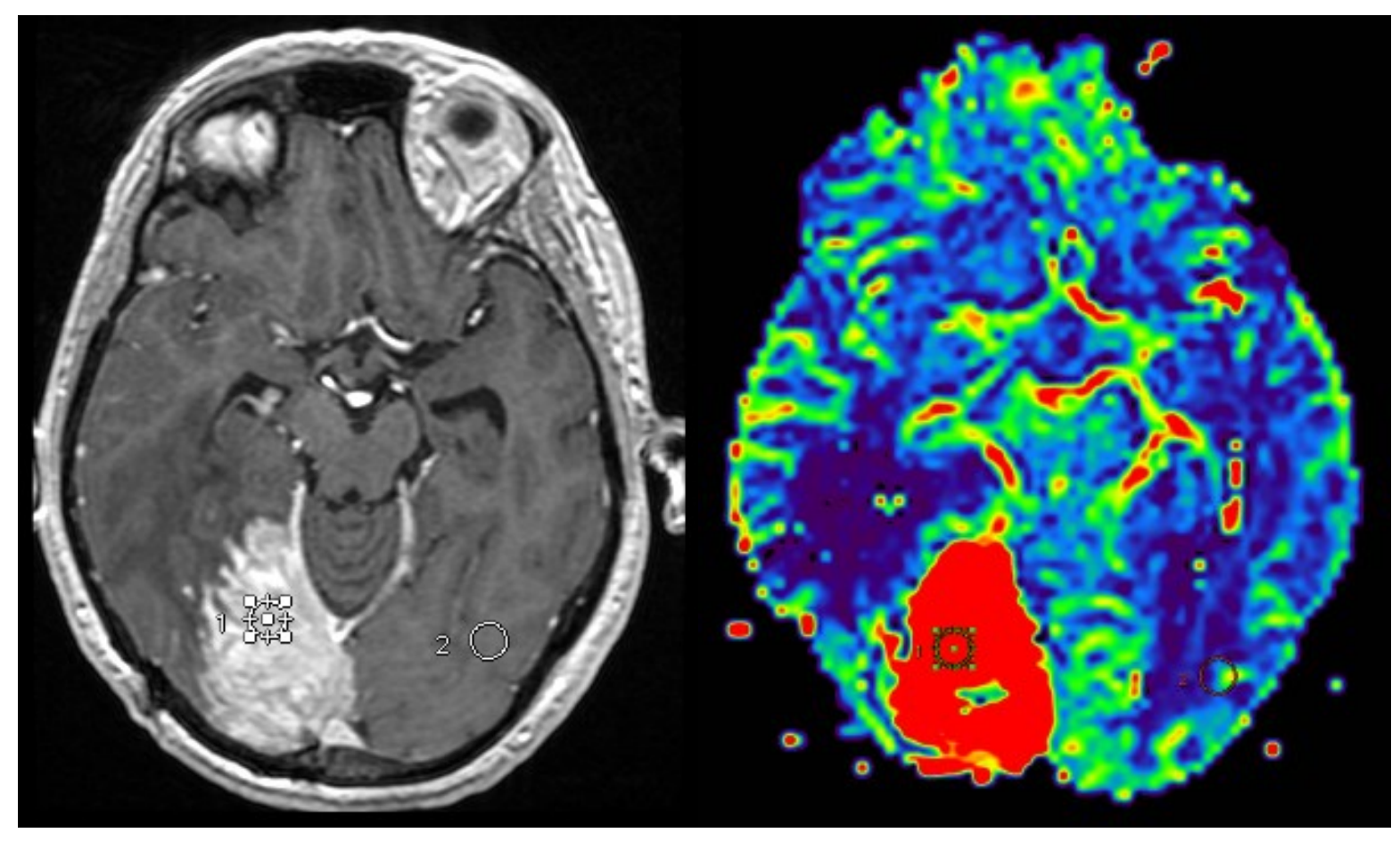

Figure 36 : Forme hyper-vascularisée de métastase cérébrale observée dans notre série

Un autre facteur influençant les valeurs de $r$ VSC concerne la prise d'un traitement antiangiogénique par Bevacizumab [135]. En inhibant la néo-angiogenèse tumorale et en restaurant la barrière hémato-encéphalique, cette molécule induit une diminution de la prise de contraste et du $r$ VSC, qui peut néanmoins être concomitante d'une progression tumorale.

L'intérêt des séquences pondérées en T2 [142] et T2 Flair [143] a été rapporté pour individualiser les zones non rehaussées, infiltrantes, évocatrices de progression tumorale. Compte-tenu de ce problème, la réponse radiologique au traitement des tumeurs gliales doit désormais être évaluée selon les critères RANO (Response Assesment in Neuro Oncology), présentés en annexe. 
En plus de l'imagerie pondérée en T1 après injection de gadolinium, ils prennent en compte l'imagerie T2 FLAIR pour ne pas méconnaître les cas de « pseudo-réponse ».

Il s'agit des cas au cours desquels il existe une disparition de la prise de contraste sous traitement anti-angiogénique, alors même que la tumeur progresse sous forme d'une extension de l'infiltration en imagerie pondérée en T2 FLAIR.

Dans notre série, bien que les séquences pondérées en T2 n'aient pas été intégré aux données relevées, ce qui peut être une source potentielle de biais, l'administration de Bevacizumab a été prise en compte et n'a pas influencé de façon significative les valeurs de $r$ VSC rapportées.

L'ensemble de ces écueils [136] ont conduit au développement d'autres critères d'évaluation en imagerie de perfusion T2* : le $r$ HP et le PSR.

Les valeurs que nous rapportons sont significativement concordantes avec celles décrites par Barajas $[87,88]$.

Les valeurs mesurées de $r$ HP sont plus élevées chez les patients présentant une récidive tumorale, corrélées aux résultats du r VSC.

Inversement, le PSR était significativement plus bas en cas de récidive tumorale.

Dans notre série, le $r$ VSC possède les meilleures performances diagnostiques $(A \cup C=0,91)$, suivi du PSR $(A \cup C=0,78)$ et du $r$ HP $(A \cup C=0,76)$ parmi les outils disponibles en imagerie de perfusion $\mathrm{T}^{*}$. 
Nous nous sommes intéressés au potentiel de l'imagerie de perfusion $\mathrm{T} 1$, technique plus précise permettant une modélisation de la fuite de l'agent de contraste, dans le suivi des tumeurs cérébrales irradiées.

Nous rapportons des valeurs de $k_{\text {trans }}$ moyen, $r k_{\text {trans }}$ et $r$ IAUGC, paramètres mesurés en imagerie de perfusion T1, significativement augmentés en cas de récidive tumorale.

Au contraire, le coefficient $k_{e p}$, malgré une tendance à des valeurs supérieures en cas de progression tumorale, n'est pas un paramètre retenu comme discriminant, comme cela a déjà été rapporté $[94,133,137]$.

La combinaison des deux techniques de perfusion réalisée dans notre protocole est pertinente pour distinguer les cas de radionécrose et de récidive tumorale.

L'association de valeurs augmentées de $r$ VSC (mesuré en imagerie de perfusion T2*) et de $r k_{\text {trans }}$ (mesuré en imagerie de perfusion T1) est corrélée de façon significative aux cas de progression tumorale, en accord avec les résultats rapportés par Shin et al [94].

La place de l'imagerie de diffusion a fait l'objet de nombreuses publications, avec des résultats contrastés selon les études [74, 75, 76, 138], significatifs ou non.

Ces variations s'expliquent, selon les séries publiées, par des effectifs réduits, un design et des conditions de réalisation différents entre les études.

Un autre problème majeur concerne l'existence de lésions mixtes, c'est-à-dire associant des lésions de récidive tumorale à des lésions post-radiques. 
Les valeurs de $r$ ADC relevées dans notre étude, bien que plus basses en cas de progression tumorale, ne différaient pas significativement entre les deux groupes.

Le constat global est celui de valeurs de $r$ ADC plus élevées en cas de radionécrose, du fait d'une augmentation des mouvements des molécules d'eau.

Inversement, en cas de progression tumorale, il existe une augmentation de la cellularité, responsable d'une restriction des mouvements des molécules d'eau, et donc de valeurs d'ADC diminuées.

Les données récentes rapportées par la méta-analyse de Zhang et al [139] concordent avec nos résultats, considérant que l'imagerie de diffusion a des performances diagnostiques modérées pour distinguer les cas de radionécrose et de récidive tumorale, et doit être associée aux autres séquences d'IRM multimodale.

Nous avons également étudié l'intérêt des données spectroscopiques pour différencier les deux entités.

Le concept d'un spectre distinct en cas de radionécrose ou de progression tumorale n'est qu'utopique, car comme nous l'avons déjà précisé plus haut, une association lésionnelle est souvent rapportée.

Dans notre étude, les ratios calculés en spectroscopie $\mathrm{Cho} / \mathrm{Cr}$ et Cho/NAA ne diffèrent pas significativement selon les groupes.

Les données de la littérature relatives aux paramètres quantitatifs mesurés par la séquence de spectroscopie sont discordantes parmi les séries publiées $[76,140]$. 
Les différences peuvent être expliquées conjointement par la variabilité des effectifs, le polymorphisme lésionnel et le type de spectroscopie réalisé (mono ou multi-voxel).

Dans notre série, un facteur limitant surajouté est l'absence de calcul automatique des ratios pour $44 \%$ des patients.

II faut également prendre en considération d'autres sources d'erreurs tels qu'un positionnement erroné ou inadapté du voxel au sein de la lésion étudiée ou encore la proximité de certaines lésions avec des structures osseuses, risquant de fausser les résultats attendus.

L'analyse qualitative spectrale n'a pas non plus montré de différence entre les deux groupes concernant la présence de lipides et / ou lactates et l'élévation du pic de choline.

Les lipides traduisent la survenue de phénomènes de nécrose cellulaire. Leur présence est souvent individualisée en cas de tumeur [141].

De même, il est fréquent d'observer un pic de lipides prédominant effaçant tous les autres pics, en cas de radionécrose.

L'augmentation de choline, marqueur du métabolisme membranaire, traduit une cellularité élevée ou la présence de phénomènes inflammatoires, lesquels sont respectivement impliqués en cas de progression tumorale et de radionécrose.

Nos résultats s'accordent avec les conclusions d'une méta-analyse récente [140] qui considère la spectroscopie comme une technique dont les performances diagnostiques sont limitées pour différentier radionécrose et récidive tumorale. 
Son utilisation nécessiterait, comme pour l'imagerie de diffusion, l'intégration aux autres séquences réalisées lors de l'interprétation de l'IRM multimodale.

Nous avons aussi apprécié la concordance des résultats rapportés, dont l'analyse statistique a été réalisée chez le lecteur junior, avec ceux du lecteur senior, spécialisé en neuroradiologie.

Il existe une reproductibilité satisfaisante des mesures, avec une variabilité intra observateur faible.

Les valeurs situées en dehors des limites de concordance sur les diagrammes de BlandAltman suggèrent des valeurs aberrantes, évoquant soit des erreurs de saisie ou de mesure, soit des cas complexes comme celui de la forme hyper-vascularisée de tumeur gliale de haut grade évoquée précédemment, expliquant les variations entre les deux lecteurs.

Parallèlement à l'évaluation des meilleurs outils en IRM multimodale, notre étude se distingue par l'analyse de la concordance de ces résultats avec les données d'un examen de médecine nucléaire de référence pour le suivi des tumeurs cérébrales, la TEP-TDM à la ${ }^{18} \mathrm{~F}$ FDOPA.

A notre connaissance, aucune étude publiée n'a comparé les deux modalités d'examen, en incluant parmi les données IRM, I'utilisation des deux techniques de perfusion T1 et T2*. 
Si l'intérêt de la TEP-TDM à la ${ }^{18}$ F-FDOPA est bien connu en neuro-oncologie [144] compte tenu de sa faible fixation physiologique dans le parenchyme cérébral sain, peu d'études à ce jour ont comparé les deux modalités.

L'une d'elles a comparé I'IRM cérébrale injectée avec la TEP-TDM à la ${ }^{18}$ F-FDOPA montrant une efficacité diagnostique comparable entre les deux modalités, avec cependant une meilleure spécificité pour la TEP-TDM à la ${ }^{18}$ F-FDOPA [131].

Une autre étude s'est intéressée aux performances de l'IRM de perfusion T2* et notamment de la mesure $r$ VSC comparativement à la TEP-TDM à la ${ }^{18} \mathrm{~F}$-FDOPA, pour différentier les lésions de radionécrose et de récidive dans le cas spécifique des métastases cérébrales irradiées. D'après les résultats rapportés, cet examen serait un outil très efficace et présenterait une meilleure spécificité que I'IRM de perfusion dans ce cas précis [130], en utilisant des paramètres semi-quantitatifs.

Nous avons suivi la méthode d'interprétation réalisée dans notre centre, basée principalement sur une évaluation qualitative de l'examen reposant sur l'échelle visuelle.

Ce paramètre a d'ailleurs montré la meilleure sensibilité et spécificité pour la détection d'une progression tumorale, dans l'étude de Lizarraga [128].

Dans notre série, l'évaluation en IRM multimodale a intégré l'ensemble des séquences et paramètres. 
La concordance observée entre les deux modalités est bonne.

L'analyse parallèle de la concordance de la TEP-TDM à la ${ }^{18}$ F-FDOPA avec le gold-standard montre une excellente sensibilité et une spécificité modérée de cet examen dans notre étude.

Nous nous sommes intéressés avec une attention particulière aux cas discordants, recensés lors de l'analyse des résultats rapportés de l'examen TEP-TDM à la ${ }^{18} \mathrm{~F}$-FDOPA par rapport à l'interprétation de l'IRM multimodale.

L’analyse effectuée a permis de mettre en évidence essentiellement deux situations.

La première concerne les métastases cérébrales.

Plusieurs cas avérés de radionécrose (dont certains sont illustrés par les figures 33 et 34) se sont révélés être des cas « faux positifs » de la TEP-TDM à la ${ }^{18} \mathrm{~F}$-FDOPA, dans notre série.

II existait une hyperfixation intense de ces lésions, qui contrastait avec l'absence d' hyperperfusion ou de signe évocateur de récidive tumorale observé en IRM.

Il est maintenant connu que l'intensité de fixation à la TEP-TDM à la ${ }^{18} \mathrm{~F}$-FDOPA est corrélée à l'expression de LAT $1[145,146]$.

Les données récentes rapportées lors d'une thèse [147] ayant étudié l'expression de LAT 1 dans les métastases cérébrales ont mis en évidence une surexpression de LAT 1 dans le tissu cérébral irradié, dont les mécanismes physiopathologiques sont encore mal connus. 
Ce constat pourrait expliquer certains cas faux positifs de notre série, en rapport avec une hyperfixation intense en TEP-TDM à la ${ }^{18} \mathrm{~F}-\mathrm{FDOPA}$, pouvant être expliquée par une surexpression de LAT 1 dans les cas de radionécrose.

Une autre étude [148], réalisée chez 21 patients ayant bénéficié d'une imagerie par TEPTDM à la ${ }^{18}$ F-FDOPA pour différentier récidive et radionécrose tumorale, a appuyé ces données montrant 2 cas "faux positifs ". Ces derniers présentaient une surexpression de LAT 1 dans le tissu cérébral nécrotique irradié.

La seconde situation fait référence à deux cas de notre série, dont un est représenté par la figure 35 .

Il s'agit de lésions de la fosse postérieure, pour lesquelles il existait une discordance entre les deux modalités.

Alors que la TEP-TDM à la ${ }^{18} \mathrm{~F}$-FDOPA montre une hyperfixation intense de ces lésions, suspecte de récidive, l'IRM est évocatrice de radionécrose.

Pour ces deux cas, le suivi a été biaisé pour l'un par le décès du patient et pour l'autre, par l'absence d'imagerie de contrôle.

Nous n'avons pas trouvé de publications concernant la spécificité de la TEP-TDM à la ${ }^{18} \mathrm{~F}$ FDOPA pour l'étude des lésions de la fosse postérieure.

Il serait intéressant de poursuivre le suivi de ces lésions, à plus long terme, pour confirmer ces données ou au mieux d'obtenir une preuve anatomo-pathologique. 
II serait attrayant d'analyser plus précisément la concordance entre ces deux modalités. L'évaluation de la concordance, partielle ou totale, entre la fixation en TEP-TDM à la ${ }^{18} \mathrm{~F}$ FDOPA et les cartographies paramétriques obtenues en IRM multimodale, par fusion d'images, pourrait apporter des informations supplémentaires.

II convient également de signaler un cas de notre série, illustré par la figure 37, pour lequel les deux modalités d'examen ont été prises en défaut. 


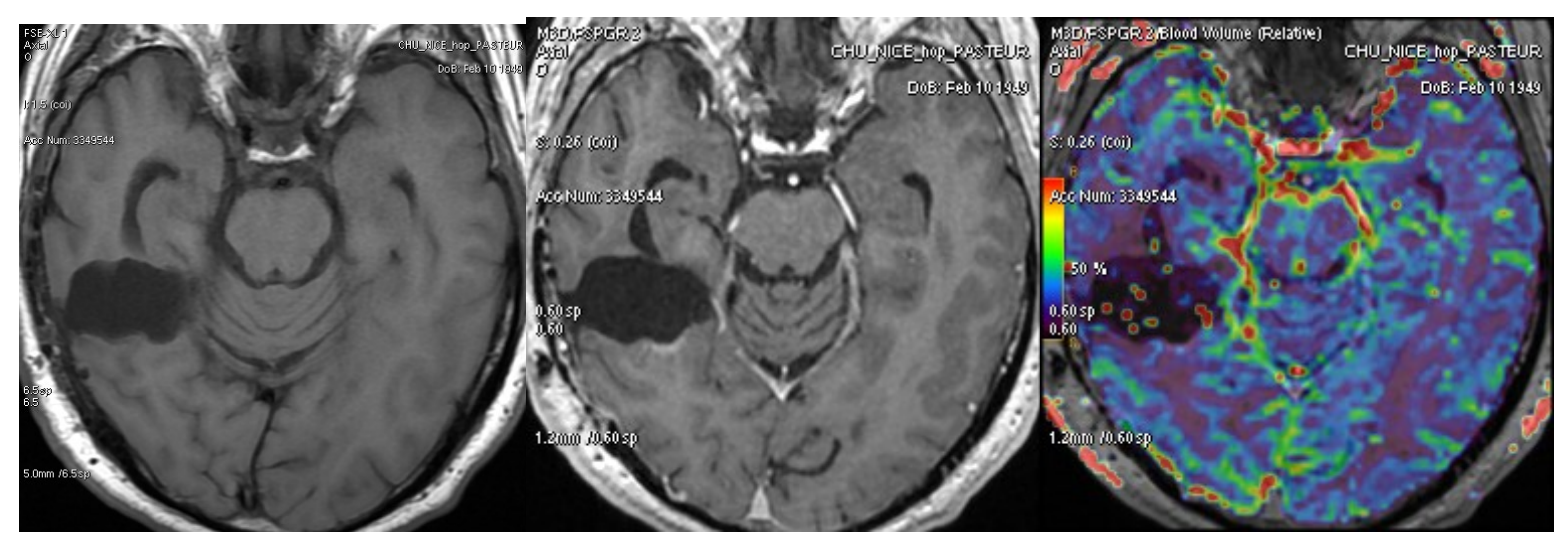

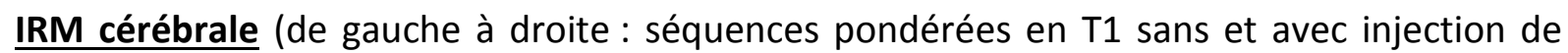
gadolinium, et cartographie de VSC) montrant une minime prise de contraste périphérique, sans hyper-perfusion associée.

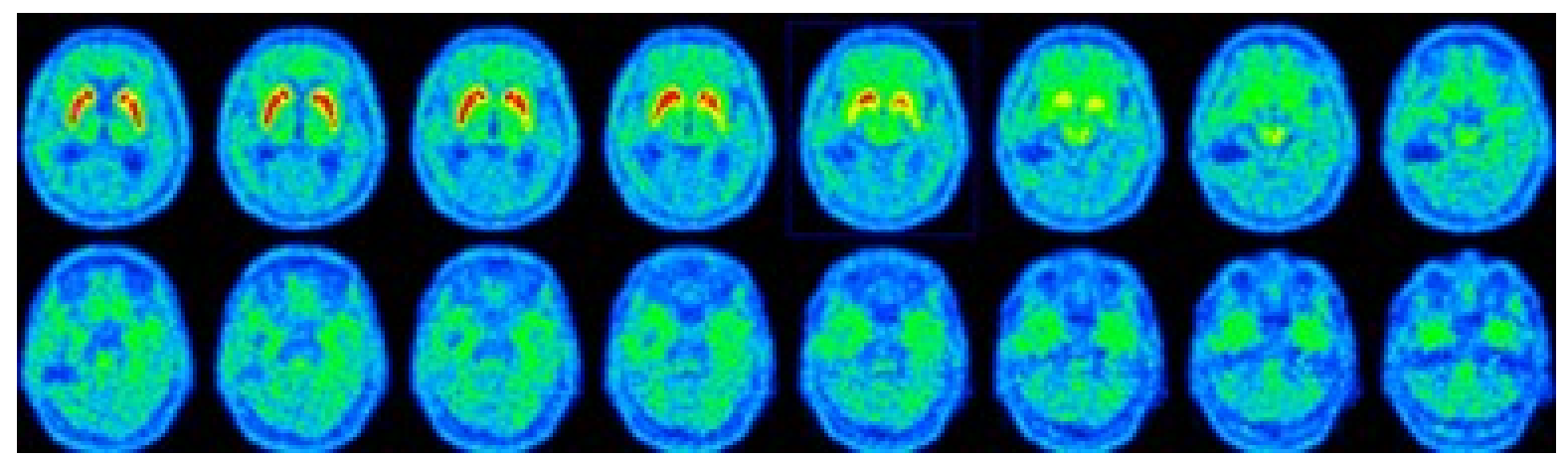

$\underline{\text { TEP-TDM à la }{ }^{18} \text { F-FDOPA montrant une absence de fixation du radio-pharmaceutique. }}$

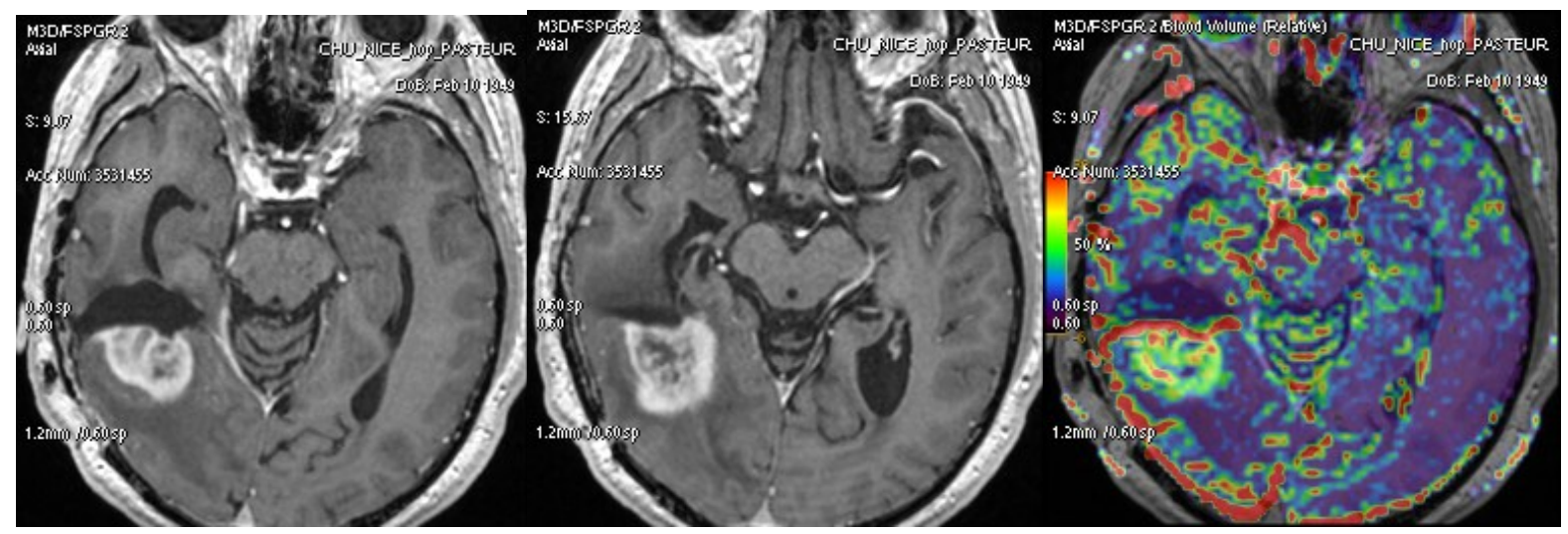

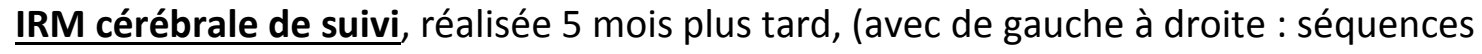
pondérées en T1 avec injection de gadolinium et cartographie de VSC) montrant la majoration de la prise de contraste, avec hyper-perfusion lésionnelle.

Figure 37 : Absence de diagnostic précoce des deux modalités d'examen. 
Le suivi clinico-radiologique de ce patient a permis de mettre en évidence une progression tumorale, qui n'avait pas été détectée précocement en IRM multimodale et en TEP-TDM à la ${ }^{18}$ F-FDOPA.

Ce travail présente quelques limites qu'il est nécessaire de détailler.

Le nombre de patients est faible, limitant les analyses statistiques et la puissance de l'étude, même si le collectif analysé est numériquement supérieur à la plupart des études publiées.

Une autre limite concerne la dichotomisation entre radionécrose et récidive tumorale réalisée dans notre étude.

La catégorie de patients présentant une radionécrose incluait de potentiels cas de pseudoprogression, parmi les tumeurs gliales.

Néanmoins, l'interprétation de l'IRM multimodale et plus précisément des séquences de diffusion et de perfusion [138, 149] en cas de pseudo-progression montrent des résultats similaires aux cas de radionécrose observées dans notre série. En effet, des valeurs plus faibles de $r$ VSC et de $r$ HP, et plus élevées de PSR ont été rapportées en cas de pseudoprogression par rapport aux cas de progression tumorale [149].

De même, les patients présentant une stabilité lésionnelle ont été intégrés aux cas de radionécrose dans notre série.

Une limite supplémentaire repose sur le polymorphisme lésionnel car nous avons inclus à la fois des tumeurs gliales et des métastases cérébrales, bien que les proportions des deux types de tumeurs étaient quasiment identiques dans notre population d'étude. 
Parmi les tumeurs gliales, les tumeurs de bas grade représente aussi un écueil, bien qu'en nombre limité dans notre étude, compte tenu de l'absence de rehaussement de celles-ci par définition. 


\section{6 - CONCLUSION}

L'IRM multimodale est l'imagerie de référence pour le suivi des tumeurs cérébrales irradiées.

L'analyse d'outils morphologiques, fonctionnels et métaboliques pour distinguer radionécrose et récidive tumorale a permis de mettre en évidence l'intérêt des deux techniques d'imagerie de perfusion $\mathrm{T} 1$ et $\mathrm{T} 2^{*}$ dans cette indication.

Notre étude valide l'utilisation du r VSC, paramètre dérivé de l'imagerie de perfusion T2* et $r K_{\text {trans, }}$ issu des données d'imagerie de perfusion T1 pour différentier radionécrose et progression tumorale.

Les autres outils morphologiques, métaboliques ou encore l'apport de l'imagerie de diffusion ne sont pas discriminants pour répondre à cette problématique.

La TEP-TDM à la ${ }^{18} \mathrm{~F}$-FDOPA présente une bonne concordance avec les données de l'IRM multimodale, avec néanmoins une spécificité modérée liée entre autres à une surexpression de LAT 1 dans le tissu cérébral irradié, source de faux positifs. 


\section{7 - BIBLIOGRAPHIE}

1. Bailey $\mathrm{P}$, Cushing H.A classification of the tumors of the glioma group on a histogenetic basis with a correlated study of prognosis. Philadelphia: JB Lippincott; 1926.

2. Kernohan JW, Sayre GP.Tumors of the central nervous system. Atlas of tumor pathology (fasc 35), Washington: Armed Forces Institute of Pathology; 1952.

3. Zülch KJ. Types histologiques du système nerveux central. Genève: OMS; 1979.

4. Kleihues P, Burger PC, Scheithauer BW.Histological typing of tumours of the central nervous system.World Health Organization international histological classification of tumours. Heildelberg: Springer; 1993.

5. Kleihues P, Cavenee WK. World Health Organization Classification of Tumours. Pathology and genetics of tumours of the nervous system. Lyon: IARC Press; 2000.

6. LOUIS DN et al.The 2007 WHO classification of tumours of the central nervous system. Acta Neuropathol 2007: 11; 97-109.

7. ENCR, European Network of Cancer Registries. Recommendations for coding Tumours of the Brain and Central Nervous System, 1998.

8. Ohgaki H, Kleihues P. Epidemiology and etiology of gliomas. Acta Neuropathol. 2005 Jan; 109(1):93-108]

9. CBTRUS Statistical Report: Primary Brain and Central Nervous SystemTumors Diagnosed in the United States in 2004-2008.Central Brain Tumors Registry of the United States.2012. 
10. Ostrom QT1, Gittleman H, Stetson L, Virk SM, Barnholtz-Sloan JS. Epidemiology of gliomas. Cancer Treat Res. 2015; 163:1-14.

11. Burkhard C., Di Patre PL, Schüler D, Yasargil MG, Yonekawa Y, Lutolf UM, Kleihues P, Ohgaki H. A population-based study of the incidence and survival rates in patients with astrocytic pilocytoma. J Neurosurg. 2003 Jun; 98(6):1170-4.

12. Morgan LL. The Epidemiology of glioma in adults: a " state of the science " review. Neuro Oncol 2015 Apr; 17(4):623-4.

13. Sathornsumetee S, Rich JN, Reardon DA.Diagnosis and treatment of high-grade astrocytoma. Neurol Clin 2007; 25:1111-39.

14. Daumas-Dupont $C$, Tucker $M L$, Kolles $H$, Cervera $P$, Beuvon $F$, Varlet $P$, et al. Oligodendrogliomas, II: a new grading system based on morphological and imaging criteria. J Neurooncol 1997; 34: 61-78.

15. Inbaih A., Duran-Pena A, Bonnet C, Ducray F. Input of molecular analysis in medical management of primary brain tumor patients. Rev Neurol(Paris). 2015 Jun-Jul; 171(6-7):45765.

16. Delmaire C, Savatovsky J, Boulanger T, Dhermain F, et al.Brain metastases imaging. Cancer Radiother. 2015 Feb; 19(1):16-9.

17. Taillebert S, Le Rhun E Epidemiology of brain metastases. Cancer Radiother. 2015 Feb; 19(1):3-9. 
18. Grand S, Pasteris C, Attye A, Le Bas J-F, Krainik A. Les différents visages des métastases du système nerveux central. Journal de Radiologie Diagnostique et Interventionnelle. 2014; 95:908-22.

19. Delarive J., de Tribolet N. Cerebral metastases. A study of a surgical series of 81 cases Neurochirurgie 1992; 38: 89-97.

20. Kallel A, Bailon O, Carpentier AF .Clinical symptoms and symptomatic management of brain metastases. Bull Cancer 2011 Apr; 98(4):371-5.

21. Forsting M, Albert FK, Kunze S, Adams HP, Zenner D, Sartor K. Extirpation of glioblastomas : MR and CT follow-up of residual tumor and regrowth patterns. AJNR Am J Neuroradiol. 1993 Jan-Feb; 14(1):77-87.

22. Stummer W, Pichlmeier U, Meinel T, Wiestler OD, Zanella F, et al. (2006) Fluorescence-guided surgery with 5-aminolevulinic acid for resection of malignant glioma: a randomised controlled multicentre phase III trial. Lancet Oncol 7: 392-40.

23. Stupp R, Mason WP, van den Bent MJ, Weller M, Fisher B, Taphoorn MJB, et al. Radiotherapy plus concomitant and adjuvant temolozomide for glioblastoma. N Engl J Med.2005; 352(10):987-996.

24. Perry J, Chambers A, Spithoff K, Laperriere N. Gliadel wafers in the treatment of malignant glioma: a systematic review. Curr Oncol. 2007 Oct; 14 (5):189-94.

25. Hart MG, Grant R, garside R, Rogers G, Somerville M, Stein K. Chemotherapy wafers for high grade glioma.Cochrane Database Syst Rev. 2011 Mar 16; (3). 
26. Van Mieghem E, Wozniak A, Geussens Y, Menten J, De Vleeschouwer S, Van Calenbergh F, Sciot R, Van Gool S, Bechter OE, Demaerel P, Wilms G, Clement PM. Defining pseudoprogression in glioblastoma multiforme. Eur J Neurol. 2013 Oct; 20 (10):1335-41.

27. Knudsen-Baas KM, Moen G, Fluge $\varnothing$, Storstein A. Pseudoprogression in high-grade glioma. Acta Neurol Scand Suppl. 2013; (196):31-7.

28. Fink J, Born D, Chamberlain MC. Pseudoprogression: relevance with respect to treatment of high-grade gliomas. Curr Treat Options Oncol. 2011 Sep; 12 (3):240-52.

29. Brandsma D, Stalpers L, Taal W, Sminia P, van den Bent MJ. Clinical features, mechanisms, and management of pseudoprogression in malignant gliomas. Lancet Oncol 2008; 9(5):453-61.

30. Fabi A, Russillo M, Metro G, Vidiri A, Di Giovanni S, Cognetti F. Pseudoprogression and MGMT status in glioblastoma patients : implications in clinical practice. Anticancer Res. 2009 Jul; 29(7):2607-10.

31. Brandes AA, Franceschi E, Tosoni A, Blatt V, Pession A, Tallini G, et al. MGMT promoter methylation status scan predict the incidence and outcome of pseudoprogression after concomitant radiochemotherapy in newly diagnosed glioblastoma patients. J Clin Oncol. 2008; 26(13): 2192-7.

32. Curry RC, Dahiya S, Alva Venur V, Raizer JJ, Ahluwalia MS. Bevacizumab in high-grade gliomas: past, present and future. Expert Rev Anticanc Ther. 2015 Apr; 15 (4):387-97.

33. Ghiaseddin A, Peters KB.Use of bevacizumab in recurrent glioblastoma. CNS Oncol. 2015 May; 4(3):157-69. 
34. Wong ET, Gautam S, Malchow C, Lun M, Pan E, Brem S. Bevazicumab for recurrent glioblastoma multiforme : a meta-analysis.J Natl Compr Canc Netw. 2011 Apr; 9(4):403-7.

35. Ahluwalia MS, Vogelbaum MV, Chao ST, Mehta MM. Brain metastasis and treatment. F1000PrimeRep.2014 Dec 1; 6:114.

36. Al-Shamy G, Sawaya R. Management of brain metastases: the indispensable role of surgery. J. neurooncol. 2009; 92: 275-82.

37. Guillamo JS, Emery E, Busson A, Lechapt-Zalcman E, Constans JM, Defer GL. Current management of brain metastases. Rev Neurol (Paris). 2008 Jun-Jul; 164(6-7):560-8.

38. Bertolini F, Spallanzani A, Fontana A, Depenni R, Luppi G. brain metastases: an overview. CNS Oncol. 2015; 4(1):37-46.

39. Anocef référentiel sur les metastases cérébrales.

40. Dhermain F, Reyns N, Colin P, Metellus P, Mornex F, Noel G. Stereotactic radiotherapy in brain metastases. Cancer Radiotherap 2015 Feb; 19(1) :25-9.

41. Kim $\mathrm{CH}, \mathrm{Im}$ YS, Nam DH, Park K, Kim JH, Lee Jl.Gamma knife radiosurgery for ten or more brain metastases. J Korean Neurosurg Soc. 2008; 44:358-363.

42. Grandhi R, Kondziolka D, Panczykowski D, Monaco EA, III, Kano H, Niranjan A, Flickinger JC, Lunsford LD. Stereotactic radiosurgery using the Leksell Gamma Knife Perfexion unit in the management of patients with 10 or more brain metastases. J Neurosurg.2012; 117:237-245.

43. Bindal A.K., Bindal R.K., Hess K.R. Surgery versus radiosurgery in the treatment of brain metastasis. J. Neurosurg. 1996; 84 : 748-54. 
44. Parvez K, Parvez A, Zadeh G.The diagnosis and treatment of pseudoprogression, radiation necrosis and brain tumor recurrence. Int J Mol Sci. 2014 Jul 3; 15(7):11832-46.

45. Kumar AJ, Leeds NE, Fuller GN, Van Tassel P, Maor MH, Sawaya RE, et al. Malignant gliomas: MR imaging spectrum of radiation therapy and chemotherapy induced necrosis of the brain after treatment. Radiology 2000; 217(2):377-84.

46. Marks JE, Wong J.The risk of cerebral radionecrosis in relation to dose, time and fractionation. A follow-up study. Prog Exp Tumor Res 1986; 29:210-8.

47. Husain MM, Garcia JH. Cerebral « radiation necrosis: vascular and glial features. Acta Neuropathologica 1976; 7:243- 252.

48. Yoshii Y, Sugimoto K, Fujiwara K. Progressive enlargement of a mass lesion in late cerebral radionecrosis. J Clin Neurosci 2011; 18(6):853-5.

49. Castel JC, Caille JM. Imaging of irradiated brain tumours: value of magnetic resonance imaging. J. Neuroradiol 1989; 16:81-132.

50. Sawaya R.The fibrinolytic enzymes in the biology of brain tumors. In: Sawaya MD, ed. Fibrinolysis and the central nervous system. Philadelphia, Pa: Hanley \& Belfus, 1990; 106126.

51. Wong ST, Loo KT, Yam KY, Hung WM, Fok KF, Yuen SC, Fong D. Results of excision of cerebral radionecrosis: experience in patients treated with radiation therapy for nasopharyngeal carcinoma. J Neurosurg. 2010 Aug; 113(2):293-300. 
52. Lee AWM, Kwong DLW, Leung SF, Tung SY, Sze WM, Sham JST, et al. Factors affecting risk of symptomatic temporal lobe necrosis: significance of fractional dose and treatment time. Int J Radiat Oncol Biol Phys 2002; 53(1):75-85.

53. Ruben JD, Dally M. Bailey M, Smith R, McLean CA, Fedele P. Cerebral radiation necrosis: incidence, outcomes, and risk factors with emphasis on radiation parameters and chemotherapy. Int J Radiat Oncol Biol Phys 2006; 65(2):499-508.

54. Blonigen BJ, Steinmetz RD, Levin L, Lamba MA, Warnick RE, Breneman JC.Irradiated volume as a predictor of brain radionecrosis after linear accelerator stereotactic radiosurgery. Int J Radiat Oncol Biol Phys 2006; 64(2):419-24.

55. Korytko T, Radivoyevitch T, Colussi V, Wessels B, Pillai K, Maciunas R, et al. 12Gy gamma knife radiosurgical volume is a predictor for radiation necrosis in non -AVM intracranial tumors. Int J Radiat Oncol Biol Phys 2006; 64(2):419-24.

56. Forsyth PA, Kelly PJ, Cascino TL, Scheithauer BW, Shaw EG, Dinapoli RP, Atkinson EJ. Radiation necrosis or glioma recurrence: is computer- assisted stereotactic biopsy useful? J Neurosurg. 1995 Mar; 85(2) : 436-44.

57. Leber KA, Eder HG, Kovac H, Anegg U, Pendl G. Treatment of cerebral radionecrosis by hyperbaric oxygen therapy. Stereotact Funct Neurosurg. 1998 Oct; 70 Suppl 1:229-36.

58. Chao ST, Ahluwalia MS, Barnett GH, Stevens GH, Murphy ES, Stockham AS, Shiue K, Suh JH.Challenges with the diagnosis and treatment of cerebral radiation necrosis. Int J Radiat Oncol Biol Phys. 2013 Nov 1; 87(3):449-57. 
59. Tye K, Engelhard HH, Slavin KV, Nicholas MK, Chmura SJ, Kwok Y, Ho DS, Weichselbaum RR, Koshy M. An analysis of radiation necrosis of the central nervous system treated with bevacizumab. J Neurooncol 2014 Apr; 117(2):321-7.

60. Gronier S, Bourg V, Frenay M, Cohen M, Mondot L, Thomas P, Lebrun C. Bevacizumab for the treatment of cerebral radionecrosis. Rev Neurol (Paris). 2011 Apr; 167(4) :331-6.

61. Nonoguchi N, Miyatake S, Fukumoto M, Furuse M, Hiramatsu R, Kawabata S, Kuroiwa T, Tsuji M, Fukumoto M, Ono K. The distribution of vascular endothelial growth factorproducing cells in clinical radiation necrosis of the brain: pathological consideration of their potential roles. J Neurooncol. 2011 Nov; 105(2):423-31.

62. Guillevin R, Menuel C, Vallée JN.Multimodal magnetic resonance imaging of brain tumors. Rev Neurol. 2011 Oct; 167(10):70 4-14.

63. Grosu AL, Feldmann H, Dick S, Dzewas B, Nieder C, Gumprecht H, Frank A, Schwaiger M, Molls M, Weber WA. Implications of IMT-SPECT for postoperative radiotherapy planning in patients with gliomas .Int J Radiat Oncol Biol Phys. 2002 Nov 1; 54(3):842-54.

64. Mullins ME, Barest GD, Schaefer PW, Hochberg FH, Gonzalez RG, Lev MH. Radiation necrosis versus glioma recurrence: conventional MR imaging clues to diagnosis. AJNR Am J Neuroradiol. 2005 Sep; 26(8): 1967-72.

65. Reddy K, Westerly D, Chen C. MRI patterns of T1 enhancing radiation necrosis versus tumour recurrence in high-grade gliomas. J Med Imaging Radiat Oncol 2013; 57(3):349-55.

66. Huang J, Wang AM, Shetty A, Maitz AH, Yan D, Doyle D, et al. Differentiation between intra-axial metastatic tumor progression and radiation injury following fractionated radiation 
therapy or stereotactic radiosurgery using MR spectroscopy, perfusion MR imaging or volume progression modeling. Magn Reson Imaging 2011; 29(7):993-1001.

67. Patel TR, McHugh BJ, Bi WL, Minja FJ, Knisely JPS, Chiang VL. A comprehensive review of MR imaging changes following radiosurgery to 500 brain metastases. AJNR Am J Neuroradiol 2011; 32(10):1885-92.

68. Dequesada IM, Quisling RG, Yachnis A, Friedman WA. Can standard magnetic resonance imaging reliably distinguish recurrent tumor from radiation necrosis after radiosurgery for brain metastases? A radiographic-pathological study. Neurosurgery 2008; 63(5) :898-904.

69. Stockhalm AL, Tievsky AL, Koyfman SA, Reddy CA, Suh JH, Vogelbaum MA, et al. Conventional MRI does not reliably distinguish radiation necrosis from tumor recurrence after stereotactic radiosurgery. J Neurooncol 2012; 109(1):149-58.

70. Kano H, Kondziolka D, Lobato-Polo J, Zorro O, Flickinger JC, Lunsford LD. T1/T2 matching to differentiate tumor growth from radiation effects after stereotactic radiosurgery. Neurosurgery 2010; 66(3):48691.

71. Leeman JE, Clump DA, Flickinger JC, Mintz AH, Burton SA, Heron DE. Extent of perilesional oedema differentiates radionecrosis from tumor recurrence following stereotactic radiosurgery for brain metastases. Neuro Oncol 2013; 15(12):1732-38.

72. Koh DM, Collins DJ. Diffusion-weighted MRI in the body: applications and challenges in oncology. AJR Am J Roentgenol. 2007 Jun; 188(6):1622-35. 
73. Raimbault A, Cazals X, Lauvin MA, Destrieux C, Chapet S, Cottier JP. Radionecrosis of malignant glioma and cerebral metastasis: a diagnostic challenge in MRI. Diag Interv Imaging. 2014 Oct; 95(10):958-1000.

74. Asao C, Korogi Y, Kitajima M, Hirai T, Baba Y, Makino K, et al.Diffusion-weighted imaging of radiation-induced brain injury for differentiation rom tumor recurrence. Am J Neuroradiol 2005; 26(6):1455-60.

75. Hein PA, Eskey CJ, Dunn JF, Hug EB. Diffusion-weighted imaging in the follow-up of treated high-grade gliomas: tumor recurrence versus radiation injury. AJNR Am J Neuroradiol 2004; 25(2):201-9.

76. Bobek-Billewicz B, Stasik-Pres G, Majchrzak H, Zarudzki L. Differentiation between brain tumor recurrence and radiation injury using perfusion, diffusion-weighted imaging and MR spectroscopy. Folia Neuropathol 2010; 48(2):81-92.

77. Chan Y, Yeung DKW, Leung S, Chan P. Diffusion weighted-magnetic resonance imaging in radiation-induced cerebral necrosis.Apparent diffusion coefficient in lesion components. J Comput Assist Tomogr 2003; 27(5):674-80.

78. Cuenod CA, Balvay D. Perfusion and vascular permeability: basic concepts and measurement in DCE-CT and DCE-MRI. Diagn Interv Imaging. 2013 Dec; 94(12):1187-204.

79. Griffith B, Jain R. Perfusion Imaging in Neuro-Oncology: Basic Techniques and Clinical Applications. Radiol Clin North Am 2015 May; 53(3):497-511.

80. Lacerda S, Law M. Magnetic resonance perfusion and permeability imaging in brain tumors. Neuroiamging Clin N Am. 2009 Nov; 19(4):527-57. 
81. Villringer A, Rosen BR, Belliveau JW, Ackerman JL, Lauffer RB, Buxton RB, et al. Dynamic imaging with lanthanide chelates in normal brain: contrast due to magnetic susceptibility effects. Magn Reson Med 1988; 6: 164-74.

82. Grand S, Tahon F, Attye A, Lefournier V, Le Bas JF, Krainik A. Perfusion imaging in brain disease. Diagn Interv Imaging. 2013 Dec; 94(12):1241-57.

83. Le Bas JF, Grand S, Krainik A, Lefournier V, Tropres I, Rémy C. Perfusion MR imaging in brain tumors. J Radiol. 2006 Jun; 87(6Pt2):807-21.

84. Hu LS, Baxter LC, Smith KA, Feuerstein BG, Karis JP, Eschbacher JM, Coons SW, Nakaji $P$, yeh RF, Debbins J, Heiserman JE. Relative cerebral blood volume values to differentiate high-grade glioma recurrence from posttreatment radiation effect: direct correlation between image-guided tissue histopathology and localized dynamic susceptbility-weighted contrast-enhanced perfusion MR imaging measurements. AJNR Am J Neuroradiol 2009 Mar; 30(3); 552-8.

85. Nieder C, Andratschke N, Price RE, Rivera B, Ang KK.Innovative prevention strategies for radiation necrosis of the central nervous system. Anticancer Res 2002; 22(2A):1017-1023.

86. Wetzel SG, Cha S, Johnson G et al. Relative cerebral blood volume measurements in intracranial mass lesions: interobserver and intraobserver reproductibility study. Radiology $2002 ; 224: 797-803$.

87. Barajas RF, Chang JS, Segal MR, Parsa AT, McDermott MW, Berger MS, et al. Differentiation of recurrent glioblastoma multiform from radiation necrosis after external beam radiation therapy with dynamic susceptibility-weighted contrast-enhanced perfusion MR imaging. Radiology 2009; 253(2):486-96. 
88. Barajas RE, Chang JS, Sneed PK, Segal MRn McDermott MW, Cha S. Distinguishing recurrent intra-axial metastatic tumor from radiation necrosis gamma knife radiosurgery using dynamic susceptibility-weighted contrast-enhanced perfusion MR imaging. AJNR Am J Neuroradiol. 2009 Feb; 30(2):367-72].

89. Cha S, Lupo JM, Chen MH, et al. Differentiation of gliobastoma multiforme and single brain metastasis by peak height and percentage of signal intensity recovery derived from dynamic susceptibility-weighted contrast-enhanced perfusion MR imaging. AJNR Am J Neuroradiol 2007; 28:1078-84.

90. Cha S, Lu S, Johnson G, et al. Dynamic susceptibility contrast MR imaging: correlation of signal intensity changes with cerebral blood volume measurements. J Magn Reson Imaging 2000; 11:114-19.

91. Le Bars E, Gondry-Jouet C, Deramond H, Le Gars D, Idy-Peretti I. MR diffusion and perfusion imaging in clinical practice. J Neuroradiol 2000 Mar; 27(1):39-51.

92. Paulson ES, Schmainda KM. Comparison of dynamic susceptibility -weighted contrast-enhanced MR methods: recommendations for measuring relative cerebral blood volume in brain tumors. Radiology 2008 Nov; 249(2):601-13.

93. Kitahara S, Nakasu S, Murata K, Sho K, Ito R. Evaluation of treatment-induced cerebral white matter injury by using diffusion tensor MR imaging : initial experience. AJNR Am J Neuroradiol 2005; 26(9):2200-6.

94. Shin KE, Ahn KJ, Choi HS, Jung SL, Kim BS, Jeon SS, Hong YG. DCE and DSC MR perfusion imaging in the differentiation of recurrent tumour from treatment-related changes in patients with glioma. Clin Radiol 2014 Jun; 69(6): e264-72. 
95. Grand S, Tropres I, Hoffmann D, Ziegler A, Le Bas JF.Proton magnetic resonance spectroscopy (1H-MRS) for the diagnosis of brain tumors and the evaluation of treatment. Neurochirurgie. 2005 Sep; 51(3-4pt2):299-308.

96. Elias AE, Carlos RC, Smith EA, Frechtling D, George B, Maly P, Sundgren PC.MR spectroscopy using normalized and non-normalized metabolite ratios for differentiating recurrent brain tumor from radiation injury. Acad Radiol. 2011; 18(9):1101-8.

97. Graves EE, Nelson SJ, Vigneron DB, et al.Serial proton MR spectroscopic imaging of recurrent malignant gliomas after gamma knife radiosurgery. Am J Neuroradiol 2001; 22: 613-24.

98. Heesters MA, Kamman RL, Mooyaart EL, Go KG. Localized proton spectroscopy of inoperable brain gliomas Response to radiation therapy J Neurooncol 1993 Jul; 17(1):27-35.

99. Smith EA, Carlos RC, Junck LR, Tsien Cl, Elias A, Sundgren PC.Developing a clinical decision model: MR spectroscopy to differentiate between recurrent tumor and radiation change in patients with new contrast-enhancing lesions. AJR Am J Roentgenol 2009; 192(2):W45-52.

100. Yeung DK, Chan Y, Leung S, Poon PM, Pang C. Detection of an intense resonance at $2.4 \mathrm{ppm}$ in $1 \mathrm{H}$ MR spectra of patients with severe late-delayed, radiation-induced brain injuries. Magn Reson Med. 2001 Jun; 45(6):994-1000.

101. Herminghaus S, Pilatus U, Moller-Hartmann W, et al. Increased choline levels coincide with enhanced proliferative activity of human neuroepithelial brain tumors.NMR Biomed. 2002 Oct; 15(6):385-92. 
102. Schlemmer HP, Bachert P, Herfarth K et al. Proton MR spectroscopic evaluation of suspicious brain lesions after stereotactic radiotherapy. AJNR Am J Neuroradiol.2001; 22:1316-24.

103. Kickingereder P, Dorn F, Blau T, Schmidt M, Kocher $M$, Galldiks $N$, et al. Differentiation of local tumor recurrence from radiation-induced changes after stereotactic radiosurgery for treatment of brain metastasis: case report and review of the literature. Radiat Oncol. 2013; 8:52.

104. Van Tassel P, Bruner JM, Maor MH et al.MR of toxic effects of accelerated fractionation radiation therapy and carboplatin chemotherapy for malignant gliomas. Am J Neuroradiol 1995; 16:715-726.

105. Zhang $\mathrm{H}, \mathrm{Ma} \mathrm{L}, \mathrm{Wu} \mathrm{C}, \mathrm{Xu}$ BN.Performance of SPECT in the differential diagnosis of glioma recurrence from radiation necrosis. J Clin Neurosci. 2015 Feb; 22(2): 229-37.

106. Le Jeune FP, Dubois F, Blond S, Steinling M. Sestamibi technetium-99 m brain singlephoton emission computed tomography to identify recurrent glioma in adults: 201 studies. J Neurooncol. 2006 Apr; 77(2):177-83.

107. Matsunaga S, Shuto T, Takase H, Ohtake M, Tomura N, Tanaka T, Sonoda M. Semiquantitative analysis using thallium-201 SPECT for differential diagnosis between tumor recurrence and radiation necrosis after gamma knife surgery for malignant brain tumors. Int J Radiat Oncol Biol Phys. 2013 Jan 1; 85(1):47-52.

108. Foehrenbach H, Albérini JL, Maszelin P, Bonardel G, Tenenbaum F, de Dreuille O, Richard B, Gaillard JF, Devaux JY. Positron emission tomography in clinical oncology. Presse Med. 2003 Feb 15; 32(6):276-83. 
109. Bonardel G, Lecoules S, Mantzarides M, Carmoi T, Gontier E, Blade JS, Soret M, Foehrenbach H, Algayres JP.Positron emission tomography in internal medicine. Epub 2007 Jun 26.

110. Warburg O. Origin of cancer cells. Oncologia. 1956; 9(2):75-83.

111. Vaylet F, Margery J, Bonardel G, Le Floch H, Rivière F, Gontier E, Ngampolo I, Mairovitz A, Marotel C, Foehrenbach $\mathrm{H}$. What is the role of FDG-PET in thoracic oncology in 2010? Rev Pneumol Clin. 2010 Sep; 66(4):221-38.

112. Horky LL, Hsiao EM, Weiss SE, Drappatz J, Gerbaudo VH. Dual phase FDG-PET imaging of brain metastases provides superior assessment of recurrence versus post-treatment necrosis. J Neuroroncol 2011; 103:137-46.

113. Langleben DD, Segall GM.PET in differentiation of recurrent brain tumor from radiation injury. J Nucl Med. 2000 Nov; 41(11):1861-7.

114. Chao ST, Suh JH, Raja S, Lee SY, Barnett G. The sensitivity and specificity of FDG PET in distinguishing recurrent brain tumor from radionecrosis in patients treated with stereotactic radiosurgery. Int J Cancer. 2001 Jun 20; 96(3):191-7.

115. Talbot JN, Kerrou K, Gault N, Gutman F, Grahek D, Touboul E, Schlienger M, Montravers F. PET and malignant cerebral tumors.Presse Med. 2006; 35:1347-53.

116. Caroline I, Rosenthal MA. Imaging modalities in high-grade gliomas: pseudoprogression, recurrence, or necrosis? J Clin Neurosci. 2012 May; 19(5):633-7. 
117. Patsouris A, Augereau P, Tanguy JY, Morel O, Menei P, Rousseau A, Paumier A. Differential diagnosis of local tumor recurrence or radionecrosis after stereotactic radiosurgery for treatment of brain metastasis.Cancer Radiother. 2014 Mar; 18(2):142-6.

118. Glaudemans AW, Enting RH, Heesters MA, Dierckx RA, van Rheenen RW, Walenkamp AM, Slart RH.Value of 11C-methionine PET in imaging brain tumours and metastases. Eur J Nucl Med Mol Imaging 2013 Apr; 40(4):615-35.

119. Singhal T, Narayanan TK, Jain V, Mukherjee J, Mantil J. 11C-L -methionine positron emission tomography in the clinical management of cerebral gliomas. Mol Imaging Biol. 2008Jan-Feb; 10(1):1-18.

120. Götz I, Grosu AL [(18F)] FET-PET imaging for Treatment and Response Monitoring of Radiation Therapy in Malignant Glioma Patients-A Review. Front Oncol. 2013 Apr 25; 3:104.

121. Takenaka S, Asano Y, Shinoda J, Nomura Y, Yonezawa S, Miwa K, Yano H, Iwama T.Comparison of(11) C-methionine, (11) C-choline, and (18) F-fluorodeoxyglucose-PET for distinguishing glioma recurrence from radiation necrosis. Neurol Med Chir (Tokyo). 2014; 54(4):280-9.

122. Weber WA, Wester HJ, Grosu AL, Herz M, Dzewas B, Feldmann HJ, et al.O-(2-[18F] fluoroethyl)-L-tyrosine and L-[methyl-11C] methionine uptake in brain tumours: initial results of a comparative study. Eur J Nucl Med 2000; 27:542-9.

123. Pöpperl G, Götz C, Rachinger W, Gildehaus FJ, Tonn JC, Tatsch K. Value of O-(2-[18F] fluoroethyl)-L-tyrosine PET for the diagnosis of recurrent glioma.Eur J Nucl Med Mol Imaging. 2004 Nov; 31(11):1464-70. 
124. Nataf V, Kerrou K, Balogova S, Pene F, Huchet V, Gutman F, Prignon A, Muresan IP, Giannesini C, Izrael V, Schlienger M, Talbot JN. Fluroethyltyrosine $18 \mathrm{~F}$ PET in the detection of brain tumours. Bull Cancer. 2010 May; 97(5):495-506.

125. Galldiks N, Stoffels G, Filss C, Rapp M, Blau T, Tscherpel C, Ceccon G, Dunkl V, Weinzierl M, Stoffel M, Sabel M, Fink GR, Shah NJ, Langen KJ.The use of dynamic O-(2-18-Ffluoroethyl)-I-tyrosine PET in the diagnosis of patients with progressive and recurrent glioma. Neuro Oncol.2015May 24.

126. Chen W, Silverman DH, Delaloye S, Czernin J, Kamdar N, Pope W, Satyamurthy N, Schiepers C, Cloughesy T. 18 F-DOPA PET imaging of brain tumors: comparison study with 18F- FDG PET and evaluation of diagnostic accuracy. J Nucl Med. 2006 Jun; 47(6) :904-11.

127. Becherer A, Karanikas G, Szabo M, Zettinig G, Asenbaum S, Marosi C, Henk C, Wunderbaldinger P, Czech T, Wadsak W, Kletter K. Brain tumour imaging with PET : a comparison between (18F] fluorodopa and [11C] methionine.Eur J Nucl Med Mol Imaging. 2003 Nov; 30(11):1561-7.

128. Lizarraga KJ, Allen -Auerbach M, Czernin J, DeSalles AA, Yong WH, Phelps ME, Chen W. (18) F-F DOPA PET for differentiating recurrent or progressive brain metastatic tumors from late or delayed radiation injury after radiation treatment. J Nucl Med. 2014 Jan; 55(1):30-6.

129. Lizagarra KJ, De Salles AA, Chen W. 18F-fluorodopa positron -emission tomography: an emerging imaging modality for patients with brain metastases.Expert Rev Med Devices. 2014 Jul; 11(4):327-9. 
130. Cicone F, Minniti G, Romano A, Papa A, Scaringi C, Tavanti F, Bozzao A, Maurizi Enrici R, Scopinaro F. Accuracy of F-DOPA PET and perfusion -MRI for differentiating radionecrotic from progressive brain metastases after radiosurgery. Eur J Nucl Med Mol Imaging.2015 Jan; 42(1):103-11.

131. Karunanithi S, Sharma P, Kumar A, Khangembam BC, Bandopadhyaya GP, Kumar R, Goenka A, Gupta DK, Malhotra A, Bal C. Comparative diagnostic accuracy of contrastenhanced MRI and (18F) F-F DOPA PET-CT in recurrent glioma. Eur Radiol. 2013 Sep; 23(9): 2628-35.

132. Sugahara $T$, Korogi $Y$, Tomiguchi S, Shigematsu $Y$, Ikushima I, Kira $T$, et al. Posttherapeutic intra-axial brain tumor: the value of perfusion-sensitive contrast-enhanced MR imaging for differentiating tumor recurrence from nonneoplastic contrast-enhancing tissue. AJNR Am J Neuroradiol 2000; 21(5): 901-5.

133. Kim HS, Goh MJ, Kim N, Choi CG, Kim SJ, Kim JH. Which combination of MR imaging modalities is best for predicting recurrent glioblastoma? Study of diagnostic accuracy and reproductibility.Radiology. 2014 Dec; 273(3):831-43.

134. Blasel S, Zagorcic A, Jurcoane A, Bähr O, Wagner M, Harter PN, Hattingen E. Perfusion MRI in the Evaluation of Suspected Glioblastoma Recurrence. J Neuroimaging.2015 Apr 24.

135. Andre JB, Nagpal S, Hippe DS, Ravanpay AC, Schmiedeskamp H, Bammer R, Palagallo GJ, Recht L, Zaharchuk G. Cerebral Blood Flow Changes in Glioblastoma Patients Undergoing Bevacizumab Treatment Are Seen in Both Tumor and Normal Brain. Neuroradiol J. 2015 Apr; 28(2):112-9. 
136. Metellus P, Dutertre G, Mekkaoui C, Nanni I, Fuentes S, Ait-Ameur, et al.Value of relative cerebral blood volume measurement using perfusion MRI in glioma management. Neurochirurgie 2008; 54(4):503-11.

137. Bisdas $S$, Naegele $T$, Ritz R, et al.Distinguishing recurrent high-grade gliomas from radiation injury: a pilot study using dynamic contrast-enhanced MR imaging. Acad Radiol $2011 ; 18: 575-83$.

138. Prager AJ, Martinez N, Beal K, Omuro A, Zhang Z, Young RJ.Diffusion and perfusionMRI to differentiate treatment-related changes including pseudoprogression from recurrent tumors in high-grade gliomas with histopathologic evidence. AJNR Am J Neuroradiol. 2015 May; 36(5):877-85.

139. Zhang H, Ma L, Shu C, Wang YB, Dong LQ.Diagnostic accuracy of diffusion MRI with quantitative $A D C$ measurements in differentiating glioma recurrence from radiation necrosis. J Neurol Sci. 2015 Apr 15; 351(1-2):65-71.

140. Zhang $\mathrm{H}, \mathrm{Ma} \mathrm{L}$, Wang $\mathrm{Q}$, Zheng $\mathrm{X}, \mathrm{Wu} \mathrm{C}, \mathrm{Xu} B N$. Role of magnetic resonance spectroscopy for the differentiation of recurrent glioma from radiation necrosis: a systematic review and meta-analysis. Eur J Radiol. 2014 Dec; 83(12):2181-9.

141. Rock JP, Scarpace L, Hearshen D, Gutierrez J, Fisher JL, Rosenblum M, Mikkelsen T. Associations among magnetic resonance spectroscopy, apparent diffusion coefficients, and image-guided histopathology with special attention to radiation necrosis. Neurosurgery. 2004 May; 54(5):1111-7. 
142. Ellingson BM, Lai A, Nguyen HN, Nghiemphu PL, Pope WB, Cloughesy TF. Quantification of nonenhancing tumor burden in gliomas using effective T2 maps derived from dual-echo turbo spin-echo MRI. Clin Cancer Res. 2015 Apr 21.

143. Thompson EM, Dosa E, Kraemer DF, Neuwelt EA. Correlation of MRI sequences to assess progressive glioblastoma multiforme treated with bevacizumab. J Neurononcol. 2011 Jun; 103(2):353-60.

144. Calabria F, Cascini GL. Current status of 18F-DOPA PET imaging in the detection of brain tumor recurrence. Hell J Nucl Med. 2015 Jul 20.

145. Youland RS, Kitange GJ, Peterson TE, Pafundi DH, Ramiscal JA, Pokorny JL, Giannini C, Laack NN, Parney IF, Lowe VJ, Brinkmann DH, Sarkaria JN.The role of LAT 1 in (18F)-DOPA uptake in malignant gliomas. J Neurooncol. 2013 Jan; 111(1):11-8.

146. Wiriyasermkul P, Nagamori S., et al. Transport of 3-fluoro-L-alpha-methyl-tyrosine by tumor-upregulated L-type amino acid transporter 1: a cause of the tumor uptake in PET. J Nucl Med. 2012 Aug; 53(8):1253-61.

147. Caroline Papin-Michault. Etude de l'expression de LAT1 dans les métastases cérébrales.Human health and Pathology. 2013

148. Darcourt J, Dufour $\mathrm{M}$, et al. 18F-DOPA PET for the diagnosis of brain tumor recurrence: Correlation to LAT 1 expression. J Nuclear Med. 2014; 55(Supplement 1):1889.

149. Young RJ, Gupta A, Shah AD, Graber JJ, Chan TA, Zhang Z, Shi W, Beal K, Omuro AM.MRI perfusion in determining pseudoprogression in patients with gliobastoma. Clin. Imaging. 2013 Jan-Feb; 37(1):41-9. 


\section{8- ANNEXE}

\begin{tabular}{|c|c|c|c|}
\hline REPONSE & $\begin{array}{l}\text { CRITERES } \\
\text { CLINIQUES }\end{array}$ & $\begin{array}{l}\text { CRITERES } \\
\text { IRM }\end{array}$ & REMARQUES \\
\hline $\begin{array}{l}\text { Réponse } \\
\text { Complète } \\
\text { (RC) }\end{array}$ & $\begin{array}{l}\text { Demande tous les critères } \\
\text { suivants: } \\
\text { * amélioration ou } \\
\text { stabilité clinique } \\
\text { * sevrage complet des } \\
\text { corticoides (sauf } \\
\text { posologie de } \\
\text { substitution) }\end{array}$ & $\begin{array}{l}\text { * Disparition complète des lésions } \\
\text { mesurables et non mesurables } \\
\text { prenant le contraste }(\mathrm{T} 1 \text { gadolinium) } \\
\text { pendant au moins } 4 \text { semaines } \\
\text { * Diminution ou stabilité du signal } \\
\text { T2/FLAIR } \\
\text { * Pas de nouvelles lésions }\end{array}$ & $\begin{array}{l}\text { Les patients } \\
\text { n'ayant que des } \\
\text { lésions non } \\
\text { mesurables ne } \\
\text { peuvent pas être } \\
\text { classés en RC mai } \\
\text { simplement en } \\
\text { stabilisation }\end{array}$ \\
\hline $\begin{array}{l}\text { Réponse } \\
\text { Partielle } \\
\text { (RP) }\end{array}$ & $\begin{array}{l}\text { Demande tous les critères } \\
\text { suivants: } \\
\text { * amélioration ou } \\
\text { stabilité clinique } \\
\text { * dose stable ou } \\
\text { diminuée des } \\
\text { corticoides }\end{array}$ & $\begin{array}{l}\text { * Diminution de } 50 \% \text { ou plus de la } \\
\text { somme des produits des diamètres } \\
\text { perpendiculaires des lésions prenant } \\
\text { le contraste par rapport à l'examen } \\
\text { de référence } \\
\text { * Pas de nouvelles lésions mesurables } \\
\text { ou non mesurables } \\
\text { * Diminution ou stabilité du signal } \\
\text { T2/FLAIR sous une dose stable ou } \\
\text { diminuée de corticoïdes }\end{array}$ & $\begin{array}{c}\text { Les patients } \\
\text { n'ayant que des } \\
\text { lésions non } \\
\text { mesurables ne } \\
\text { peuvent pas être } \\
\text { classés en RC maí } \\
\text { simplement en } \\
\text { stabilisation }\end{array}$ \\
\hline Stabilité & $\begin{array}{l}\text { Demande tous les critères } \\
\text { suivants: } \\
\text { * absence de réponse } \\
\text { complète, partielle } \\
\text { ou de progression } \\
\text { * dose stable ou } \\
\text { diminuée des } \\
\text { corticoïdes }\end{array}$ & $\begin{array}{l}\text { * Diminution ou stabilité du signal } \\
\text { T2/FLAIR sous une dose stable ou } \\
\text { diminuée de corticoïdes }\end{array}$ & \\
\hline Progression & $\begin{array}{l}\text { * Détérioration } \\
\text { clinique non } \\
\text { attribuable à une } \\
\text { autre cause que la } \\
\text { tumeur (épilepsie, } \\
\text { effets adverses de } \\
\text { médicaments, } \\
\text { complication des } \\
\text { traitements, accident } \\
\text { vasculaire, infection) } \\
\text { * augmentation de la } \\
\text { dose des corticoïdes } \\
\text { rendue nécessaire par } \\
\text { la détérioration } \\
\text { clinique }\end{array}$ & $\begin{array}{c}\text { *Augmentation de } 25 \% \text { ou plus de la } \\
\text { somme des produits des diamètres } \\
\text { perpendiculaires des lésions prenant } \\
\text { le contraste par rapport à l'examen } \\
\text { ayant mesuré les dimensions } \\
\text { tumorales les plus faibles (soit avant } \\
\text { le traitement, soit au moment de la } \\
\text { meilleure réponse) } \\
\text { * augmentation du signal T2/FLAIR } \\
\text { non lié à une comorbidité : } \\
\text { irradiation, démyélinisation, accident } \\
\text { ischémique, infection, épilepsie, } \\
\text { modifications post-opératoires, autres } \\
\text { effets des traitements. } \\
\text { * Toutes nouvelles lésions mesurables } \\
\text { ou non mesurables } \\
\text { * Absence de nouvelle évaluation IRM } \\
\text { liée au décès ou à la détérioration } \\
\text { clinique }\end{array}$ & \\
\hline
\end{tabular}

\section{Critères RANO (ANOCEF)}




\section{9 - TABLE DES ILLUSTRATIONS}

\section{$\underline{\text { Index des tableaux }}$}

Tableau 1: Caractéristiques de la population

page 113

Tableau 2: Concordance et performances diagnostiques de I'IRM multimodale par rapport au gold-standard

page 115

Tableau 3 : Evaluation des données morphologiques

page 116

Tableau 4 : Mesures de paramètres quantitatifs par imagerie de perfusion T2*

page 117

Tableau 5: $\quad$ Mesure de paramètres quantitatifs par imagerie de perfusion T1

page 120

Tableau 6: Concordance et performances diagnostiques de la TEP-TDM à la ${ }^{18}$ F-FDOPA par rapport au gold-standard

page 128 


\section{Index des figures}

Figure 1: Classification OMS des tumeurs du système nerveux central page 16

Figure 2: Anatomie du système nerveux central

page 17

Figure 3: $\quad$ Principaux types de gliomes selon la classification de l'OMS 2007 page 20

Figure 4: Grading de la classification OMS des gliomes

page 21

Figure 5: Grading des oligodendrogliomes de l'Hôpital Saint-Anne

page 23

Figure 6: Protocole Stupp

page 31

Figure 7: $\quad$ Aspect anatomo-pathologique observé en cas de radiolésion

page 42

Figure 8: Courbe de premier passage en imagerie de perfusion $\mathrm{T}^{*}$

page 54

Figure 9: $\quad$ Méthode de calcul des paramètres PSR et HP selon Barajas page 60

Figure 10: Signification des parties de la courbe de rehaussement tissulaire en imagerie de perfusion T1

page 62

Figure 11: Post-traitement des séquences de perfusion T1 à l'aide du logiciel GEN IQ page 89

Figure 12: Post-traitement en imagerie de perfusion T2*à l'aide du mode Brainstat AIF page 91

Figure 13: Post-traitement en imagerie de diffusion page 92

Figure 14: Pattern de rehaussement de type nodulaire ou annulaire page 96 
Figure 24: Spectre montrant la présence d'un pic de lipides et d'une élévation du pic de choline page 104

Figure 25: $\quad$ TEP-TDM à la ${ }^{18} \mathrm{~F}-\mathrm{FDOPA}$ page 105

Figure 26: Répartition des types histologiques dans la population globale page 109

Figure 27: Répartition des tumeurs gliales et des métastases cérébrales dans la population page 110

Figure 28: Modalités du diagnostic final page 111

Figure 29 : Représentation des paramètres mesurés en imagerie de perfusion $\mathrm{T}^{*}$ sous forme de boîtes à moustache page 118 
Figure 30: Représentation des paramètres mesurés en imagerie de perfusion T1 sous forme de boîtes à moustache page 121

Figure 31: Courbes ROC correspondant aux performances diagnostiques des paramètres en imagerie de perfusion page 124

Figure 32: Diagrammes de Bland Altman représentant la reproductibilité des mesures entre les lecteurs junior et senior page 127

Figure 33: Radionécrose histologiquement prouvée d’une métastase cérébrale secondaire à un primitif d'origine pulmonaire page 130

Figure 34: Autre cas de radionécrose histologiquement prouvée d'une métastase cérébrale secondaire à un primitif d'origine pulmonaire page 131

Figure 35: Métastase cérébrale d'un primitif d'origine pulmonaire page 132

Figure 36 : Forme hyper-vasculaire de métastase cérébrale observée dans notre série page 136

Figure 37: Absence de diagnostic précoce des deux modalités d'examen page 145 


\section{0 - LISTE DES ABREVIATIONS}

ADC

AIF

AMM

ASL

BOLD

Cho

$\mathrm{CHU}$

$\mathrm{Cr}$

DCE-MR

DSC-MRI

${ }^{18}$ F-FDG

${ }^{18}$ F-FDOPA $\quad 6-\left[{ }^{18} \mathrm{~F}\right]$-fluoro-dihydroxy-L-phenylalanine

${ }^{18}$ F-FET $\quad\left[{ }^{18} \mathrm{~F}\right]$-Fluoro-L-Tyrosine

FLAIR Fluid Attenuated Inversion Recovery

GLUT GLUcose Transporter

HP

Hauteur du Pic 
IAUGC Initial Area Under the Gadolinium concentration Curve

IRM Imagerie par Résonance Magnétique

LAT 1 Large Amino-Acid Transporter

MET $\quad{ }^{11}$ C-Methionine

MGMT Méthyl-Guanine-Méthyl-Transférase

N Radionécrose

NAA N-Acétyl-Aspartate

OMS Organisation Mondiale de la Santé

P Progression tumorale

PSR Pourcentage de Récupération du Signal

RCP Réunion de Concertation Pluridisciplinaire

RHCS Radiothérapie Hypo-fractionnée en Conditions Stéréotaxiques

RMCS Radiothérapie Mono-fractionnée en Conditions Stéréotaxiques

ROC Receiver Operating Characteristic

ROI Region of Interest

SNC Système Nerveux Central

SUV Standardized Uptake Value

TE Temps d'écho 
TEMP Tomographie par Emission MonoPhotonique

TEP Tomographie par Emission de Positons

TI Temps d'Inversion

TPSNC Tumeur Primitive du Système Nerveux Central

TR Temps de Répétition

VEGF Vascular Endothelial Growth Factor

VIF Vessel Input Function

VSC Volume Sanguin Cérébral 


\section{1 - TABLE DES MATIERES}

1- INTRODUCTION

2- RAPPELS

2.1 Généralités sur les tumeurs cérébrales page 13

page 15

page 15

2.1.1 Historique et classification histologique OMS des tumeurs du système nerveux central

2.1.2 Les tumeurs primitives du système nerveux central (TPSNC) : rappels anatomiques et histologiques. L'exemple des tumeurs gliales

page 17

2.1.2.1. Epidémiologie et facteurs de risque de tumeurs gliales page 19

$\begin{array}{ll}\text { 2.1.2.2. Grading des tumeurs gliales } & \text { page } 20\end{array}$

2.1.2.3. Biologie moléculaire page 24

2.1.3 Les tumeurs secondaires du système nerveux central page 26

2.2 Prise en charge thérapeutique des tumeurs cérébrales page 28

2.2.1 Traitement symptomatique des tumeurs cérébrales page 28

2.2.2 Traitements spécifiques des tumeurs gliales malignes page 29

2.2.1. Traitement chirurgical page 29

2.2.2. Traitement adjuvant de radio-chimiothérapie page 30

2.2.2.3. Place des implants imprégnés de Carmustine page 31 
2.4.1.3. Caractéristiques morphologiques propres aux métastases cérébrales

page 48

2.4.2 Séquences fonctionnelles

page 51

2.4.2.1. Imagerie de perfusion

page 52

2.4.2.2. Imagerie de diffusion

page 65

2.4.3 Séquence métabolique : la spectroscopie par résonance magnétique (SRM)

page 69

2.5 Place de la médecine nucléaire dans le suivi des tumeurs cérébrales irradiées

page 75

2.5.1 Généralités page 75

2.5.1.1. La Tomographie par Emission Mono-Photonique (TEMP) page 75

2.5.1.2. La Tomographie par Emission de Positons (TEP) page 75

2.5.2 Radio-pharmaceutiques page 76

2.5.2.1. La TEP au ${ }^{18}$ F-FDG page 76

2.5.2.2. Nouveaux radio-pharmaceutiques utilisés en neuro-oncologie page 78

3- MATERIEL ET METHODES page 84

3.1 Population étudiée page 84 
4.2.2.3. Association des deux techniques d'imagerie de perfusion 
4.2.4. Evaluation de la concordance des résultats de l'interprétation de l'IRM multimodale entre les lecteurs junior et senior page 126

4.3. Evaluation des données relatives à la TEP-TDM à la ${ }^{18}$ F-FDOPA page 128

4.3.1. Performances diagnostiques de la TEP-TDM à la ${ }^{18}$ F-FDOPA par rapport au goldstandard page 128

4.3.2. Evaluation de la concordance des résultats de l'interprétation de l'IRM multimodale et de la TEP-TDM à la ${ }^{18}$ F-FDOPA page 129

5- DISCUSSION page 133

6- CONCLUSION page 149

7- BIBLIOGRAPHIE page 150

8- ANNEXE page 170

9- TABLE DES ILLUSTRATIONS page 171

10- LISTE DES ABREVIATIONS page 175

11- TABLE DES MATIERES page 178

12- SERMENT D'HIPPOCRATE page 184 


\section{2 - SERMENT D'HIPPOCRATE}

En présence des Maîtres de cette école, de mes chers condisciples et devant l'effigie d'Hippocrate, je promets et je jure d'être fidèle aux lois de l'honneur et de la probité dans l'exercice de la médecine.

Je donnerai mes soins gratuits à l'indigent, et n'exigerai jamais un salaire au-dessus de mon travail.

Je ne permettrai pas que des considérations de religion, de nation, de race, viennent s'interposer entre mon devoir et mon patient.

Admis dans l'intérieur des maisons, mes yeux ne verront pas ce qui s'y passe. Ma langue taira les secrets qui me seront confiés, et mon état ne servira pas à corrompre les moeurs, ni à favoriser le crime.

Respectueux et reconnaissant envers mes Maîtres, je rendrai à leurs enfants l'instruction que j'ai reçue de leur père.

Que les hommes m'accordent leur estime si je suis fidèle à mes promesses, que je sois couvert d'opprobre et méprisé de mes confrères si j'y manque. 


\section{RESUME}

Objectifs: Définir les meilleurs outils en IRM multimodale permettant de distinguer radionécrose et récidive tumorale, dans le suivi des tumeurs cérébrales primitives et secondaires irradiées.

Corréler les résultats obtenus en IRM multimodale avec ceux de la TEP-TDM à la ${ }^{18}$ F-FDOPA.

Matériel et méthodes: Cette étude prospective monocentrique, réalisée sur 2 ans, a inclus des patients suspects de récidive tumorale ou de lésions radio-induites, ayant bénéficié d'un protocole standardisé d'IRM multimodale, sur un appareil d'1,5T et d'un examen TEP-TDM à la ${ }^{18}$ F-FDOPA. L'IRM incluait des séquences conventionnelles, fonctionnelles de perfusion T1, T2*et de diffusion et une spectroscopie mono-voxel réalisée en zone suspecte.

Des données IRM morphologiques, fonctionnelles et métaboliques étaient analysées en double lecture, par un radiologue expérimenté et un junior.

Le critère principal de jugement était le diagnostic porté de radionécrose ou de récidive tumorale, lors de l'interprétation de l'IRM multimodale, en comparaison au gold-standard disponible. Le critère secondaire était l'appréciation de la concordance des résultats obtenus entre l'IRM multimodale et la TEP-TDM à la ${ }^{18} \mathrm{~F}$-FDOPA.

Résultats : 61 patients ont été inclus, d'âge moyen égal à 59 ans, dont 35 (57\%) étaient suivis pour tumeurs gliales et 26 (43\%) pour métastases cérébrales.

La concordance entre l'interprétation de I'IRM multimodale et le gold-standard était excellente $(k=0,84)$.

Un rehaussement lésionnel était statistiquement corrélée aux cas de récidive tumorale $(p=0,018)$, sans influence du pattern de rehaussement $(p=1)$.

Des valeurs élevées de $r$ VSC $(p<0,001)$ et $r$ HP ( $p=0,001)$ étaient significativement associées aux cas de progression, avec des taux plus faibles de PSR $(p<0,001)$. Les valeurs de $k_{\text {trans }}$ moyen $(p=0,001), r k_{\text {trans }}(p=0,035), r$ IAUGC $(p<0,001)$ étaient plus élevées en cas de récidive. L'analyse multivariée portant sur l'association des deux techniques de perfusion a montré que $r$ VSC $(p=0,005)$ et $r k_{\text {trans }}(p=0,045)$ étaient déterminants entre les deux groupes.

Les séquences de diffusion et spectroscopiques n'étaient pas discriminantes entre les deux groupes.

$84 \%$ des cas analysés en IRM multimodale et TEP-TDM à la ${ }^{18}$ F-FDOPA étaient concordants $(k=0,65)$.

Conclusion: L'imagerie de perfusion présente les meilleures performances diagnostiques pour distinguer radionécrose et récidive tumorale. Les paramètres $r$ VSC et $r k_{\text {trans, }}$ sont les meilleurs outils pour différentier les deux entités dans notre étude.

La TEP-TDM à la ${ }^{18} \mathrm{~F}$-FDOPA montre une bonne concordance avec les résultats de I'IRM multimodale. Les cas discordants peuvent être en partie expliqués par une surexpression de LAT 1 dans le tissu nécrotique irradié. 